\title{
Flexible and wearable electrochemical biosensors based on two-dimensional materials: Recent developments
}

\author{
Minu Mathew ${ }^{1} \cdot$ Sithara Radhakrishnan ${ }^{1} \cdot$ Antara Vaidyanathan $^{2} \cdot$ Brahmananda Chakraborty $^{3,4}$. \\ Chandra Sekhar Rout ${ }^{1}$ (1)
}

Received: 26 August 2020 / Revised: 3 October 2020 / Accepted: 9 October 2020 / Published online: 23 October 2020

(C) Springer-Verlag GmbH Germany, part of Springer Nature 2020

\begin{abstract}
The research interest in wearable sensors has tremendously increased in recent years. Amid the different biosensors, electrochemical biosensors are unparalleled and ideal for the design and manufacture of such flexible and wearable sensors because of their various benefits, including convenient operation, quick response, portability, and inherent miniaturization. A number of studies on flexible and wearable electrochemical biosensors have been reported in recent years for invasive/non-invasive and real-time monitoring of biologically relevant molecules such as glucose, lactate, dopamine, cortisol, and antigens. To attain this, novel two-dimensional nanomaterials and their hybrids, various substrates, and detection methods have been explored to fabricate flexible conductive platforms that can be used to develop flexible electrochemical biosensors. In particular, there are many advantages associated with the advent of two-dimensional materials, such as light weight, high stretchability, high performance, and excellent biocompatibility, which offer new opportunities to improve the performance of wearable electrochemical sensors. Therefore, it is urgently required to study wearable/flexible electrochemical biosensors based on twodimensional nanomaterials for health care monitoring and clinical analysis. In this review, we described recently reported flexible electrochemical biosensors based on two-dimensional nanomaterials. We classified them into specific groups, including enzymatic/non-enzymatic biosensors and affinity biosensors (immunosensors), recent developments in flexible electrochemical immunosensors based on polymer and plastic substrates to monitor biologically relevant molecules. This review will discuss perspectives on flexible electrochemical biosensors based on two-dimensional materials for the clinical analysis and wearable biosensing devices, as well as the limitations and prospects of the these electrochemical flexible/wearable biosensors.
\end{abstract}

Keywords Biosensor $\cdot 2 \mathrm{D}$ materials $\cdot$ Flexible/wearable sensors $\cdot$ Electrochemical sensors

$\overline{\text { Published in the topical collection 2D Nanomaterials for Electroanalysis }}$ with guest editor Sabine Szunerits.

Minu Mathew and R. Sithara contributed equally to this work.

Brahmananda Chakraborty

brahma@barc.gov.in

$\triangle$ Chandra Sekhar Rout csrout@gmail.com; r.chandrasekhar@jainuniversity.ac.in

1 Centre for Nano and Material Science, Jain University, Jain global campus, Jakkasandra, Ramanagara, Bangalore 562112, India

2 Department of Chemistry, Ramnarain Ruia Autonomous College, Matunga, Mumbai 40085, India

3 High Pressure and Synchrotron Radiation Physics Division, Bhabha Atomic Research Centre, Trombay, Mumbai 40085, India

4 Homi Bhabha National Institute, Mumbai 40094, India

\section{Introduction}

Since the evolution of smartphones, wearable sensors have gained enormous interest, as they can be explored for various applications through connecting and communicating with a smartphone in real time [1]. Specifically, contact between smartphones and wearable devices allows signals obtained from the human body to be tracked and analyzed, and many custom-wearable sensors have been developed for biomedical and sports applications [2]. Flexibility is a major factor in the manufacturing of each part, including batteries and flexible displays that can be used in wearable devices, in order to improve the suitability and sensitivity of wearable devices [3]. The potential of biosensors is immense, considering the various applications for wearable devices, due to the comprehensive feasibility of personalized diagnosis and patient-specific analysis [4]. Specific interactions between sensing probes and target, 
such as antibodies, enzymes, or nucleic acids, are monitored through various methods, including plasmonic, fluorescent, colorimetric, and electrochemical techniques [5-8]. From a biosensor point of view, accurate and early detection of analytes is a major goal that can be attained and enhanced via the design of wearable devices that can be utilized for personalized health care monitoring [9]. According to this view, integrating flexibility to biosensors has attracted more attention due to the basic need for wearable biosensors. Nevertheless, depending on the different biosensors, several problems can be encountered. For example, in the absence of advanced analytical techniques, colorimetric techniques are inadequate for the naked eye monitoring of extremely small concentrations of target molecules [10]. Because of these challenges, selecting appropriate methods for the operation and fabrication of flexible biosensors is needed to apply these technologies to future wearable biosensors. Considering the different types of biosensors, electrochemical biosensors have a significant role to play and fulfill all the requirements for flexible biosensor formation. The electrochemical biosensor monitors the electrochemical signal produced by the reaction between the analyte molecules (target molecules) and the sensing probe [11]. The benefit of electrochemical biosensors is that different electrochemical techniques can be used based on the target molecules and environment such as potentiometry, amperometry, electrochemical impedance spectroscopy, and cyclic voltammetry $[12,13]$. Moreover, these electrochemical biosensors are unparalleled and suited for the design and manufacture of such flexible and wearable sensors due to their various advantages, including convenient operation, fast response, portability, and inherent miniaturization [14]. Besides, conductivity flexibility carries a major role in fabricating flexible electrochemical biosensors. In this context, different materials and methods such as nanomaterials coupled with polymer substrates, printing techniques, or highly conducting polymers have been put forward to manufacture conductive flexible substrates or to design electrochemical platforms on flexible substrates [15-18]. In particular, there are many advantages related to the emergence of twodimensional materials, such as light weight, high stretchability, high efficiency, and excellent biocompatibility, which offer new opportunities to improve the performance of wearable electrochemical sensors. Graphene and its inorganic analogs, such as transition metal dichalcogenides (TMDs), and MXenes have gained huge attention as supporting substrates and transduction elements in a wide range of biosensing applications [19]. What makes layered two-dimensional materials more interesting is their extraordinary properties relative to their bulk form. Two-dimensional materials are ideal candidates for biosensor applications due to the high density of active sites on the surface.
Moreover, the two-dimensional material family can display a wide range of electronic properties, ranging from metallic/semimetallic (e.g., graphene, $\mathrm{TaS}_{2}, \mathrm{VS}_{2}$ ) to semiconducting $\left(\mathrm{MoS}_{2}, \mathrm{WS}_{2}\right.$, black phosphorus) to insulating (e.g., h-BN) [20]. The surface chemistry of two-dimensional materials can be modified via defect engineering or functionalization, these tailored twodimensional materials can selectively respond to specific target molecules with outstanding sensitivity [21-23]. The layer dependent band structure, combined with their various properties, and the capability to synthesize heterostructures (transfer process/direct growth), make two-dimensional materials an emerging class of nanomaterials for upcoming biosensors in future and biomedical applications [24, 25]. Mainly, this review will address some of the latest development in flexible electrochemical sensors based on various twodimensional materials for next-generation electrochemical biosensors and other health care applications. We present a discussion of target biomolecules, working principles, fabrication approaches, and flexible electrochemical biosensors based on two-dimensional materials integrated with stretchable materials and structures. Besides, recent studies on flexible electrochemical biosensors with two-dimensional materials are classified into enzymatic/non-enzymatic biosensors and affinity biosensors (immunosensors), and discussed based on the substrates including polymeric and plastic substrates to detect biologically relevant molecules. This analysis provides an insight into the recent development in research that needs to be used with two-dimensional materials at its core for the realization of next-generation electrochemical biosensors.

\section{Wearable sensor types}

The physiological parameters can be detected through two types of sensors: invasive (in vivo) and non-invasive (in vitro) given in Fig. 1. Invasive sensors (sometimes referred to as intrusive sensors) require body fluids obtained by injecting or incising into the body. Continuous monitoring of the parameters using wearable monitors is of high significance in some cases, such as glucose monitoring in diabetic patients, athletes' fitness monitoring, oxygen saturation monitoring, cholesterol tracking in cardiac patients, and drug efficacy monitoring for various diseases. In such cases, invasive sensors that involve corporal fluids, including blood, serum, etc., are not suitable for measuring and monitoring. Blood contamination is also extremely probable, as needles have been misused [26]. Non-invasive sensors need no human/ animal body fluids collected by the injection or incision to enter the body, making it more enticing and less painful for 
Fig. 1 Classification of wearable biosensors

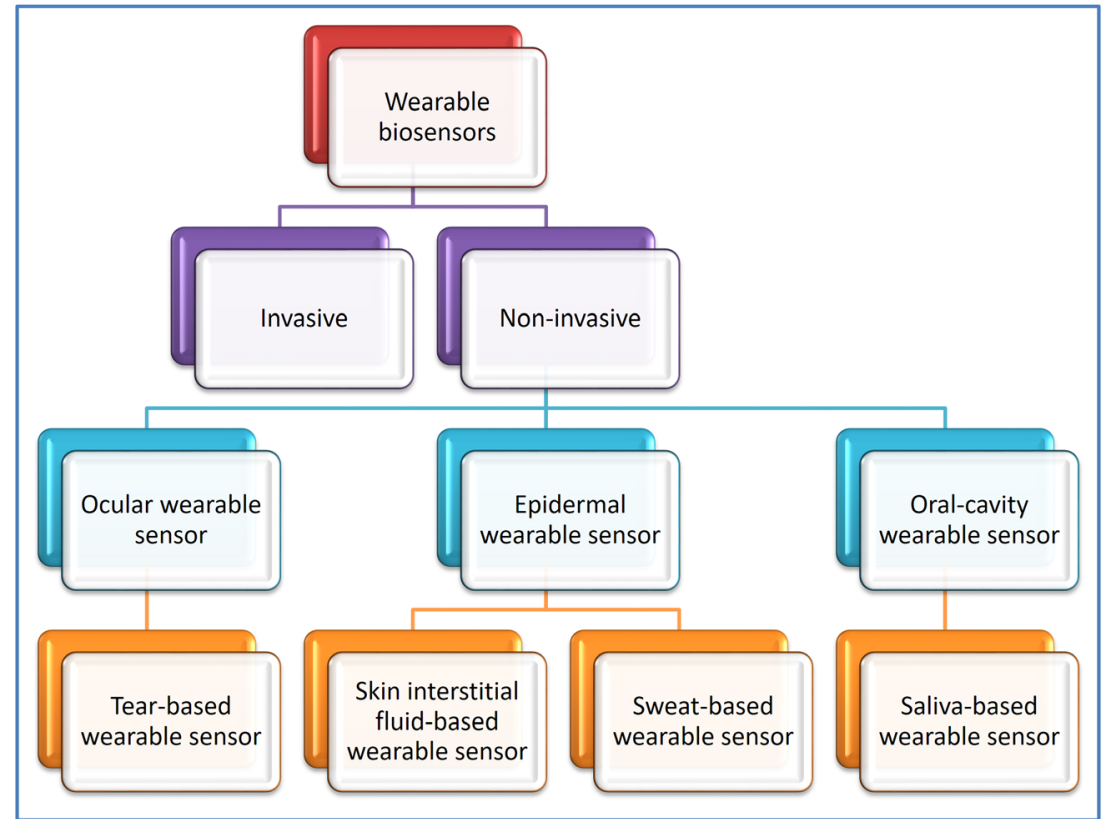

the user. Saliva, sweat, tears, or skin interstitial fluids may be used by non-invasive sensors [26].

\section{Enzymatic and nonenzymatic wearable/flexible bio- sensors based on two-dimensional materials}

\section{Glucose}

Glucose is an essential energy source for cellular function in the human body [27]. Excess blood glucose induces a hyperglycemic condition that causes organ damage, dysfunction, and failure of several organs such as the eyes, renal, nervous, heart, and blood vessels in the long run [28]. Thus, glucose detection in human blood is of medically critical importance in diagnosing diabetes. In 1975, Yellow Spring Instrument (YSI) commercialized the first electrochemical glucose sensors [29]. The advantages of electrochemical biosensors are broad-linear response ranges, low limit of detection, repeatability, and optimal stability, resulting in greater use compared to thermal biosensors [30]. Blood glucose (BG) and perspiration-based glucose levels differ in diabetic patients between $10 \mu \mathrm{M}$ and $0.7 \mathrm{mM}$ [31]. Different electrochemical-glucose sensors have been developed for glucose monitoring, such as enzymatic [32] and non-enzymatic. Based on the mechanism, they are classified as (i) firstgeneration sensors based on natural oxygen substrates and the detection of the emissible hydrogen peroxide shown in the following Eq. 1 [33, 34], (ii) second-generation based on redox mediators [35] such as ferrocene [36] and ferricyanide [37], and (iii) third-generation sensors that are allowing the direct transfer of electrons between the enzyme and the electrode without any natural or synthetic mediators. However, these commonly produced glucose sensors depend on the glucose oxidase (GOx) enzyme; they endure from thermal as well as chemical GOx instability and $\mathrm{GO}_{\mathrm{X}}$ immobilization processes and a complex enzyme. The limitations mentioned above are overcome by non-enzymatic sensors using functional nanomaterials, with a variety of advantages of high stability, low costs, ease of use, and various criteria for its use [38].

glucose $+\mathrm{H}_{2} \mathrm{O}+\mathrm{O}_{2} \stackrel{\mathrm{GOx}}{\rightarrow}$ gluconic acid $+\mathrm{H}_{2} \mathrm{O}_{2}$

\section{Two-dimensional material-based wearable/flexible sensor for the detection of glucose}

The glucose sensors based on graphene can be categorized into two categories: (i) invasive and (ii) non-invasive.

Invasive Zhao et al. prepared a flexible electrode based on a three-dimensional (3D) monolithic nanoporous gold scaffold (NPG) backed by graphene paper (GP), which was further enhanced with the use of an efficient and straightforward ultrasonic electrodeposition method for the extremely compact, well-scattered, and ultrasound binary platinum cobalt ( $\mathrm{PtCo}$ ) alloy nano-particles. This non-enzymatic sensor showed a broad linear range from $35 \mu \mathrm{M}$ to $30 \mathrm{mM}, 5 \mu \mathrm{M}$ limit of detection (LOD), and $7.84 \mu \mathrm{A} \mathrm{cm}^{-2} \mathrm{mM}^{-1}$. The proposed roughness factor and larger surface area of this prepared electrode greatly improved the electrochemical response of glucose oxidation and reduced the intervention of substances, including uric acid, dopamine, and ascorbic acid resulting in increased sensitivity and selectivity for glucose detection. This roughness factor is stated as the ratio of active surface area 
over the electrode, and this NPG/PtCo/GP electrode showing a high roughness factor is responsible for large faradic currents for glucose oxidation and thus responsible for its excellent electrochemical sensing. This flexible $\mathrm{PtCo} / \mathrm{NPG} / \mathrm{GP}$ (Fig. 2a) electrode with excellent mechanical bending strength allows it to be integrated into an implantable or a wearable biomedical device. This flexible sensor ensures high selectivity, reproducibility, and stability, but has high production costs due to the different materials used [39]. Another similar research is recorded, proposing a non-enzymatic system based on the graphene paper by $\mathrm{He}$ et al. [40]. In this work, the electrode is prepared by printing a $3 \mathrm{D}$ porous graphene carbon nanotube (CNT) assembly on a two-dimensional graphene paper using ionic liquid (IL) as a binder (provides good adhesion between two-dimensional graphene paper and 3D porous graphene/CNT). Bimetallic platinum gold (PtAu) alloy nanoparticles were deposited on this freestanding composite by the ultrasonic-electrochemical method. The step involved in the fabrication of this sensor is shown in Fig. 2b. This bimetallic nanoparticle shows increased electrochemical activity and the ability to reduce the surface poisoning by two mechanisms for glucose oxidation, (i) promoted mechanism and (ii) effect of electronic charge transfer, resulting in increased glucose catalytic activity. This sensor shows a linear range from 0.1 to

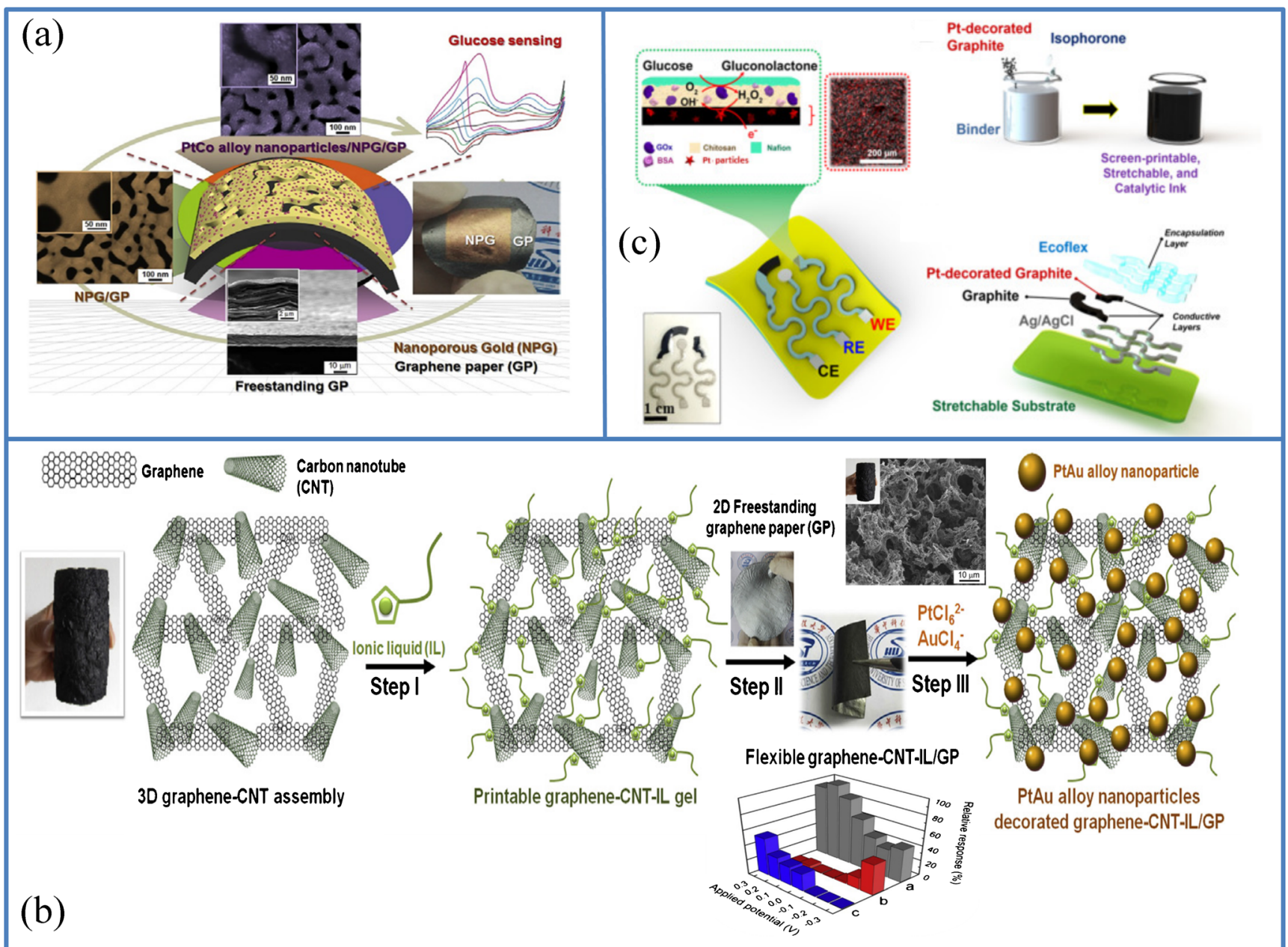

Fig. 2 Different kinds of glucose sensors based on two-dimensional materials. a Freestanding, flexible PtCo/NPG/GP electrode nonenzymatic invasive glucose biosensor [Source: reprinted from Anshun Zhao, Zhaowei Zhang, Penghui Zhang, Shuang Xiao, Lu Wang, Yue Dong, Hao Yuan, Peiwu Li, Yimin Sun, Xueliang Jiang, Fei Xiao, 3D nanoporous gold scaffold supported on graphene paper: freestanding and flexible electrode with high loading of ultrafine PtCo alloy nanoparticles for electrochemical glucose sensing, Analytica Chimica Acta, 938 (2016) 63-71,, with permission from Elsevier]. b Steps involved in the preparation of PtAu/graphene-CNT-IL/GP invasive glucose sensor developed by $\mathrm{He}$ et al. with inset figure amperometric response of PtAu/rGOCNT-IL/GP compared with other electrodes and SEM image (source: reprinted from Wenshan He, Yimin Sun, Jiangbo Xi, Abduraouf Alamer Mohamed Abdurhman,Jinghua Ren, Hongwei Duan, Printing graphenecarbon nanotube-ionic liquid gel on graphene paper: towards flexible electrodes with efficient loading of PtAu alloy nanoparticles for electrochemical sensing of blood glucose, Analytica Chimica Acta, 903 (2016) 61-68, with permission from Elsevier). c Diagram showing the design of Pt-decorated graphite stretchable sensor developed by Abellan et al. (source: reprinted from A. Abellán-Llobregat,Itthipon Jeerapan,A. Bandodkar,L. Vidal,A. Canals,J. Wang,E. Morallón- A stretchable and screen-printed electrochemical sensor for glucose determination in human perspiration, Biosensors and Bioelectronics, 91 (2017) 7, with permission from Elsevier) 
$11.6 \mathrm{mM}$ with a LOD of $8.0 \mu \mathrm{M}$ and sensitivity around $0.19 \mathrm{~mA} \mathrm{~cm}^{-2} \mathrm{mM}^{-1}$ and has less sensitivity, but the method of preparation is more straightforward [40].

Non-invasive Lee et al. fabricated a chemical vapor deposition (CVD) grown gold-dried graphene biosensor, using $\mathrm{Ag} / \mathrm{AgCl}$ as a solid-state counterpart electrode that significantly enhances the electrical activity and can detect levels of glucose, even at low concentrations [41]. Here, the gold-doped graphene, as well as the gold mesh, enhanced the electrochemical response over the bare graphene and created a wearable sweat-focused patch for diabetes tracking and feedback therapy. This extendable tool containing a serpentine bilayer of gold mesh and gold-doped graphene provides a secure transmission system for electrical signals. This patch also consists of temperature, glucose, $\mathrm{pH}$ and humidity sensors, and heatactivated microneedles for transcutaneous drug supply. This sector is advanced by miniaturizing the biosensor and creating ultra-thin stretchable equipment to make access to sweat more comfortable [41]. Very recently, Abellan et al. reported (Fig. 2c) a very stretchable and cost-effective non-enzymatic and enzymatic glucose sensor based on platinum graphite [42]. This sensor is produced using screen printing techniques on the polyurethane (PU) substrate, and GOx was immobilized with chitosan and phosphate buffer solution (PBS) used as an enzyme stabilizer. The linear range of sensitivities was recorded from $0 \mathrm{mM}$ to $0.9 \mathrm{Mm}$. This device can extend to $75 \%$ of its original length. This enzymatic device (using GOx), however, achieves superior output compared with the non-enzymatic device, thus placing the strain on the sensor's cycle time. The enzymatic detection improves sensibility and LOD considerably compared to direct oxidation of glucose with the electrode Pt-graphite. The GOx immobilization efficiency of the double reciprocal plot could be found to be correlated with the Lineweaver-Burk equation given below [42].

$I / I_{\mathrm{SS}}=I / I_{\max }+K_{\mathrm{m}}{ }^{\text {app }} / I_{\max } C_{\text {glucose }}$

Here, the steady-state current reached after the addition of glucose is indicated as $I_{\mathrm{SS}}$, the concentration of glucose in bulk as $C_{\text {glucose, }}$ and the maximum current observed at glucose saturation as $I_{\max }$. The apparent Michaelis $\left(K_{\mathrm{m}}{ }^{\mathrm{app}}\right)$ constant was calculated to be around $1.2 \mathrm{mM}$. This result showed that the $K_{\mathrm{m}}{ }^{\text {app }}$ low electrode value indicates its strong affinity to the glucose substrate [42]. Zhihua et al. documented an electrochemical sensor that can detect glucose levels in the interstitial fluid (ISF). Here, the modified graphene was printed onto the working electrode and electrodeposited gold nanoparticles above graphene to improve the efficiency of the sensor via electrochemical polymerization glucose oxidase was immobilized above the working electrode surface for glucose-sensitive detection. Here, hydrogen peroxide $\left(\mathrm{H}_{2} \mathrm{O}_{2}\right)$ can be directly characterized without immobilized GOx and the electrochemical reaction is given below [43].

$$
\begin{aligned}
& \text { GOx }_{\mathrm{ax}}+\text { glucose }+\mathrm{H}_{2} \mathrm{O} \rightarrow \mathrm{GOx}_{\mathrm{red}}+\text { gluconic acid } \\
& \quad+\mathrm{H}_{2} \mathrm{O}_{2} \\
& \mathrm{GOx}_{\mathrm{red}} \rightarrow \mathrm{GOx}_{0 \mathrm{x}}+\mathrm{ne}^{-} \\
& \mathrm{H}_{2} \mathrm{O}_{2} \rightarrow \mathrm{O}_{2}+2 \mathrm{H}^{+}+2 \mathrm{e}^{-}
\end{aligned}
$$

To produce a compact and usable device with a linear range of $0 \mathrm{mM}-2.22 \mathrm{mM}, 16.6-\mu \mathrm{m}$ LOD, the resulting glucose sensor is embedded with the ISF extraction chip. However, as glucose content in the ISF remains well below that of the blood test, the accuracy of reading glucose using the ISF remains under discussion [43]. Recently, many kinds of infrared (IR) lasers have also been implemented for the fast and easy processing of graphene-based materials [44]. These IR lasers dramatically break, recombine, and release $\mathrm{C}-\mathrm{O}, \mathrm{N}-\mathrm{C}$, and $\mathrm{C}=\mathrm{O}$ bonds in polyimides in the form of gases, generating porous graphene nanosheets with structures made up of the heptagon and hexagon lattice structures of more than $85 \%$ carbon. For the first time, Zhang et al. used a substrateassisted electroless deposition (SAED) method for developing a copper nanoparticles-laser-induced graphene (Cu NPs-LIG) enzyme-free glucose biosensor where $\mathrm{Cu}$ nanoparticles are anchored to LIG. The linear range is from $1 \mu \mathrm{M}$ to $6.0 \mathrm{mM}$, with a LOD of $0.39 \mu \mathrm{M}$ and a sensitivity of $495 \mu \mathrm{A} \mathrm{mM}^{-1} \mathrm{~cm}^{-2}$. Electron transfers occur after redox reactions among $\mathrm{Cu}$ NPs and glucose molecules, while the $\mathrm{Cu} /$ graphene system can accelerate the transfer of electron and allow excellent glucose-sensing efficiency. This sensor amperometric response for the determination of glucose is shown in Fig. 3a. Here, the continuous addition of glucose results in a current response in a stepwise manner. This sensor's response is speedy and it takes just $0.49 \mathrm{~s}$ to achieve a steady-state current. The enhanced surface area of this composite contributes to the rapid diffusion of glucose molecules [45]. Later, Hyosang et al. proposed a surface modification method for this LIG using acetic acid and thereby increased the conductivity and sheet resistance of LIG. Pt nanoparticles were then electrodeposited on this acid-modified LIG electrode to fabricate a sweat-based glucose sensor and immobilized GOx by chitosan. The current response of this sensor to a rise in the concentration of glucose was examined amperometrically. It has been shown that the amperometric response of glucose oxidation through linear regression analysis is the direct function $y=a+b^{*} x$, where $a$ is the slope and $b$ is the linear intercept. This established glucose sensor possesses a very linear behavior even in low glucose concentration, and LOD was measured approximately $300 \mathrm{nM}$ by the following equation, which shows its excellent performance compared to $\mathrm{Cu}$ NPsLIG sensors. By testing blood glucose levels with 


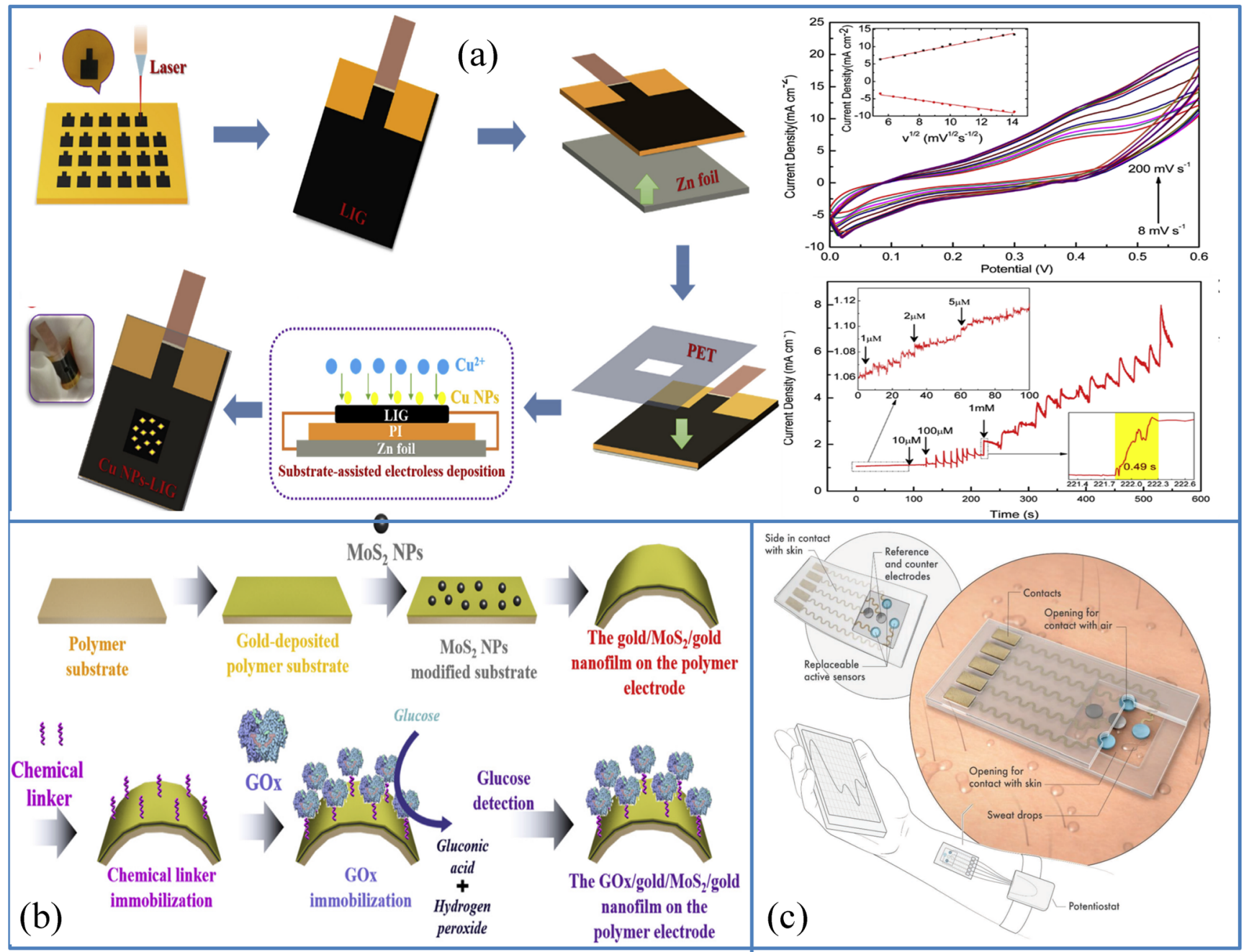

Fig. 3 Different kinds of glucose sensors based on two-dimensional materials. a Fabrication process of $\mathrm{Cu}$ NPs-LIG composite with $\mathrm{CV}$ curves and the amperometric response of Cu NPs-LIG composite (source: reprinted from Yue Zhang,Na Li, Yangjun Xiang, Debo Wang, Peng Zhang, Yanyan Wang, Shan Lu, Rongqing Xu, Jiang Zhao, A flexible non-enzymatic glucose sensor based on copper nanoparticles anchored on laser-induced graphene, Carbon (2020) 506-513), with permission from Elsevier). b Schematic diagram of the manufacture of GOx/gold/ $\mathrm{MoS}_{2} /$ gold nanofilm on the polymer electrode (source: reprinted from Jinho Yoona, Sang Nam Leeb, Min Kyu Shina, Hyun-Woong Kima, Hye

amperometry before and after meals, they showed the market potential of this sweat-based sensor [46].

$\operatorname{LOD}\left(\frac{S}{N}\right.$ ratio $)=3=\frac{\text { average level }_{\text {after stabilization of reaction }}-\text { average level }_{\text {before glucose injection }}}{3 * \text { double standard deviation }}$

Cao et al. fabricated an electrochemical glucose sensor based on 3D paper microfluid that could prevent the interaction between the immobilization of biomolecules and the modification of electrodes. Here, the aldehydefunctionalized counter and reference electrode hydrophilic
Kyu Choia, Taek Leec, Jeong-Woo Choi- Flexible electrochemical glucose biosensor based on GOx/gold/MoS2/gold nanofilm on the polymer electrode, Biosensors and Bioelectronics, 140 (2019) 111343, with permission from Elsevier). $\mathbf{c}$ Diagrammatic illustration of $\mathrm{Ti}_{3} \mathrm{C}_{2} \mathrm{~T}_{\mathrm{x}} / \mathrm{PB}$ based glucose/lactate and $\mathrm{pH}$ sensor developed by Lei et al. (Source: reprinted from Yongjiu Lei, Wenli Zhao, Yizhou Zhang, Qiu Jiang, Jr-Hau He, Antje J. Baeumner, Otto S. Wolfbeis, Zhong Lin Wang, Khaled N. Salama, and Husam N. Alshareef- A MXene-based wearable biosensor systemfor high-performance in vitro perspiration analysis, Small 2019, 1,901,190, with permission from Elsevier)

zone were designed for glucose oxidase immobilization while reduced graphene oxide-tetraethylenepentamine/Prussian blue (rGO-TEPA/PB) modified paper was used as a working electrode to detect and quantify $\mathrm{H}_{2} \mathrm{O}_{2}$ prepared by depositing Prussian blue over rGO-tetraethylenepentamine. The suggested biosensor may be used to quantify glucose over a wide linear range of $0.1 \sim 25 \mathrm{mM}$ under optimum conditions and with a LOD of $25 \mu \mathrm{M}$ [47]. Xuan et al. reports a patchbased flexible electrochemical glucose sensor that was successfully micro-fabricated and patterned on a flexible polyimide (PI) substrate; high-quality graphene oxide (rGO) composite working electrode was used. The electrodes are modeled 
with a technique of photolithography. The working electrode is made from gold- and platinum-deposited rGO before the immobilization of GOx with chitosan. The detection range is from 0 to $2.4 \mathrm{mM}$ and sensitivity around $48 \mu \mathrm{A} \mathrm{mM} \mathrm{cm}^{-1}$; linearity is high at 0.99 and response time is fast at $12 \mathrm{~s} \mathrm{[48].}$

TMDs also show interesting properties for glucose oxidation. Both enzymatic and non-enzymatic methods are repeatedly used for the construction of TMD-based glucose sensors. Wang et al. developed a composite that is used to detect glucose and lactate with $\mathrm{MoS}_{2}$ graphene. It demonstrated rapid response, good selectivity, super reproductiveness, and flexibility, making it possible to apply the promising material in glucose and human sweat lactate monitoring. This composite showed linear ranges for glucose between 5 and $1775 \mu \mathrm{M}$ and lactate between 0.01 and $18.4 \mathrm{mM}$, with respective detection limits of $500 \mathrm{nM}$ and $0.1 \mu \mathrm{M}$ [49].

Yoona et al. reported a $\mathrm{MoS}_{2} / \mathrm{AuNPs} / \mathrm{GOx}$ hybrid bioelectrode (Fig. 3b). This sensor showed an increased electronic signal with an average reduction peak current of $389.9 \mu \mathrm{A}$ compared to Au-deposited GOx polymer electrode $(125.8 \mu \mathrm{A})$. This sensor improved amperometric glucose sensing efficiency with a LOD of $10 \mathrm{nM}$, which suggested an increased sensitivity. This biological sensor is more flexible than the traditional Au-coated silicon electrode $(0.09 \mathrm{~mm})$, where this flexible polymer electrode has a flexural extension of about $3.48 \mathrm{~nm}$ [50].

$\mathrm{NiSe}_{2}$ has a prominent role among TMDs, as it shows increased electrochemical activity due to the active site and two metals' synergistic effects. Besides this, it also shows better efficiency in electron transfer, making it a promising candidate as a biosensor. Vishnu et al. used a simple hydrothermal approach for direct $\mathrm{NiSe}_{2}$ growth on cellulose paper, which is an effective method for developing a disposable glucose sensor. The redox reaction is given below.

$\mathrm{Nise}^{2+} \mathrm{OH}^{-} \leftrightarrow \mathrm{NiseOH}+\mathrm{Se}+\mathrm{e}^{-}$

The following are the mechanisms of electrochemical oxidations of glucose:

$\mathrm{Ni}^{4+}+$ glucose $\rightarrow \mathrm{Ni}^{3+}+$ gluconolactone

$\mathrm{Se}^{3+}+$ glucose $\rightarrow \mathrm{Se}^{2+}+$ gluconolactone

This sensor had excellent sensitivity $\left(0.25 \mathrm{AM}^{-1} \mathrm{CM}^{-2}\right)$ to detect glucose at the potential of electrodes $\left(E_{\mathrm{pp}}=0.55 \mathrm{~V}\right.$ versus $\mathrm{Ag} / \mathrm{AgCl})$ and a low detection limit $(24.8 \mu \mathrm{M})$. This sensor is suitable for real-time applications as it successfully detects glucose in biological fluids such as serum blood and urine [51]. Liu and colleagues produced a flower-like twodimensional copper cobaltite $\left(\mathrm{CuCo}_{2} \mathrm{O}_{4}\right)$ nanosheet anchored on a flexible graphite paper by an easy hydrothermal method following a post-annealing treatment. Due to the transport of electrons with relatively low activation energy between different transition metal cations, spinel cobaltites have higher electrical conductivity and electrochemical properties than monometallic oxides, thus enhancing storage efficiency at a high rate. Graphite paper can also have extra active sites, and electrode manufacturing is streamlined with the benefits of low cost, durability, high mechanical strength, and light weight desirable for flexible electrodes. These functionalized graphite papers can be used as electrodes for non-enzymatic glucose sensors with high selectivity, sensitivity, exceptionally linear response, and low LODs of $5 \mu \mathrm{M}$. Furthermore, those electrodes have excellent supercapacitative properties (1131 $\mathrm{F} \mathrm{g}^{-1}$ at $1 \mathrm{AG}^{-1}$, superior high performance and excellent long-term cycling stability), which have demonstrated great success as a multifunctional platform for wearable biomedical devices [52].

\section{Lactate}

Lactate is the primary anaerobic metabolite. Accumulated lactic acid levels result in lactic acidosis without adequate liver and kidney clearance [53]. Thus, lactic acid in the blood is detected for clinical hypoxia therapy, lactic acidosis, and other acute heart disorders, as well as drug toxicity studies [33, 54]. The most widely used biological recognition factor in the manufacture of L-lactate biosensors is L-lactate dehydrogenase (LDH) and L-lactate oxidase (LOD) [55].

Two-dimensional material based wearable/flexible sensor for detection of lactate For the first time in 2013, Labroo et al. presented a flexible bio-nano sensor based on graphene for lactate detection. It sensitively detected lactate in a quick, steady-state $2 \mathrm{~s}$, from 0.08 to $20 \mathrm{mM}$. Yet, the main drawback of the sensor is that the bending angle and the number of bending replicates increase, while the sensor response decreased [56]. These drawbacks may be due to the macroscopic aggregation or re-stacking of graphene, which may reduce the accessible surface area and reduce the efficiency of the sensor [57]. Lin et al. recently showed a lower concentration sweat lactate sensor that operates on passively expressed eccrine sweat without redox molecules. They developed the mechanism for lactate detection by creating charged ions in the enzyme-substrate through LOD catalyzed reactions, resulting in the efficient modification of the capacitance and resistive behaviors and monitoring by electrochemical impedance spectroscopy (EIS). Here, graphene oxide (GO) nanosheets are dispersed through the pores of a polyamide (PA) membrane, where these elements function as a transducer with an affinity-based detector mechanism shown in Fig. 4b. 1Pyrenebutyric acid-N-hydroxysuccinimide ester 4 (PANHS) linker was successfully used for glutamic acid dehydrogenase and glucose oxidase immobilization. With a detection limit of $1 \mathrm{mM}$, the sensor revealed a dynamic range in both synthetic sweat and human sweat that spiked with the lactate dose [58]. 


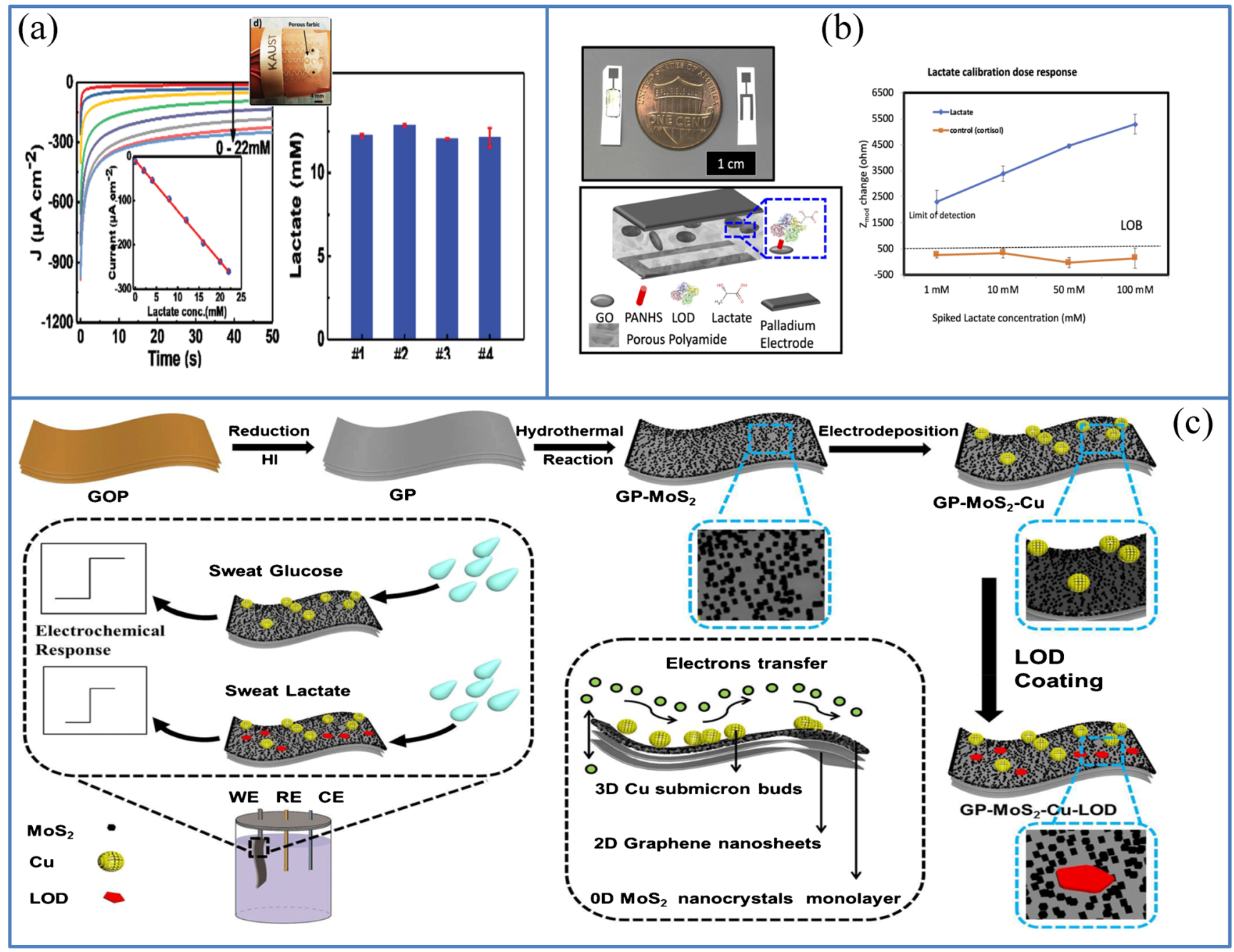

Fig. 4 Lactate-sensing mechanism and sensors based on twodimensional materials. a $\mathrm{Ti}_{3} \mathrm{C}_{2} \mathrm{~T}_{x} / \mathrm{PB}$-based glucose/lactate and $\mathrm{pH}$ sensor and its chronoamperometric response towards lactate sensing (source: reprinted from Yongjiu Lei, Wenli Zhao, Yizhou Zhang, Qiu Jiang, JrHau He, Antje J. Baeumner, Otto S. Wolfbeis, Zhong Lin Wang, Khaled N. Salama, and Husam N. Alshareef- A MXene-based wearable biosensor system for high-performance in vitro perspiration analysis, Small 2019, 1,901,190, with permission from Elsevier). b Schematic representation of GO nanosheets embedded between the references and working electrode of the polyamide membrane. Lactate calibration dose-response in human

Wang et al. developed a new category of sweat sensors made by depositing a $\mathrm{Cu}$ submicron bud with a monolayer of $\mathrm{MoS}_{2}$ nanocrystals, on stand-alone graphene paper (GP) for biofunctional glucose and lactate detection given in Fig. 4c. Here, $\mathrm{Cu}$ submicron buds are well packed in a short time with an easy process for the immobilization of the enzymes and the rapid transport of electrons. The sensor exhibited a linear range from 5 to $1775 \mu \mathrm{M}$, with LOD of $500 \mathrm{nM}$ and a sensitivity of about $3.38 \mathrm{mAcm}^{-2} \mathrm{mM}^{-1}$. The positive interactions between highly loaded 3D Cu-submicron buds on graphene provided more active sites for electrochemical reactions and improved the electrocatalytic efficiency of this sensor through sweat (source: reprinted from Kai-Chun Lin, Sriram Muthukumar, Shalini Prasad, Flex-GO (Flexible graphene oxide) sensor for electrochemical monitoring lactate in low-volume passive perspired human sweat, Talanta (2020), with permission from Elsevier). (c) Schematic representation of fabrication of $\mathrm{GP}-\mathrm{MoS}_{2}-\mathrm{Cu}-\mathrm{LOD}$ electrode (source: reprinted from Zhengyun Wang, Shuang Dong, Mengxi Gui, Muhammad Asif, Wei Wang, Feng Wang, Hongfang Liu, Graphene paper supported $\mathrm{MoS}_{2}$ nanocrystals monolayer with Cu submicron-buds: high-performance flexible platform for sensing in sweat, Analytical Biochemistry (2018) 82-89, with permission from Elsevier)

interfacial contact between graphene and monolayer $\mathrm{MoS}_{2}$. The apparent $K_{\mathrm{M}}$ value as per Eq. 6 was around $10.1 \mathrm{mM}$, which is less than the reference value $(16.7 \mathrm{mM})$, and this shows a high affinity for lactate in the modified electrode [49]. The first approach for the detection of glucose and lactate using MXene was fabricated by Lei et al. and compared to carbon nanotube (CNT)/Prussian blue (PB) and graphene/PB composites for $\mathrm{H}_{2} \mathrm{O}_{2}$ detection to overcome these hurdles. $\mathrm{Ti}_{3} \mathrm{C}_{2} \mathrm{~T}_{x} / \mathrm{PB}$ composites, as in Fig. $3 \mathrm{c}$ and Fig. $4 \mathrm{a}$, showed enhanced electrochemical performance due to their excellent conductivity and electrochemical operation. All three active sensors stand independently and can be replaced at any time, 
which is one of the outstanding properties of this biosensor. This biosensor's revolutionary architecture makes adequate $\mathrm{O}_{2}$ provision during the analysis. The working electrode was prepared on porous and ultrathin electrodes by immobilizing with GOx for glucose and lactate oxidase (LO) sensing. Electrodes were then mounted in the sensor system; $\mathrm{Ag} /$ $\mathrm{AgCl}$ inks and platinum films act as reference and counter electrodes, respectively. The pH sensor's working electrode consists of polyaniline (PANI), and the reference electrode is of $\mathrm{Ag} / \mathrm{AgCl}$. The glucose oxidase-modified electrode showed a linear amperometric response to glucose in artificial sweat, with a LOD of $0.33 \times 10^{-6} \mathrm{M}$ and a sensitivity of $35.3 \mu \mathrm{A} \mathrm{mM}^{-1} \mathrm{~m}^{2}$. Similarly, modified lactate oxidase electrodes had a linear relation to lactate rates ranging from $10 \times$ $10^{-6}$ to $22 \times 10^{-3} \mathrm{M}$, with a LOD of $0.67 \times 10^{-6} \mathrm{M}$ with a sensitivity of $11.4 \mu \mathrm{A} \mathrm{mM} \mathrm{m}^{-1} \mathrm{~m}^{2}$ determined by chronoamperometry shown in Fig. 4a, which also shows the calibration plot of the corresponding sensor. This biosensor is used to obtain sufficiently sweat and to calculate glucose, lactate, and $\mathrm{pH}$ values independently in less than $2 \mathrm{~min}$, respectively [59].

\section{Biologically relevant molecules}

Small molecules, including adenine dynucleotides (NADH), hydrogen peroxide $\left(\mathrm{H}_{2} \mathrm{O}_{2}\right)$, nicotinamide, adenosine triphosphate (ATP), and neurotransmitters, play essential roles in living organisms. These small molecules' abnormal levels frequently result in physiological disorders and illnesses. Hence, it is necessary to recognize these small in vivo and in vitro molecules [60]. Neurotransmitters are a variety of physiologically crucial compounds in the living body's metabolic system, including ascorbic acid (AA) [61], dopamine (DA, 26-40 $\mathrm{nmol} \mathrm{L}^{-1}$ ) [62], serotonin, uric acid (UA) [63], NADH, and so on. They are essential in human metabolism as they affect the co-existence between the central nervous system and the serum.

The electrochemical intervention of AA and its high concentrations is a significant problem in the biological determination of UA [64]. The following reaction reveals uricase enzyme procedures based on $\mathrm{H}_{2} \mathrm{O}_{2}$ amperometric detection [33]:

Uric acid $+\mathrm{O}_{2}+2 \mathrm{H}_{2} \mathrm{O} \rightarrow$ allantoin $+\mathrm{H}_{2} \mathrm{O}_{2}+\mathrm{CO}_{2}$

Other enzymatic methods rely on the electron transfer mediators and the reactions are as follows [33]:

$$
\begin{aligned}
& \text { Uric acid }+ \text { mediator }_{(\mathrm{ox})}+2 \mathrm{H}_{2} \mathrm{O} \stackrel{\text { uricase }}{\rightarrow} \text { allantoin } \\
& + \text { mediator }_{(\mathrm{red})}+\mathrm{CO}_{2}+2 \mathrm{H}^{+} \\
& \text {mediator }_{(\mathrm{red})} \rightarrow \text { mediator }_{(\mathrm{ox})}+2 \mathrm{e}^{-}
\end{aligned}
$$

Hydrogen peroxide analyte plays a major role in biochemical pathways, and a large number of researchers have been drawn into the production of a biosensor to detect its level [60, 65]. $\mathrm{H}_{2} \mathrm{O}_{2}$ is identified as a possible biomarker for many forms of cancer cell types, and it is critical to creating a responsive and accurate method to reliably detect $\mathrm{H}_{2} \mathrm{O}_{2}$ concentration in physiological conditions for early cancer screening and diagnosis [66]. The electrochemical detection of $\mathrm{H}_{2} \mathrm{O}_{2}$ can be performed either with enzymatic $[67,68]$ or non-enzymatic approaches $[65,68]$ similar to glucose sensing approaches $[65$, $68]$. This section gives a detailed explanation of the biosensors based on two-dimensional materials to detect these biologically relevant molecules [69].

Flexible electrochemical sensors based on two-dimensional materials for the detection of biologically relevant molecules Flexible fiber made of graphene was an excellent choice as a biosensor (i) due to its conductivity; (ii) because the flexible and robust nature of the fibers allow it to be used in electrochemical tests directly as a working electrode; and (iii) because it could be further functionalized by chemical deposition to enhance the efficiency. For the first time, using these properties, Cai and his group reported a flexible biosensor (Fig. 5a) based on GFfunctionalized $\mathrm{NiCO}_{2} \mathrm{O}_{4}$ nanowires $\left(\mathrm{GF} / \mathrm{NiCo}_{2} \mathrm{O}_{4}\right)$. This sensor showed a detection limit of $50 \mu \mathrm{M}, 0.2 \mu \mathrm{M}$, and $0.1 \mu \mathrm{M}$ for $\mathrm{AA}, \mathrm{UA}$, and $\mathrm{DA}$, respectively [70]. This gives rise to the idea of using flexible fiber-based sensors for wearable applications.

Molybdenum disulfide $\left(\mathrm{MoS}_{2}\right)$ is considered to be a suitable two-dimensional material for electrochemical sensing applications among transition metal dichalcogenides (TMDs) due to the high mobility of electrons and exposed active sites, bandgap characteristics, and higher conductivity [71]. Rinky et al. first documented the use of $\mathrm{MoS}_{2}$ for a flexible and low-cost UA electronic sensor, while $\mathrm{MoS}_{2}$ was developed using hydrothermal synthesis directly on an aluminum foil. Their method of synthesis shown in Fig. $5 \mathrm{~b}$ resulted in the formation of higher 1T phase content in $\mathrm{MoS}_{2}$, increasing its sensitivity. This synthesized $\mathrm{MoS}_{2}$ micro-flower contains a large number of interconnected nanosheets, which improved the $\mathrm{MoS}_{2}$ surface area, making it simple for the electrolyte to penetrate the electrode as well as the oxidation reaction of ions. This sensor showed a detection limit of $1.169 \mu \mathrm{M}$, quick response time, excellent selectivity, reproducibility, and sensitivity of $98.3 \pm$ $1 \mathrm{nA} \mu \mathrm{M}^{-1}$ in a dynamic range between 10 and $400 \mu \mathrm{A} \mathrm{mM}^{-1}$. Cyclic voltammetry and amperometry were investigated for the electrochemical characteristics of folic acid (FA) on this prepared electrode, where this flexible paper sensor showed a sensitivity of approximately $229.07 \mu \mathrm{A} \mathrm{mM}^{-1} \mathrm{~cm}^{-2}$ and a detection limit of nearly $3.68310^{-8} \mathrm{M}$ [72]. 


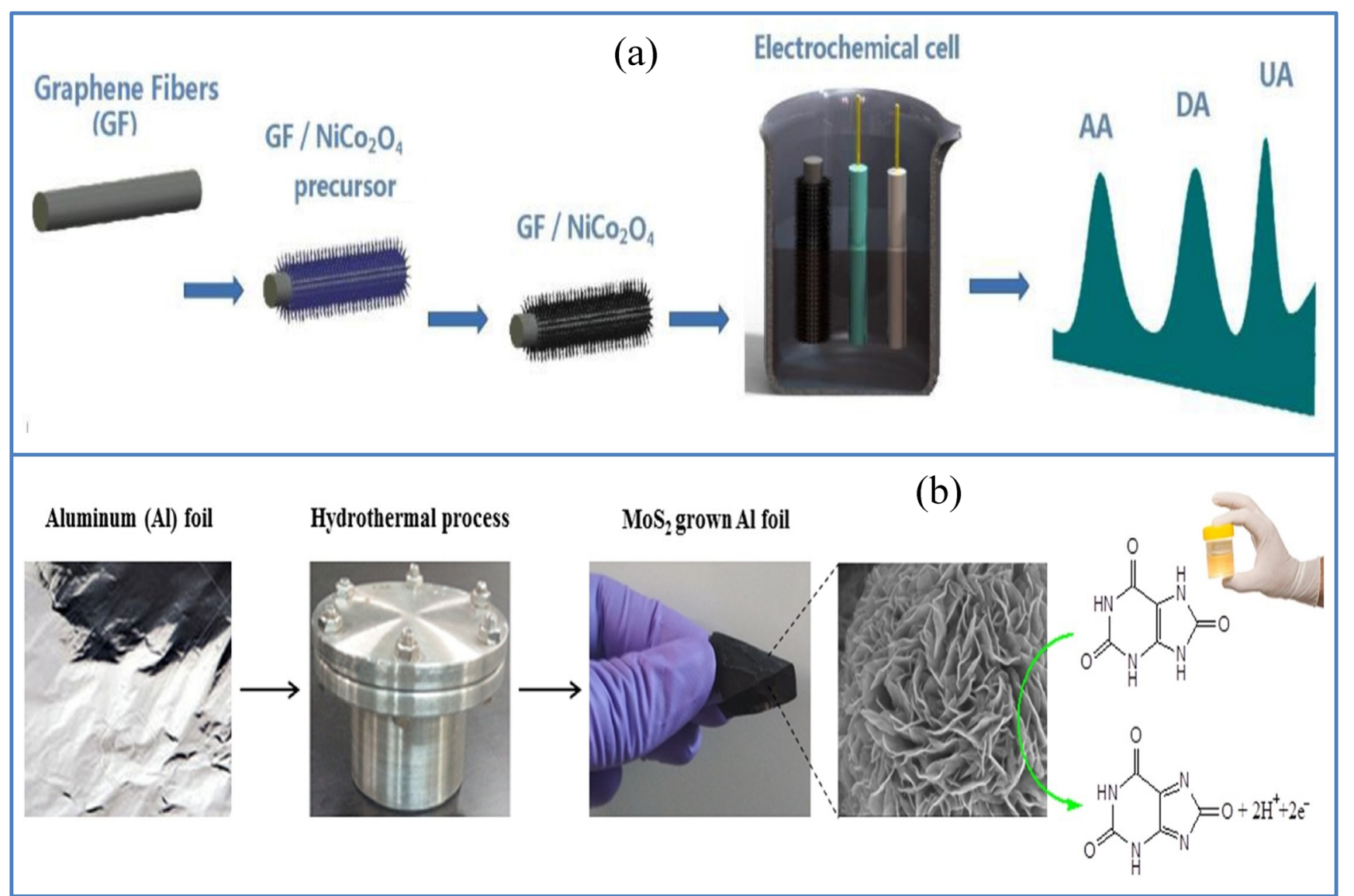

Fig. 5 Flexible as well as a wearable sensor for detection of small molecules like ascorbic acid, uric acid, and so on. a Fiber-based GF/ $\mathrm{NiCo}_{2} \mathrm{O}_{4}$ sensor for the detection of AA, DA, and UA (source: reprinted from Weihua Cai, Jianwei Lai, Ting Lai, Haoting Xie, Jianshan YeControlled functionalization of flexible graphene fibers for the simultaneous determination of ascorbic acid, dopamine and uric acid, Sensors and Actuators B: Chemical, 224 (2016)225-232, with permission from

A three-electrode laser scribed graphene (LSG) structure on cheap paperboards shown in Fig. 6a (silver ink is coated to enhance electrochemical stability) has been demonstrated by Araujo et al. The price for every unit is only US\$ 0.025 (including paper board, $\mathrm{CO}_{2}$ laser tube, and silver ink), and all caffeic acid, ascorbic acid, and picric acid can be analyzed using this sensor [73]. Nayak et al. also produced a modified Pt three-electrode laser scribed graphene structure on the PI (Fig. 6b). This sensor is capable of detecting biomarkers such as UA, AA, and DA, and with a high sensitivity of $250.69 \mu \mathrm{A} \mathrm{Mm}^{-1} \mathrm{~cm}^{-2}, 6995.6 \mu \mathrm{A} \mathrm{Mm}^{-1} \mathrm{~cm}^{-2}$, and $8289 \mu \mathrm{A} \mathrm{Mm}^{-1} \mathrm{~cm}^{-2}$ at a wide range of concentrations [74]. A different approach, i.e., 3D printing-fused deposition modeling (FDM), was used by Rafel et al. recently for the manufacture of a biosensor to track nitric acid and uric acid from saliva and urine, as well as for blood plasma glucose sensing. Here, the carboxylic acid oxygenate group present with 3D-printed G-PLA provides an ideal condition for the immobilization of the enzyme by cross-linking glutaraldehyde
Elsevier). b Schematic fabrication procedure for preparation of $\mathrm{MoS}_{2}$ grown on Al foil for detection of UA. (Source: reprinted from Rinky Sha,Nandimalla Vishnu, Sushmee Badhulika-MoS 2 based ultra-low-cost, flexible, non-enzymatic and non-invasive electrochemical sensor for highly selective detection of uric acid in human urine samples, Sensors and Actuators B: Chemical, 279 (2019) 53-60, with permission from Elsevier)

to a plasma glucose sensor construction. The combined mechanical polishing and solvent immersion treatment enhance the electrochemical performance of the sensor for uric acid (UA) and nitric acid (NIT) detection. For monitoring UA and NIT, they used differential-pulse voltammetry and multiple-pulse amperometry. Where this sensor showed LODs of 0.02 and $0.03 \mu \mathrm{mol} \mathrm{L}{ }^{-1}$, a linear range from 0.5 to $250 \mu \mathrm{mol} \mathrm{L}{ }^{-1}$ for both analytes, this is the first study in which 3D-printed sensors and biosensors have been reported for the evaluation of real biological fluids [75]. In addition to the Rafel group approach, the Yang group came up with the idea of a laser-engraved wearable bio-sensor capable of being manufactured on a large scale for the tracking of uric acid (UA) and tyrosine (TYR). This device comprises a laserengraved graphene chemical sensor (LEG-CS) on polyimide for tracking the low level of UA and TYR, a laser-engraved multi-inlet micro-fluidic module for dynamic sweat sampling, and a laser-engraved physical sensor (LEG-PS) for monitoring temperature and breathing. Two laser engraving modes are 


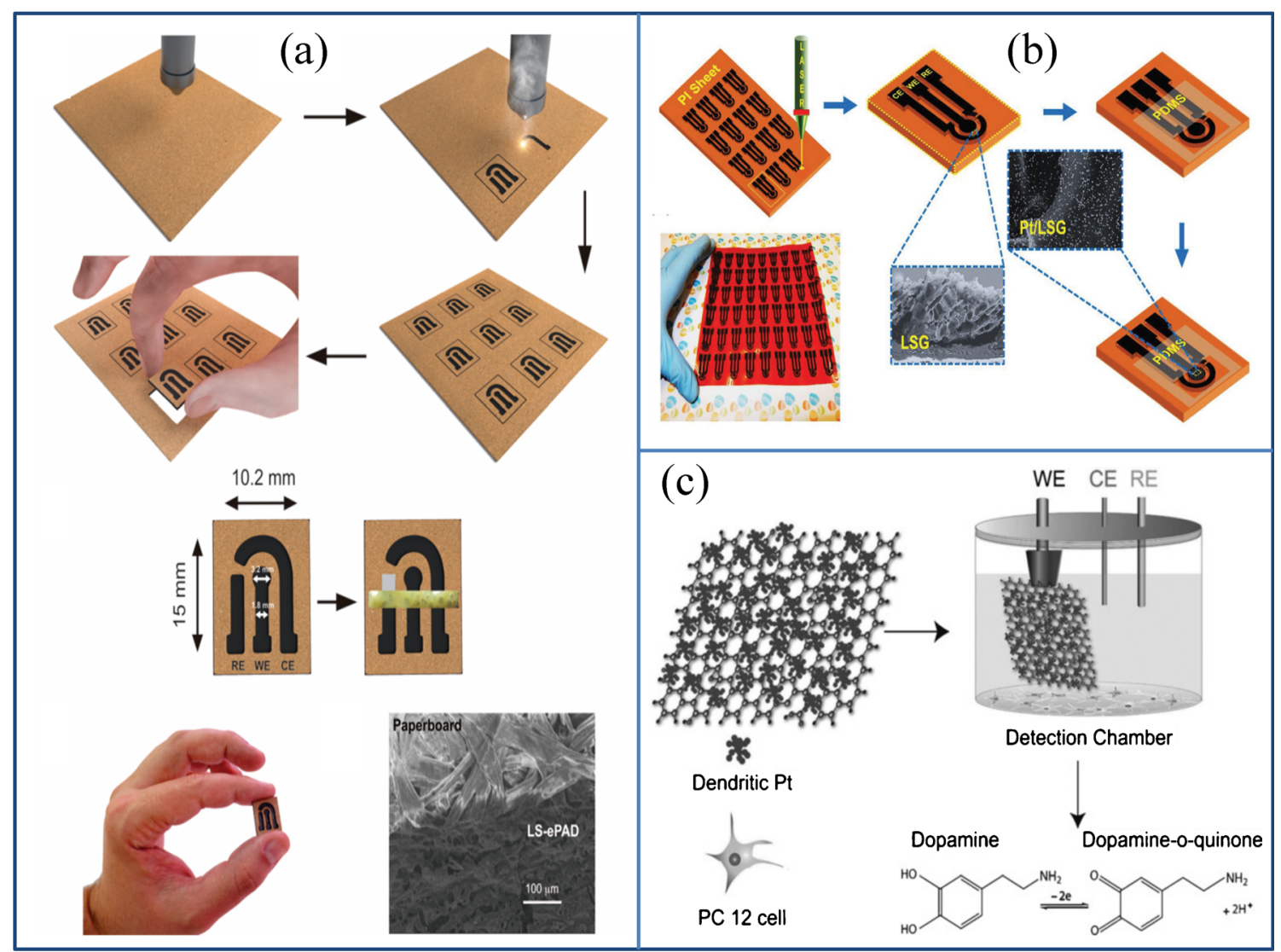

Fig. 6 Flexible as well as wearable sensor for detection of small molecules like ascorbic acid, uric acid, and so on. a Manufacturing of laser scribed paper board (source: reprinted from Thiago R. L. C. Paixão, Lúcio Angnes, José R. Silva, et al, Single-step reagentless laser scribing fabrication of electrochemical paper-based analytical devices, Angewandte Chemie, 129 (2017) 5, with permission from Elsevier). b LSG and Pt/LSG electrode patterns on PI sheet (Source: Reprinted from Pranati Nayak, Narendra Kurra, Chuan Xia, and Husam N. Alshareef.Highly efficient laser scribed graphene electrodes for on-chip electrochemical sensing application, Advanced Electronic Materials, 2 (2016)

in place: raster mode for electrochemical sensors and vector mode for physical and micro-fluidic sensors. The sensitivity of this sensor for UA and Tyr was 3.50 and

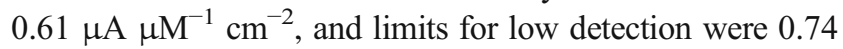
and 3.6 $\mu \mathrm{M}$, respectively. This sensor based on LEG showed excellent selectivity compared to other sweat analyses at physiologically appropriate levels. Additional studies may very well assess its performance in the monitoring of gout, cardiovascular disease, type 2, and kidney disease [63]. Zan et al. demonstrated Fig. $6 \mathrm{c}$ the development of a unique nanohybrid electrode by cooperating two-dimensional dendritic Pt nanoparticles (PtNPs) on free-standing and versatile GPs via a simple dip-coating approach, demonstrating high performance in DA detection produced from living cells (LOD, $5 \mathrm{nM})$ [76].

A flexible rGO-based microelectrode array (MEA) was proposed by Nag et al. using the modified nanoimprint lithography (NIL) technique to track DA. GO was initially prepared
1-11, with permission from Elsevier). c Diagrammatic representation of $\mathrm{Pt} / \mathrm{rGO}$ sensor fabricated by Zan et al. for detection of DA secreted by live PC 12 cells (source: reprinted from Xiaoli Zan Hongwei Bai, Chenxu Wang, Faqiong Zhao, and Hongwei Dua - Graphene paper decorated with a $2 D$ array of dendritic platinum nanoparticles for ultrasensitive electrochemical detection of dopamine secreted by live cells-, Chemsitry, 22 (2016) 5240-5210,https://creativecommons.org/licenses/ by-nc-nd/4.0/, https://chemistryeurope.onlinelibrary.wiley.com/doi/full/ 10.1002/chem.201504454

by the modified hummer's process and spin-coated on a conductive indium tin oxide/polyethylene terephthalate (ITO PET) layer. This NIL process is accompanied by an electrochemical reduction to acquire the final MEA based r-GO sensor. In DA sensing, as well as in tyrosine and $\mathrm{H}_{2} \mathrm{O}_{2}$, the asprepared MEA showed a high signal-to-noise ratio (SNR). When the MEA-based rGO was integrated into a microfluidic system, the sensitivity was virtually unchanged in a continuous-flow environment [77]. This sensor also exhibits good wearability due to its excellent mechanical stability.

There are numerous reports, particularly in twodimensional materials like graphene, on the paper-based flexible substratum $[40,78,79]$. Usually, the combination of graphene with other inorganic nanomaterials like Pt helps to enhance signals from the electrochemical sensor. Here, Duan's group decorated $\mathrm{rGO}$ with $\mathrm{Pt}$ to facilitate the electron transmission reactions of $\mathrm{H}_{2} \mathrm{O}_{2}$. Sheets of metal-metal oxide nanostructures were incorporated into the graphene structures 
to improve the sensitivity of this sensor. The sensitivity was substantially increased to $129.5 \mu \mathrm{A} \mathrm{cm}^{-2} \mathrm{mM}^{-1}$ depending on the metal-metal oxide architecture combined with the mechanical and electrical characteristics of the rGO system and the large surface area of the $\mathrm{MnO}_{2}$ networks as well as the well-distributed catalytic activity of the Pt nanoparticles. This sensor exhibited a linear dynamic range of $2.0 \mu \mathrm{M}$ to $13.33 \mathrm{mM}$ and detection limit down to $1.0 \mu \mathrm{M}$. Figure 7 shows the fabrication procedure involved in the fabrication of this sensor with cyclic voltammetry (CV) curve and amperometric responses, where the CV curve shows a well-described oxidation/reduction peak associated with the $\mathrm{H}_{2} \mathrm{O}_{2}$ oxidation and reduction. The sensitivity of this $\mathrm{H}_{2} \mathrm{O}_{2}$ sensor remained the same, even after $180^{\circ}$ bending. They used the sensor to detect $\mathrm{H}_{2} \mathrm{O}_{2}$ secreted by live cells in real-time studies [80]. Similar to this research, a nonenzymatic bio-sensor based on free-standing rGO paper altered with $\mathrm{Pt}$ nanoparticles (PtNPs) is reported by Song et al. The sensor showed a linear range of 0.2 to $8.5 \mathrm{mmol} / \mathrm{L}$ with a detection limit of around $100 \mathrm{n} / \mathrm{mol}$ [81]. Another versatile paperbased sensor was developed by the Duans group based on a simple and cost-effective dip-coating method shown to detect $\mathrm{H}_{2} \mathrm{O}_{2}$ secreted by live cells. This versatile sensor consists of a two-dimensional array of gold nanoparticles transferred to a freestanding graphene oxide paper at oil-water interfaces, resulting in a single layer of thick, uniformly packed gold nanoparticles mounted on graphene oxide. This sensor also proved to be an effective glucose sensor that shows a linear range of $0.01-46 \mathrm{mM}$ in glucose concentration with a sensitivity of $52.36 \mu \mathrm{A} \mathrm{cm} \mathrm{cm}^{-1}$ and a detection limit of $5 \mu \mathrm{M}$. Such a sensor was also used for real-time $\mathrm{H}_{2} \mathrm{O}_{2}$ monitoring of live cells in a linear range of $0.005-8.6 \mathrm{mM}$, with a sensitivity of $236.8 \mu \mathrm{A} \mathrm{cm} \mathrm{mM}^{-1}$ and a detection limit up to $2 \mu \mathrm{M}$. This sensor's amperometric response, with the subsequent addition of $\mathrm{H}_{2} \mathrm{O}_{2}$, is given in Fig. 8. This simple strategy may help to develop flexible bionano-electronics and energy storage systems for the next generation [82]. Liu et al. reported a flexible non-enzymatic graphene electrode-based sensor for monitoring $\mathrm{H}_{2} \mathrm{O}_{2}$ secretion of live cells. They reported a simple green approach to produce a new form of $\mathrm{MnO}_{2}$ nanowire-graphene hybrid paper using an electrochemical method. This flexible electrode demonstrated strong electrocatalytic behavior towards the redox response of $\mathrm{H}_{2} \mathrm{O}_{2}$. This fabricated sensor showed a linear response to $\mathrm{H}_{2} \mathrm{O}_{2}$ concentration from 0.1 to $45.4 \mathrm{mM}$, with a LOD of $10 \mu \mathrm{M}$ and a sensitivity of $59.0 \mu \mathrm{A} \mathrm{cm}^{-2} \mathrm{mM}^{-1}$. The efficiency of this sensor can be credited to the excellent interaction and excellent interfacial contact between the graphene and the $\mathrm{MnO}_{2}$ nanowires, thus providing a more active center for its electrochemical reactions as well as for the rapid transport of electrons. Thanks to its inherent flexibility, customizable shapes, and versatile properties, this graphenebased nanohybrid paper electrode can be used to produce a high-performance flexible electrode for clinical studies and environmental monitoring purposes [83]. Similar to those observations made by Liu et al., another group reported a non-enzymatic $\mathrm{MnO}_{2}$ /graphene microelectrode sensor for tracking the concentration of $\mathrm{H}_{2} \mathrm{O}_{2}$. They fabricated an $\mathrm{Au} / \mathrm{MnO}_{2} /$ graphene-coated carbon fiber (CF) $\left(\mathrm{Au} / \mathrm{MnO}_{2} / \mathrm{ERGO} / \mathrm{CF}\right)$ nanostructure by in situ electrochemical synthesis, which provided a high level of catalytic efficiency and sustainability, making it useful to detect biologically essential molecules effectively and ultra-sensitively [66]. Ionic liquid molecules can interact with nanosheets of graphene by bonding covalently and non-covalently; the resulting IL-modified nanohybrid materials can effectively enhance electrochemical and hydrophilic properties and make them appropriate electrical materials for light weight and versatile supercapacitors [84]. Based on these concepts, Sun et al. manufactured a flexible nanohybrid electrode paper with the help of an ultrasonic electrodeposition where PtPd alloy nanoparticles are deposited on a freestanding imidazolium-based IL-functional graphene paper for the detection of $\mathrm{H}_{2} \mathrm{O}_{2}$. This manufactured sensor possesses a large electrochemical active surface area (ECSA) with an excellent electrocatalytic performance. This sensor showed a linear range from 0.1 to $37.6 \mu \mathrm{M}$ and a detection limit of about $0.01 \mu \mathrm{M}$ [85]. Compared to research carried out by Cai et al., Peng and the group reported another fiber-based graphene/gold nanosheet composite (GF/AuNS) microelectrode (Fig. 9a) for non-enzymatic glucose and $\mathrm{H}_{2} \mathrm{O}_{2}$ detection. A high $\mathrm{H}_{2} \mathrm{O}_{2}$ and glucose sensing with a low detection limit of 1.62 and $1.15 \mu \mathrm{M}$, and a high sensitivity of 378.1 and $1045.9 \mu \mathrm{A} \mathrm{mM}^{-1} \mathrm{~cm}^{-2}$, respectively, were observed with this sensor. This sensor also exhibited strong biocompatibility that is desirable for several practical applications, including wearable and implantable sensing [86]. Kiransan et al. reported another flexible and selfcontained $\mathrm{Cat}-\mathrm{Fe}_{3} \mathrm{O}_{4} /$ reduced graphene oxide (rGO) hybrid paper as an amperometric $\mathrm{H}_{2} \mathrm{O}_{2}$ sensor and its fabrication procedure as well as $\mathrm{H}_{2} \mathrm{O}_{2}$ electrocatalytic reduction given in Fig. 9b. This electrode showed longterm stability, quick response time, physical deformation tolerance, flexibility, and reproducibility [87]. In addition to these commonly used two-dimensional materials, Ling et al. came up with a flexible biosensor based on a metal-organic framework (MOF) for the detection of small molecules like L-histidine and ascorbic acid (AA), as well as $\mathrm{H}_{2} \mathrm{O}_{2}$. This novel $\mathrm{Cu}$-based MOF is formulated through a coordination bond between two adjacent cupric ions $\left(\mathrm{Cu}^{2+}\right)$ and carboxylic oxygen groups, which leads to a layered structure that is 
Table 1 Performance comparison of enzymatic and non-enzymatic biosensors based on two-dimensional materials

\begin{tabular}{|c|c|c|c|c|c|c|c|}
\hline Electrode & \multicolumn{2}{|l|}{ Linear range } & \multicolumn{2}{|l|}{ Sensitivity } & \multicolumn{2}{|c|}{ Limit of detection } & Ref \\
\hline \multicolumn{8}{|l|}{ Glucose sensors } \\
\hline $\mathrm{Pt} / \mathrm{Co} / \mathrm{NPG} / \mathrm{Go}$ & \multicolumn{2}{|l|}{$35 \mu \mathrm{M}-30 \mathrm{mM}$} & \multicolumn{2}{|l|}{$7.84 \mu \mathrm{A} \mathrm{cm} \mathrm{cm}^{-1}$} & \multicolumn{2}{|l|}{$5 \mu \mathrm{M}$} & [39] \\
\hline PtAu/graphene-CNT-IL/GP & \multicolumn{2}{|l|}{$0.1-11.6 \mathrm{mM}$} & \multicolumn{2}{|l|}{$0.19 \mathrm{~mA} \mathrm{~cm}{ }^{-2} \mathrm{Mm}^{-1}$} & \multicolumn{2}{|l|}{$80 \mu \mathrm{M}$} & [40] \\
\hline $\mathrm{GO}_{x} / \mathrm{PB} /$ graphene hybrid & \multicolumn{2}{|l|}{$0.01-0.7 \mathrm{mM}$} & \multicolumn{2}{|l|}{$1 \mu \mathrm{A} \mathrm{Mm}{ }^{-1}$} & \multicolumn{2}{|l|}{$10 \mu \mathrm{M}$} & [41] \\
\hline Pt-decorated graphite & \multicolumn{2}{|l|}{$0-9 \mathrm{mM}$} & \multicolumn{2}{|l|}{$0.69 \pm 0.06 \mu \mathrm{A} \mathrm{cm}{ }^{-2} \mathrm{Mm}^{-1}$} & \multicolumn{2}{|l|}{$6.6 \mathrm{mM}$} & [42] \\
\hline $\mathrm{Au} /$ graphene/AuNPs/GOD & \multicolumn{2}{|l|}{$0-40 \mathrm{mg} / \mathrm{dL}$} & \multicolumn{2}{|c|}{$667 \mathrm{nA} / \mathrm{mM}$} & \multicolumn{2}{|l|}{$0.3 \mathrm{mg} / \mathrm{dL}$} & [43] \\
\hline LIG electrode & \multicolumn{2}{|l|}{$2.1 \mathrm{mM}$} & \multicolumn{2}{|l|}{$4.622 \mu \mathrm{A} \mathrm{Mm}^{-1}$} & \multicolumn{2}{|l|}{$300 \mathrm{~nm}$} & [46] \\
\hline $\mathrm{rGO} / \mathrm{AuPtNP}$ & \multicolumn{2}{|l|}{$0-2.4 \mathrm{mM}$} & \multicolumn{2}{|l|}{$48 \mu \mathrm{A} \mathrm{cm}{ }^{-2} \mathrm{Mm}^{-1}$} & $5 \mu \mathrm{M}$ & & [48] \\
\hline $\mathrm{MoS}_{2} / \mathrm{AuNPs} /$ glucose oxidase (GOx) & $500-100 \mathrm{nM}$ & & & & $10 \mathrm{nM}$ & & {$[50]$} \\
\hline $\mathrm{NiSe}_{2}$-based glucose sensors & $0.1-1 \mathrm{mM}$ & & $0.25 \mathrm{AM}^{-1} \mathrm{~cm}^{-2}$ & & $24.8 \mu \mathrm{M}$ & & [51] \\
\hline $\mathrm{CuCo}_{2} \mathrm{O}_{4} /$ graphite & $0-320 \mu \mathrm{M}$ & & $3.625 \mu \mathrm{A} \mu \mathrm{M}^{-1} \mathrm{~cm}^{-2}$ & & $5 \mu \mathrm{M}$ & & [52] \\
\hline Lactate sensors & & & & & & & \\
\hline $\mathrm{GP}-\mathrm{MoS}_{2}-\mathrm{Cu}-\mathrm{LOD}$ & $5-1775 \mu \mathrm{M}$ & & $3.38 \mathrm{~mA} \mathrm{~cm}{ }^{-2} \mathrm{Mm}^{-1}$ & & $500 \mathrm{nM}$ & & [49] \\
\hline $\mathrm{CNTs} / \mathrm{Ti}_{3} \mathrm{C}_{2} \mathrm{~T}_{x} / \mathrm{PB} / \mathrm{CFM}$ & $10 \times 10^{-6}-22 \times 10^{-3} \mathrm{M}$ & & $11.4 \mu \mathrm{A} \mathrm{mM}^{-1}$ & & $0.67 \times 10^{-6} \mathrm{M}$ & & [59] \\
\hline $\mathrm{Cu}$ NPs-LIG & $1 \mu \mathrm{M}-6 \mathrm{mM}$ & & $495 \mu \mathrm{A} \mathrm{Mm}{ }^{-1} \mathrm{~cm}^{-2}$ & & $0.39 \mu \mathrm{M}$ & & [63] \\
\hline Biologically relevant molecules & & & & & & & \\
\hline $\mathrm{GF} / \mathrm{NiCo}_{2} \mathrm{O}_{4}$ & $200-750 \mu \mathrm{M}$ & AA & & & $50 \mu \mathrm{M}$ & AA & [70] \\
\hline & $1-13 \mu \mathrm{M}$ & DA & & & $0.1 \mu \mathrm{M}$ & DA & \\
\hline & $10-26 \mu \mathrm{M}$ & UA & & & $0.2 \mu \mathrm{M}$ & UA & \\
\hline $\mathrm{Pt} / \mathrm{LSG}$ & $10-890 \times 10^{-6} \mathrm{M}$ & AA & $250.69 \mu \mathrm{AmM}^{-1} \mathrm{~cm}^{-2}$ & AA & $6.1 \times 10^{-6} \mathrm{M}$ & $\mathrm{AA}$ & [74] \\
\hline & $0.5-56 \times 10^{-6} \mathrm{M}$ & DA & $6995.6 \mu \mathrm{A} \mathrm{mM}{ }^{-1} \mathrm{~cm}^{-2}$ & DA & $0.07 \times 10^{-6} \mathrm{M}$ & DA & \\
\hline & $1-63 \times 10^{-6} \mathrm{M}$ & UA & $8289 \mu \mathrm{AmM}^{-1} \mathrm{~cm}^{-2}$ & UA & $0.22 \times 10^{-6} \mathrm{M}$ & UA & \\
\hline G-PLA & $0.5-250 \mu \mathrm{A} \mathrm{L} \mu \mathrm{mol}^{-1}$ & UA & $0.1332 \mu \mathrm{A} \mathrm{L} \mu \mathrm{mol}^{-1}$ & UA & $0.02{\mu \mathrm{mol}^{-1}}^{-1}$ & UA & [75] \\
\hline & $0.5-250 \mu \mathrm{A} \mathrm{L} \mu \mathrm{mol}^{-1}$ & NIT & $0.0922 \mu \mathrm{A} \mathrm{L} \mu \mathrm{mol}^{-1}$ & NIT & $0.03{\mu \mathrm{mol}^{-1}}^{-1}$ & NIT & \\
\hline LEG-CS & & & $3.50 \mu \mathrm{A} \mu \mathrm{M}^{-} \mathrm{cm}^{-2}$ & UA & $0.74 \mu \mathrm{M}$ & UA & [63] \\
\hline & & & $0.61 \mu \mathrm{A} \mu \mathrm{M}^{-} \mathrm{cm}^{-2}$ & Tyr & $3.6 \mu \mathrm{M}$ & Tyr & \\
\hline $\mathrm{Pt} / \mathrm{rGO}$ paper & $87 \mathrm{~nm}-100 \mu \mathrm{M}$ & DA & $2 \mu \mathrm{A} \mathrm{cm} \mathrm{mm}^{-1}$ & DA & $5 \mathrm{~nm}$ & DA & [76] \\
\hline rGO MEA device & & DA & $12.7 \mathrm{nA} \mu \mathrm{M}^{-1}$ & DA & $0.1 \mu \mathrm{M}$ & DA & [77] \\
\hline & & Tyro & $0.34 \mathrm{nA} \mu \mathrm{M}^{-1}$ & Tyro & $3.7 \mu \mathrm{M}$ & Tyro & \\
\hline $\mathrm{MoS}_{2} / \mathrm{rGO}$ & $0.1-240 \mu \mathrm{M}$ & FA & $230 \mu \mathrm{A} \mathrm{mM}^{-1}$ & FA & $3.68310^{-8} \mathrm{M}$ & FA & [78] \\
\hline Co-MOF & $10-2400 \mu \mathrm{M}$ & AA & & & $2.94 \mu \mathrm{M}$ & AA & [88] \\
\hline $\mathrm{H}_{2} \mathrm{O}_{2}$ sensing & & & & & & & \\
\hline $\mathrm{Au} / \mathrm{MnO}_{2} /$ graphene-coated $\mathrm{CF}$ & $0.05-14.2 \mathrm{mM}$ & & $0.167 \mathrm{~mA} \mathrm{~cm}^{-2} \mathrm{mM}^{-1}$ & & $2 \mu \mathrm{M}$ & & [66] \\
\hline $\mathrm{Pt} / \mathrm{MnO}_{2} / \mathrm{rGOP}$ & $2.0 \mu \mathrm{M}-13.33 \mathrm{mM}$ & & $129.5 \mu \mathrm{A} \mathrm{cm}^{-2} \mathrm{mM}^{-1}$ & & $1 \mu \mathrm{M}$ & & [80] \\
\hline $\mathrm{MnO}_{2} / \mathrm{ERGO}$ & $0.1-45.4 \mathrm{mM}$ & & $59 \mu \mathrm{A} \mathrm{cm}^{-2} \mathrm{mM}^{-1}$ & & $10 \mu \mathrm{M}$ & & [83] \\
\hline Au-Rgo paper & $0.05-8.6 \mathrm{mM}$ & & $236.8 \mu \mathrm{A} \mathrm{cm}^{-2} \mathrm{mM}^{-1}$ & & $2 \mu \mathrm{M}$ & & [82] \\
\hline Cat- $\mathrm{Fe}_{3} \mathrm{O}_{4} / \mathrm{rGO}$ & $3.30 \mu \mathrm{M}-5.56 \mathrm{mM}$ & & $1.84 \mathrm{~mA} \mathrm{~cm}{ }^{-2} \mathrm{mM}^{-1}$ & & $110 \mathrm{nM}$ & & [87] \\
\hline IL-rGO & $0.1-37.6 \mu \mathrm{M}$ & & & & $0.01 \mu \mathrm{M}$ & & [85] \\
\hline GF/AuNS & $9.4 \mu \mathrm{M}-13 \mathrm{mM}$ & & $1045.9 \mu \mathrm{A} \mathrm{cm}^{-2} \mathrm{mM}^{-1}$ & & $1.62 \mu \mathrm{M}$ & & [86] \\
\hline rGO MEA device & & & $2.3 \mathrm{nA} \mu \mathrm{M}^{-1}$ & & $0.35 \mu \mathrm{M}$ & & [77] \\
\hline $\mathrm{Cu}-\mathrm{MOF}$ & $10-1000,1400-6800 \mu$ & & & & $4.1 \mu \mathrm{M}$ & & [88] \\
\hline
\end{tabular}

interlinked with $\mathrm{H}_{2}$ bonding. The MOF sensing mechanism is based on the $\mathrm{Cu}^{2+} / \mathrm{Cu}^{+}$redox capability. The electrode surface of this MOF sensor absorbs the analytes and then oxidizes by active metal $\mathrm{Cu}^{2+}$ sites which resulted in a visible oxidation current that is a measure of the analyte concentration. This sensor also showed excellent bending distortion, therefore compliant with the complex human body surroundings [88]. Table 1 shows the performance comparison of enzymatic/ non-enzymatic flexible and wearable biosensors based on two-dimensional materials so far reported. 


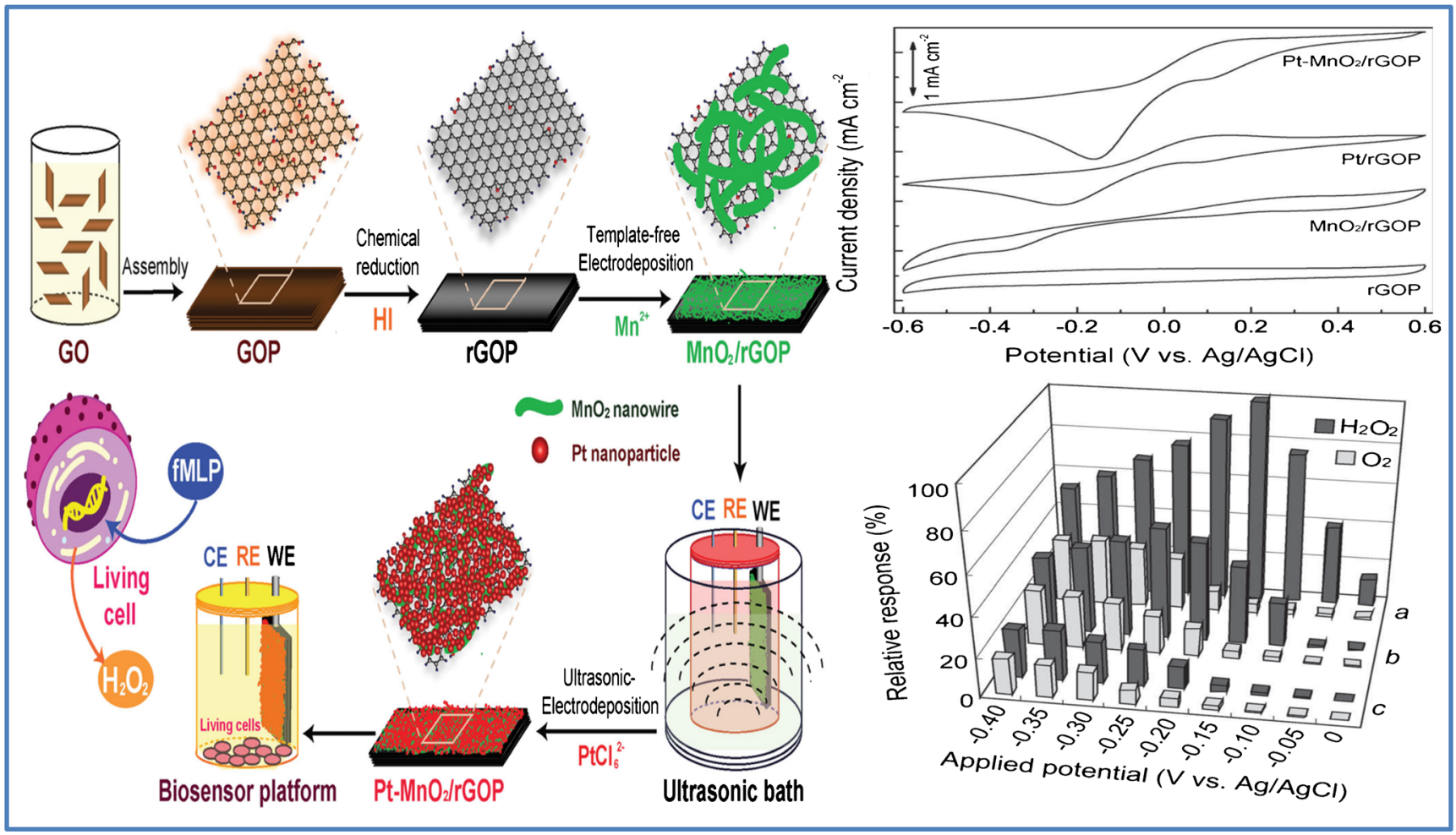

Fig. 7 Two-dimensional material-based flexible/wearable sensor for $\mathrm{H}_{2} \mathrm{O}_{2}$ detection. Diagrammatic representation of metal-metal oxide nanostructures developed on a freestanding graphene paper developed by Duan et al. and the corresponding amperometric response of the sensor (source: reprinted from Fei Xiao, Yuanqing Li, Xiaoli Zan, Kin Liao,

\section{pH sensors}

The $\mathrm{pH}$ values represent the behavior of a variety of physiological, biological, and medical processes, such as enzymes responses, tumor metastases, wound healing, cell growth, etc. The $\mathrm{pH}$ levels of bio-fluids such as urine, tears, sweat, and saliva could, therefore, be assessed in situ to detect certain diseases in advance [89].

\section{Two-dimensional material based wearable/flexible sensor for} the measurement of $\mathrm{pH}$ Various materials, like carbohydrate materials, metals or their oxides, biological enzymes, etc., can produce a chemical reaction by hydrogen ions and there is a greater abundance of sensing electrode materials in $\mathrm{pH}$ sensors that make $\mathrm{pH}$ sensors more focused [90]. Meali et al. developed a GO-based $\mathrm{pH}$ sensor to monitor wound healing. This sensor's sensitivity was found to be around $31.8 \mathrm{mV} / \mathrm{pH}$, with a precision of $0.3 \mathrm{pH}$. Yet this sensor must be further adjusted to achieve the goals of miniaturization, reliability, sensitivity, and reversibility for use in human body treatment [91]. Daniel et al. reported a low-cost potential sensor array made of polymethylmethacrylate (PMMA) polymer, graphene plates, and ruthenium oxide $\left(\mathrm{RuO}_{2}\right)$ sub-microns. This sensor consists of three layers, an ultraviolet (UV)-curable dielectric paste, a low-temperature-curable conductive
Rong Xu, and Hongwei Duan- Growth of metal-metal oxide nanostructures on freestanding graphene paper for flexible biosensor, Advanced Functional Materials 22, (2012)2487-2494, with permission from Elsevier)

silver paste, and a pH-sensitive PMMA/graphene/ruthenium oxide paste produced above flexible polyester shown in Fig. 10a with its calibration curve. After 10,000 cycles of perpendicular bending, the sensor's flexibility and fatigue test show a significant decrease in performance, indicating good durability of the sensor. The sensing mechanism of graphene is given by Lei et al. The equation shown below gives the catalytic reaction of the graphene-based $\mathrm{pH}$ sensor. The sensing mechanism is explained by these adsorbed ions, i.e., $\mathrm{H}_{3} \mathrm{O}^{+}$ and $\mathrm{OH}^{-}$[92].

$\mathrm{H}_{2}+\mathrm{H}_{2} \mathrm{O} \rightarrow \mathrm{H}_{3} \mathrm{O}^{+}+\mathrm{e}^{-}$
$\mathrm{O}_{2}+2 \mathrm{H}_{2} \mathrm{O}+4 \mathrm{e}^{-} \rightarrow 4 \mathrm{OH}^{-}$

The adsorption of hydroxonium $\left(\mathrm{H}_{3} \mathrm{O}^{+}\right)$and hydroxyl ions $\left(\mathrm{OH}^{-}\right)$is non-faradic (capacitive) to prevent charge transfer through the graphene/solution interface. Consequently, the hydroxonium $\left(\mathrm{H}_{3} \mathrm{O}^{+}\right)$makes graphene $\mathrm{N}$-doped, and hydroxyl ions $\left(\mathrm{OH}^{-}\right)$make graphene $\mathrm{P}$-doped depending on the $\mathrm{pH}$ of the solution according to the double-layer configuration of the interface graphene/solution [93]. Here, the performance of the $\mathrm{pH}$ sensing array is affected by additional ions present in sweat, such as $\mathrm{Ca}^{2+}, \mathrm{Na}^{+}, \mathrm{K}^{+}$, etc. [94]. The sensor developed by Lei et al. was briefly given in "Invasive," which can 


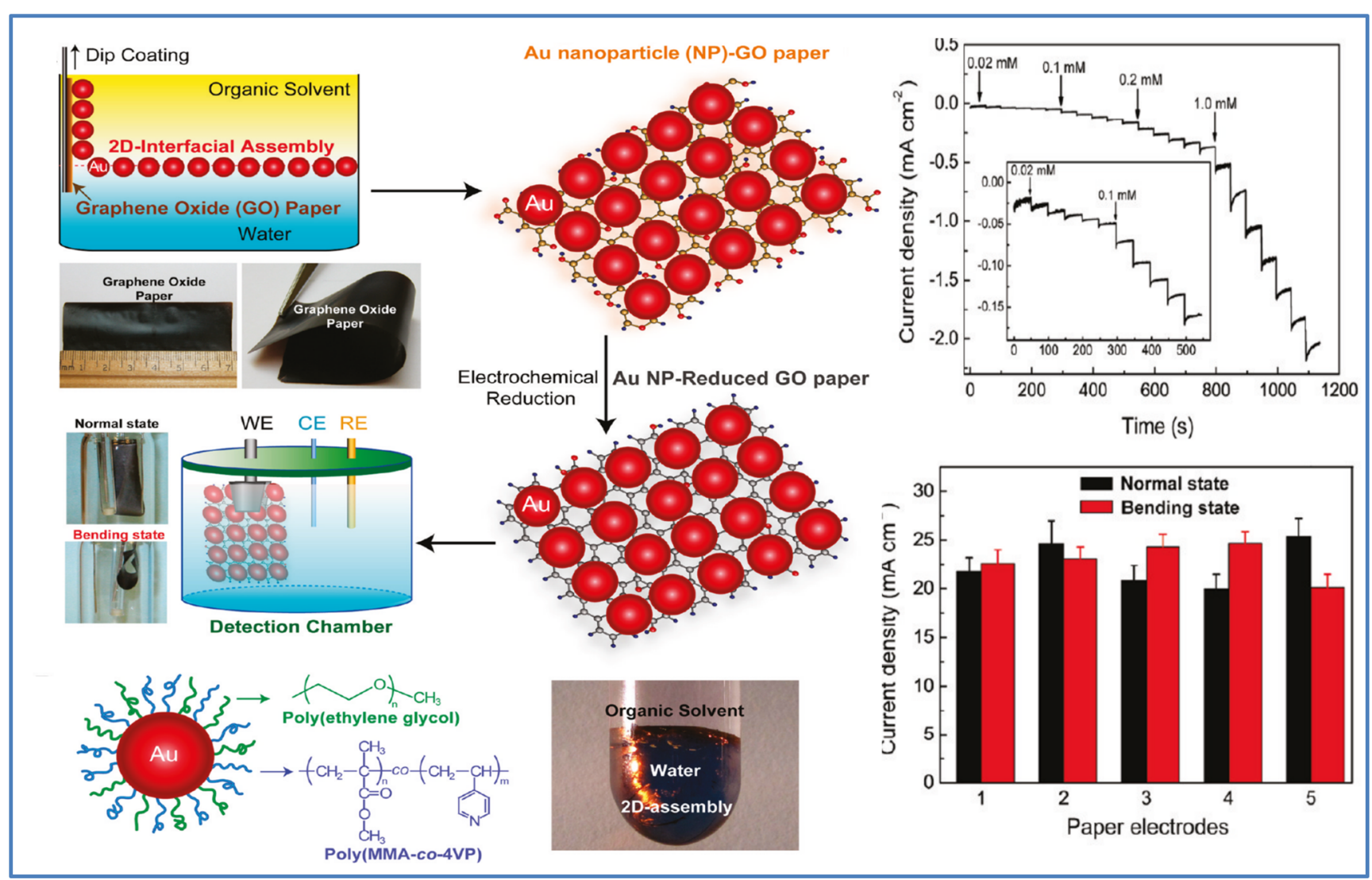

Fig. 8 Two-dimensional material-based flexible/wearable sensor for $\mathrm{H}_{2} \mathrm{O}_{2}$ detection. Diagrammatic representation of fabrication $\mathrm{H}_{2} \mathrm{O}_{2}$ sensor based on the hybrid electrode of $2 \mathrm{D}$ Au particles on graphene paper and corresponding amperometric response of the fabricated sensor (source: reprinted from Fei Xiao, Jibin Song, Hongcai Gao, Xiaoli Zan, Rong Xu, and Hongwei Duan- Coating graphene paper with 2D-assembly of electrocatalytic nanoparticles: a modular approach towards highperformance flexible electrode, ACS Nano, 6 (2012) 100-110, Copyright (2020) American Chemical Society) simultaneously detect $\mathrm{pH}$, glucose, and lactate; here, the $\mathrm{pH}$ sensing part is made up of polyaniline. The sensitivity of this $\mathrm{pH}$ sensor is around $-70 \mathrm{mV} \mathrm{pH}^{-1}$ with good linearity. But the main drawback of this sensor as a $\mathrm{pH}$ sensor is that the $\mathrm{pH}$ range was fairly small because of its enzymatic activity [59]. Tehrani et al. recently manufactured a novel electrochemical $\mathrm{pH}$ sensor based on pigment melanin functionalized with dimethyl sulfoxide (DMSO) pigment melanin (PGM) and graphene developed using a screen printing technique given in Fig. 10b. Here, the binary compound used, i.e., melanin, is a useful electrochemical sensing compound with a recorded sensitivity of approximately $-50 \mathrm{mV} / \mathrm{p}$ and has a feature of $\mathrm{pH}$ [95] that helps to increase the sensitivity of graphene. This sensor showed high sensitivity $(62 \mathrm{mV} / \mathrm{pH} \pm 7)$, with a $\mathrm{pH}$ range of 5 to 8 , which is also very stable and highly efficient compared to several existing equipment [96].

Among the TMDs, nickel diselenide $\left(\mathrm{NiSe}_{2}\right)$ is identified as abundantly present on earth, with most of the $\mathrm{NiSe}_{2}$ reports based on electrochemical applications, and there are rare studies of the use of $\mathrm{NiSe}_{2}$ for electronic sensors. Sushmitha et al. proposed portable wireless, a wearable multi-functional sensor for both physio-chemical stimuli (breath and $\mathrm{pH}$ ) with a single-step growth of nickel diselenide on a paper substrate. This non-invasive saliva $\mathrm{pH}$ sensor of $\mathrm{NiSe}_{2}$ can be used to detect periodontal and gum problems. $\mathrm{NiSe}_{2}$, due to its metallic nature, has free electrons in the conduction band. Since the acidic solutions have more $\mathrm{H}^{+}$ions, it accepts an electron from $\mathrm{NiSe}_{2}$ and the resistance is increased. In comparison, an alkaline solution with $\mathrm{OH}^{-}$ions gives electrons to $\mathrm{NiSe}_{2}$, which enhances the electron free flow and reduces the $\mathrm{pH}$ sensor resistance. The sensor was attached to a microcontroller, and the collected data was transferred to the mobile device via the Bluetooth module. This cheap disposable sensor can easily be used to test the unknown solution's pH (acidic or basic) when an advanced $\mathrm{pH}$ meter is not usable [97]. Similar to the Meali et al. research, several other reports are available on this type of flexible $\mathrm{pH}$ sensor such as amino-functionalized graphene fragments and polyaniline $\left(\mathrm{NH}_{2}-\mathrm{G} / \mathrm{PANI}\right)$ composite film developed by $\mathrm{Su}$ et al. [98], layered $\mathrm{ReS}_{2}$ field-effect transistors (FETs) developed by Liao et al., and so on, but these also need to be further modified to meet miniaturization goals for the practical use of human body diagnosis [99]. 


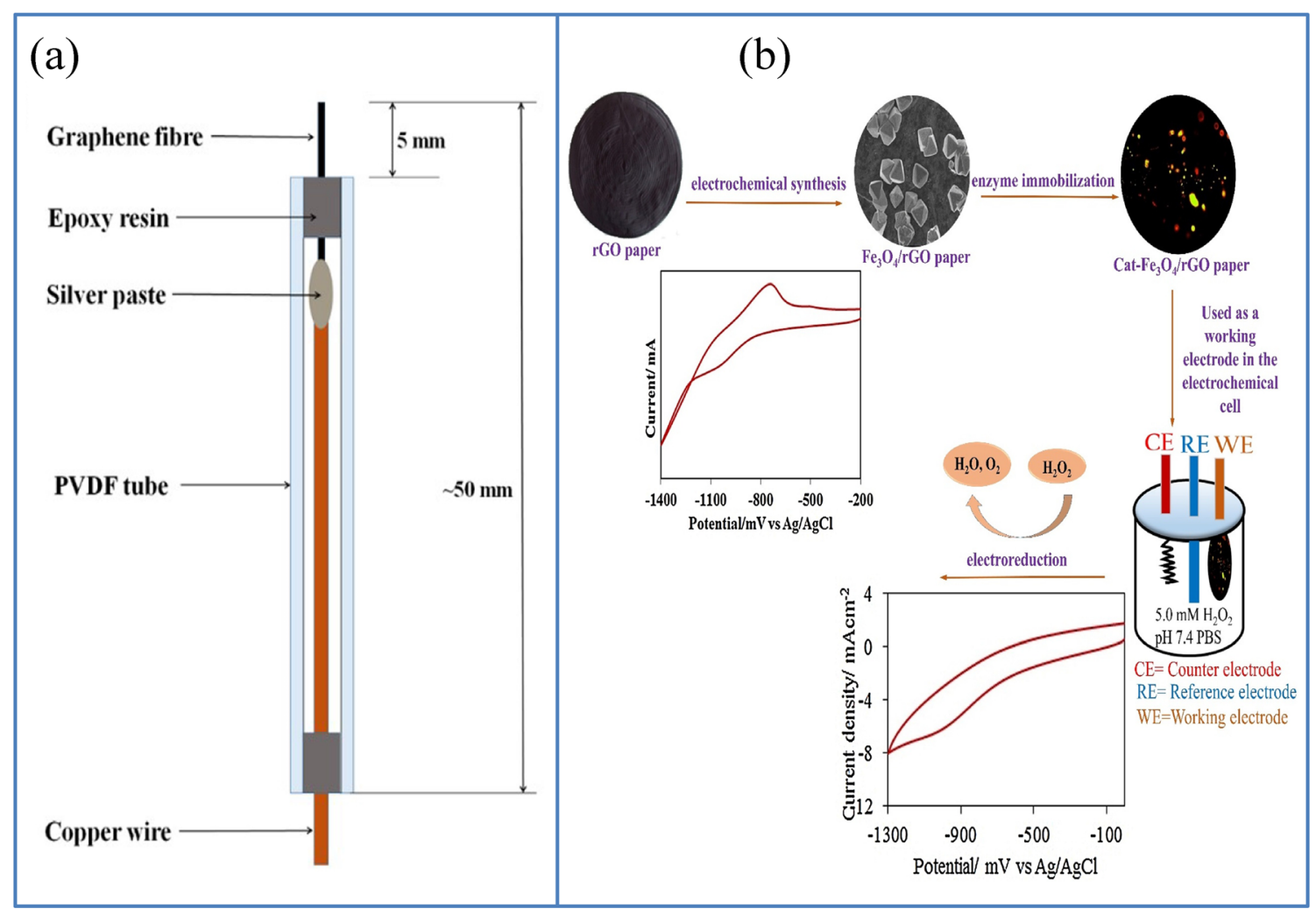

Fig. 9 Two-dimensional material-based flexible/wearable sensor for $\mathrm{H}_{2} \mathrm{O}_{2}$ detection. a Flexible microelectrode based on graphene developed by Peng et al.(Source: reprinted from Peng, Yu Lin, Deqing Justin Gooding, J. Xue, Yuhua Dai, Liming-Flexible fiber-shaped non-enzymatic sensors with a graphene-metal heterostructure based on graphene fibers decorated with gold nanosheets, Carbon (2018) 329-336, with

\section{Affinity biosensors: immunosensors}

Biosensors based on the antigen-antibody interactions on a transducer surface are described as immunosensors. Immunosensors constitute the most important classes of affinity biosensors (devices based on the irreversible binding of receptor molecules to the analyte molecules, detectable physiochemical change is produced) depending on the specific recognition of antigens by antibodies to generate a stable complex, similar to immunoassay [100]. Immunoreactions are characterized by high specificity and selectivity. In immunoreactions, the strong binding forces between the biomolecules allow high sensitivity and specificity. This makes immunosensors a prominent sensing approach in clinical analysis. Most of the immune techniques are characterized by enzyme-linked immunosorbent assay (ELISA), which depends on a solid-state sandwich immunoassay [101].

\section{Principles of immunosensors}

Antibodies are also known as immunoglobulins (Ig), and they constitute a large group of glycoproteins that can specifically recognize the antigens. They comprise single or multiple permission from Elsevier). b Diagrammatic representation of the manufacturing of paper-based Cat- $\mathrm{Fe}_{3} \mathrm{O}_{4} / \mathrm{rGO}$ electrode and $\mathrm{H}_{2} \mathrm{O}_{2}$ electrocatalytic reduction. (Source: reprinted from Kader Dağcl Kıranşan, Mine Aksoy, Ezgi Topçu-Flexible and freestanding catalase$\mathrm{Fe}_{3} \mathrm{O}_{4}$ /reduced graphene oxide paper: enzymatic hydrogen peroxide sensor applications, Materials Research Bulletin, 106(2018) 57-6

copies of characteristic units with a "Y" shape. The structure of Y contains four polypeptide chains in which two are identical light chains and two are identical heavy chains. Each pair of heavy and light chains is connected by single disulfide bonds $[102,103]$. The antibodies are classified into monoclonal (specific to a single epitope) and polyclonal (bind to multiple epitopes and exhibit an immunological response to multiple analytes) $A b$ based on the epitope, which indicates the region of antigen-antibody interaction [104, 105]. The slighter chemical modification in the molecular structure of the antigen can affect the affinity of $A b$. This affinity is used to measure the binding forces in the $A g-A b$ complex. The association or affinity constant can be of $10^{15}$ under normal temperature, $\mathrm{pH}$, and buffer solution [106, 107]. ELISA can be explained as the immobilization of Ag on solid support followed by the transfer of specific enzyme-linked $\mathrm{Ab}$ over the support to connect the $A g$, and at the end, a solution of the enzyme's substrate is added. Commonly, a color change is produced in the reaction, which can be detected optically [108]. Sandwich-type immunosensors contain primary antibodies that are immobilized on the surface of the working electrode, and a sandwiched immunocomplex is produced in between the primary $A b$ (immobilized) and the secondary $A b$ 


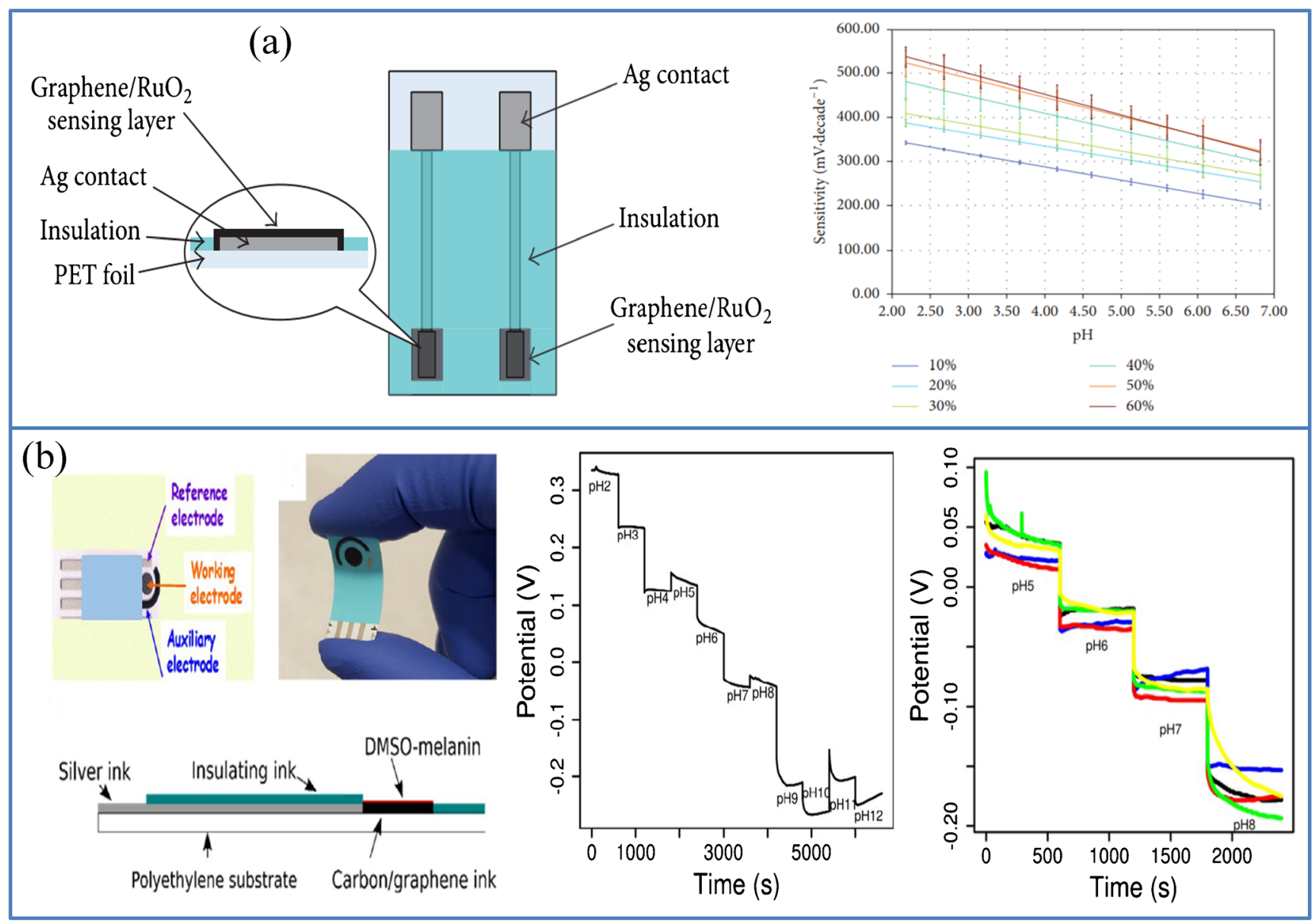

Fig. 10 Flexible as well as wearable $\mathrm{pH}$ sensors. a Schematic diagram of the sensor structure based on graphene $/ \mathrm{RuO}_{2}$ (Daniel Janczak, Andrzej Peplowski, Grzegorz. Wroblewski, Lukasz Gorski, Elzbieta Zwierkowska, and Malgorzata Jakubowska- Investigations of printed flexible pH sensing materials based on graphene platelets and submicron $\mathrm{RuO}_{2}$ powders, Journal of Sensors (2017)1-7, Copyright (C) 2017 Daniel Janczak et al. b Flexible PGM based sensor, its $\mathrm{pH}$ sensing performance and stability (reproduced with permission from Z. Tehrani1, S.P. Whelan1, A B Mostert, J V Paulin, M Ali1, E. Daghigh Ahmadil, C F O Graeff, O J Guy and D T Gethin- Printable and flexible graphene pH sensors utilizing thin film melanin for physiological applications, 2D Mater. In (2020),https://doi.org/10.1088/2053-1583/ab72d5, https:// creativecommons.org/licences/by/3.0 with multiple detections [109]. Sandwich-type immunoassay is a common strategy of immunosensor, and it relies on the separation of unbounded materials without any participation in the binding process. The immunosensor-based detection of analytes is reversible, selective, and continuous due to the higher affinity constants of $A g-A b$ interactions, which is not reversible immediately [110].

In electrochemical transducers (potentiometric, amperometric, and impedimetric), the immunoreactions can produce changes in potential, current, capacitance, or impedance. The electrochemical transducers are characterized by cost-effectiveness, robustness, fast response time, and fewer analyte volumes, and can be widely used as immunosensors for several applications [101]. A variety of analytes from the clinical field can be effectively detected by the coupling between immunological reactions and amperometric electrodes. The potentiometric immunosensors are based on the measurement of change in potential before and after the $\mathrm{Ag}-\mathrm{Ab}$ interaction. The net electric charge polarity of either antibodies or antigen is correlated with the ionic composition of the solution and the isoelectric points of components. The impedimetric immunosensors rely on the impedance changes of the electrode before and after the immunoreactions, which is to correlate the concentration of $A g$ or $A b$ in the analyte sample solution. Currently, the onsite analysis of target molecules can be possible by the exploration of simple, flexible, and miniaturized biosensors based on the sensing platform of electroanalytical techniques [101]. 


\section{Flexible electrochemical immunosensors based on two-dimensional materials for clinical analysis}

\section{Polymer-based immunosensors}

Graphene is an important material for the development of sensor devices owing to high reactivity, biocompatibility, high Young's modulus, high surface area, distinctive optical and electrical properties, and the possibility of surface functionalization with biomolecules [111]. Moreover, it is an excellent material candidate to fabricate cost-effective sensors on flexible substrates. The chemical vapor deposition techniques for the fabrication of traditional graphene devices are too expensive for electrochemical sensor applications; lowcost alternative methods including inkjet and screen printing do not have adequate control over the geometry of the electrode to attain the convenient electrochemical sensing performance. Kshama et al. fabricated interdigitated electrodes (IDEs) of graphene with high resolution of $40 \mu \mathrm{m}$ line width on a flexible polyimide (Kapton) substrate for the sensing of histamine in contaminated food through the aerosol jet printing (AJP) method. This IDE pattern of graphene is converted to histamine sensors through covalent binding of antibodies (monoclonal) to oxygen species produced on the graphene surface via the thermal annealing process of $\mathrm{CO}_{2}$ at $400{ }^{\circ} \mathrm{C}$. The thermal annealing process is explored to functionalize graphene surface using carbonyl and carboxyl groups, to improve the antibody binding on graphene IDEs. The AJP method is a high resolution and high yield direct-write printing method, which do not require any additional fabrication steps to create high resolution features for sensing device without auxiliary patterning (Fig. 11a) [112]. Moreover, the sensing activity was not affected by blocking agents such as protein molecules that are usually seen in food samples (Fig. 11b). The Nyquist plots for the various histamine concentrations in the fish broth sample are shown in Fig. 11c. Histamine present in the tuna fish broth sample was monitored by measuring the variation in charge transfer resistance of the graphene IDE sensor as a function of histamine concentration Fig. 11d. The synthesized electrochemical sensor showed enhanced

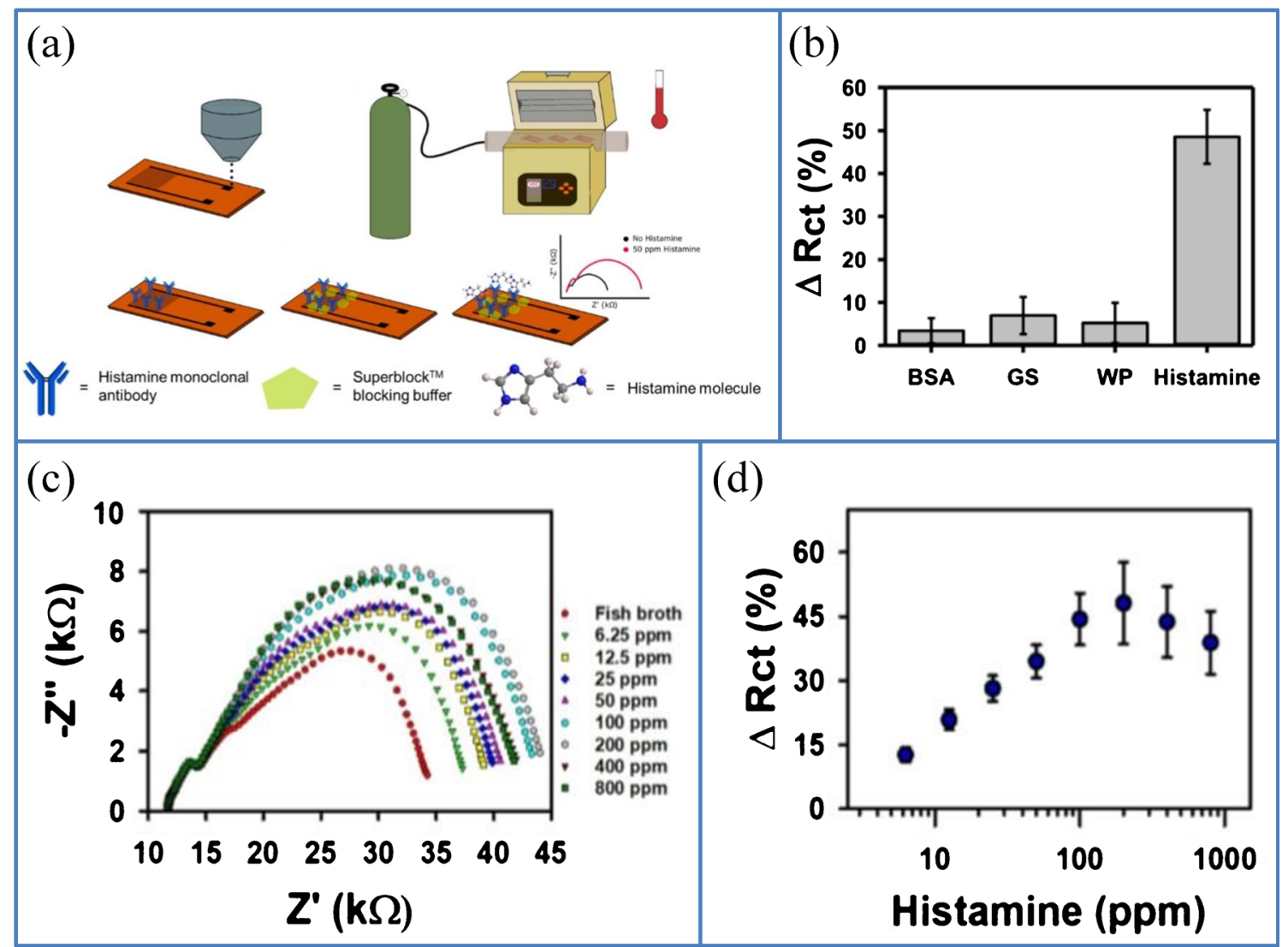

Fig. 11 a Fabrication and functionalization of AJP graphene IDE sensor, which includes aerosol jet printing of graphene in PI substrate, thermal annealing process to generate oxygen-rich species on the graphene IDE sensor, immobilization of desired antibodies on the graphene surface, and covering of unfunctionalized areas of the graphene IDE sensor using a blocking agent to maintain the selectivity. $\mathbf{b}$ Non-specific adsorption test of AJP graphene IDE modified with histamine antibody against other interfering protein molecules usually present in food samples, which can be used as blocking agents that can interfere with the antibody activity. $\mathbf{c}$ Nyquist plots for different concentrations of histamine in fish broth sample and $\mathbf{d}$ calibration plot of change in charge transfer resistance $\mathrm{v} / \mathrm{s}$ histamine concentrations in fish broth sample. (Source: reprinted from Parate K, Pola CC, Rangnekar S V., Mendivelso-Perez DL, Smith EA, Hersam MC. Aerosol-jet-printed graphene electrochemical histamine sensors for food safety monitoring. 2D Mater. 2020(1-13);7) 
sensing performance to histamine in a range of $56.25-1.8 \mu \mathrm{M}$ $(6.25-200 \mathrm{ppm})$ with a low detection limit of $30.7 \mu \mathrm{M}$ $(3.41 \mathrm{ppm})$ and response time of $33 \mathrm{~min}$ in samples of tuna broth without any pre-treatment and pre-labeling. Histamine levels above $50 \mathrm{ppm}$ in a fish sample can cause severe allergic reactions (scromboid food poisoning). The graphene-based histamine sensor (histamine-biogenic amine found in spoiled fish products and seafood allergies) is appropriate for the infield monitoring. Apart from the histamine sensing, the AJP and functionalization can be popularized to various sensing applications like food toxin detection, flexible and wearable health-monitoring devices, environmental toxins, and health care diagnostics. Additionally, this rapid immunosensor can be used for applications including food processing, food transportation, and supermarkets where the regular on-site analysis of food samples is needed to confirm and maintain the food quality. This on-site analysis of food items will avoid the need for laboratory food testing because the lab-testing protocols are needed additional handling steps, which is time consuming and costly, and increases the possibility of food wastage and foodborne diseases [113].

Nowadays, the spoilage of food due to the presence of microorganisms like bacteria and viruses is becoming a global concern all over the world. Most of the microorganisms like bacteria, fungi, viruses, bio-toxins, and other microspecies are unsusceptible to environmental conditions. On the contrary, humans are quite prone to most common infectious diseases caused by foodborne pathogens or microorganisms such as Escherichia coli, Salmonella sp., and Bacillus cereus are still higher in many countries. Another successful flexible and highly sensitive electrochemical immunosensor was fabricated by Liu et al. using conductive graphene film with the freestanding ability for the detection of the rotavirus (Fig. 12a). They fabricated graphene film using the centrifugal evaporation method, and the construction process of immunosensor based on a three-electrode CV system with free-standing graphene film as the working electrode is shown in Fig. 12b.

Moreover, this free-standing film of graphene exhibited a highly dense packing of graphene sheets with a characteristic ripple structure on the surface, and showed fast electron transfer and high conductivity property, making it advantageous to be applied as a working electrode in electrochemical analytical techniques. The graphene film was modified with pyrene derivatives to covalently bind with the rotavirus-specific antibodies, which is immobilized on the electrode surface, as shown in Fig. 12c. The entire sensing process was monitored

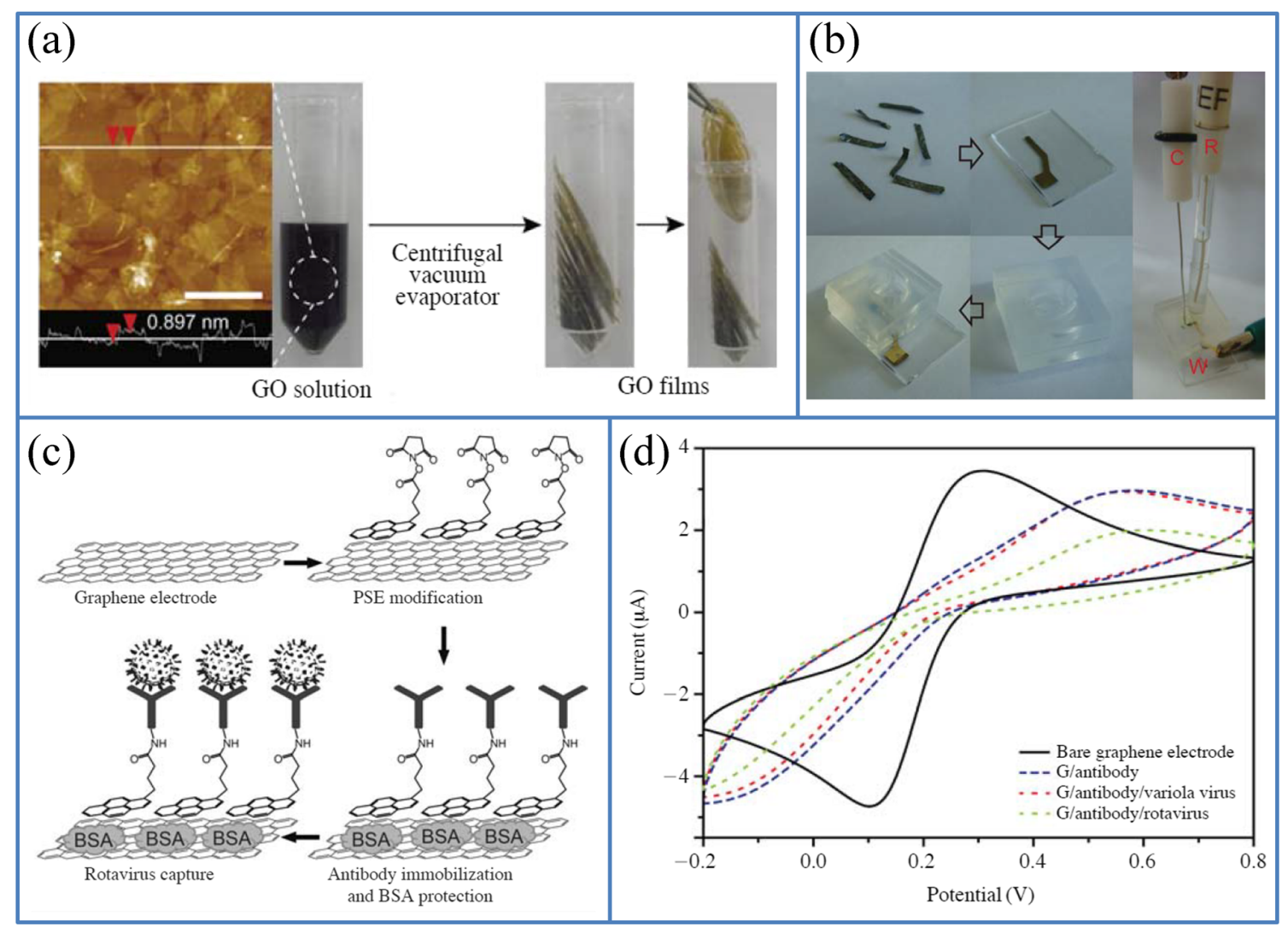

Fig. 12 Photographs of the a fabrication of graphene films using the centrifugal vacuum evaporation and $\mathbf{b}$ fabrication of a three-electrode system of CV with graphene film as a working electrode. c Schematic image of the fabricated immunosensor based on graphene film for the detection of rotavirus. $\mathbf{d ~ C V}$ of the bare graphene electrode, $\mathrm{G} / \mathrm{Ab}, \mathrm{G} / \mathrm{Ab} /$

rotavirus $\left(10^{5} \mathrm{pfu} / \mathrm{mL}\right)$ and $\mathrm{G} / \mathrm{Ab} /$ variola virus used as a negative control. (Source: reprinted from Liu F, Choi K S, Park T J, Lee S Y, Seo T S. Graphene-based electrochemical biosensor for pathogenic virus detection. Biochip J. 2011;5:123-128) 
by cyclic voltammetry, and the rotavirus is adsorbed on the graphene film via antigen-antibody interaction. The fabricated electrochemical immunosensor showed a high sensitivity of $30.7 \%$ to rotavirus $\left(10^{3}\right.$ to $\left.10^{5} \mathrm{pfu} / \mathrm{mL}\right)$ with a detection limit of $10^{3} \mathrm{pfu} / \mathrm{mL}$ (1.3\%) (Fig. 12d). This free-standing graphene film can be suitably modified to be applied as flexible immunobiosensor devices in food safety measurement, clinical analysis, and environmental monitoring [114].

Recently, Kshama et al. fabricated an immunosensor based on graphene coated on a flexible PI substrate in the IDE pattern for the detection of cytokines (interleukin $10(1 \mathrm{~L}-10)$ and interferon-gamma (IFN- $\gamma)$ ). In this work, the GO/PI hybrid was fabricated through aerosol jet printing, as shown in Fig. 13a. The interdigitated electrodes with a high resolution of $40 \mu \mathrm{m}$ line widths were printed using the graphenenitrocellulose ink on a flexible Kapton (polyimide) substrate in the IDE pattern. They reported the first AJP flexible graphene IDE for the electrochemical immunosensing. The IDEs were thermally annealed in $\mathrm{CO}_{2}$ to generate highly reactive oxygen groups such as hydroxyl and carboxyl groups on the graphene surface, which enable the covalent binding of the appropriate antibody on the functionalized graphene surface for the monitoring of cytokines while maintaining the highly oriented structure of graphite underneath. The detection of desired antigens using the fabricated sensor was performed by monitoring the charge transfer resistance as a function of antigen concentration in bovine serum, and a higher sensitivity was observed for the sensing of IFN- $\gamma$ (Fig. 13c) and IL-10 (Fig. 13b) in a wide range of $0.1-5 \mathrm{ng} / \mathrm{mL}$ and $0.1-$ $2 \mathrm{ng} / \mathrm{mL}$ respectively. The resultant graphene-based immunosensors can detect cytokines in bovine implant serum with higher selectivity and a low detection limit. A low detection limit was observed at $25 \mathrm{pg} / \mathrm{mL}$ and $46 \mathrm{pg} / \mathrm{mL}$ of IFN- $\gamma$ and IL-10, respectively. The high selectivity is observed due

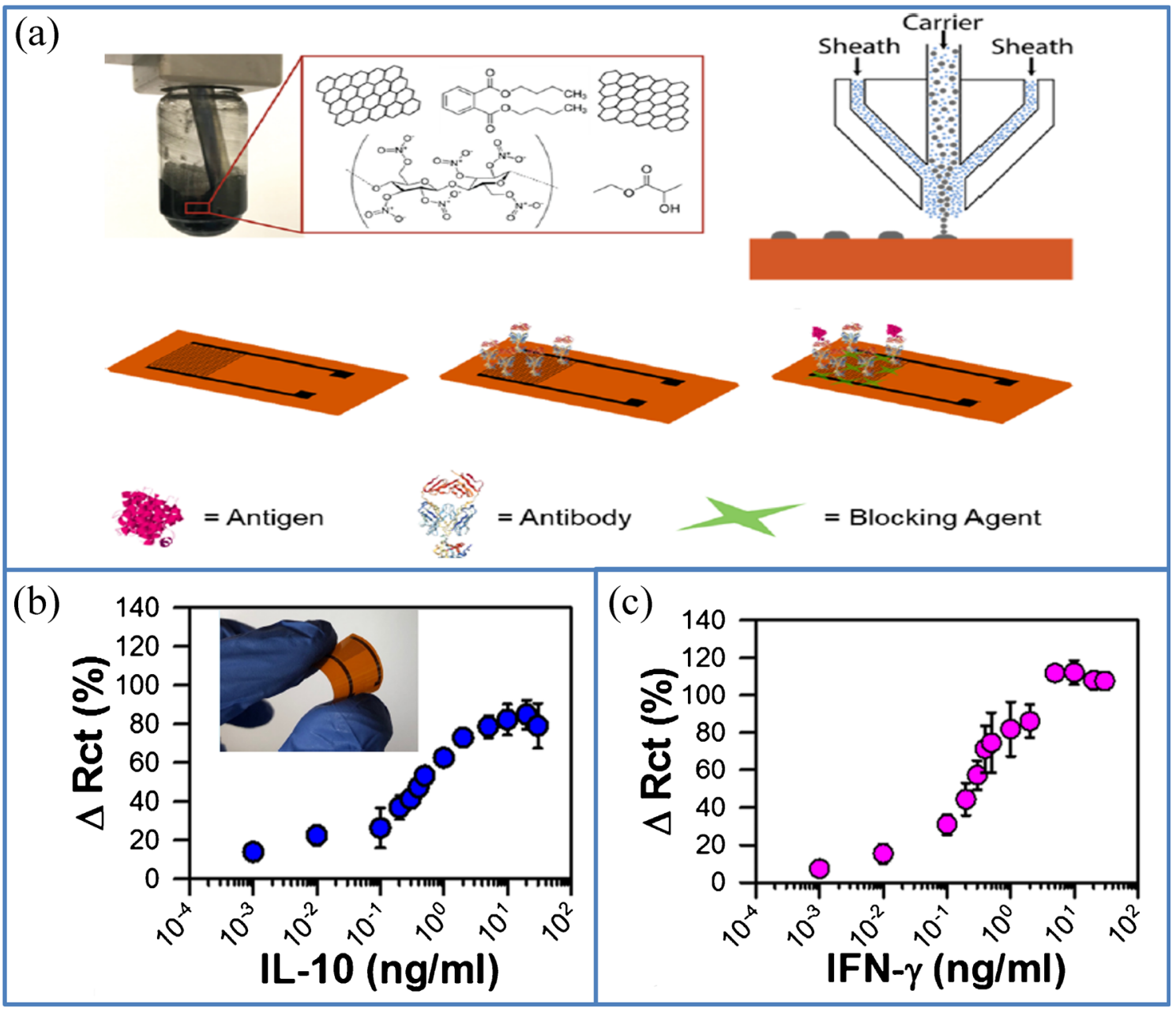

Fig. 13 a Schematic image of the fabrication and functionalization of the AJP graphene in IDE pattern, which includes the formulation of graphene ink for aerosol printing, printing of graphene ink on PI substrate, immobilization of antibodies on the functionalized graphene surface, and the covering of a surface with blocking agent to prevent nonspecific adsorption during biosensing. The detection of $\mathbf{b}$ IL-10 and $\mathbf{c}$

IFN- $\gamma$ using the AJP graphene IDE sensors, the photograph of the bendable graphene IDE sensor, is shown in the inset of c (source: reprinted from Parate K, Rangnekar $S$ V, Jing D, Mendivelso-P D L, Ding S, Secor $E B$, et al. Aerosol-jet-printed graphene immunosensor for label-free cytokine monitoring in serum. ACS Appl Mater Interfaces. 2020;12:8592603, Copyright@2020,ACS) 
to a minimum cross-reaction of antibodies with each other and similar IL-6 cytokines. The flexibility of the functionalized IDE was investigated by monitoring the electrochemical impedance and electrical characteristics as a function of bending cycles; hence, it could be executed in an implantable or wearable design such as a tiny hypodermic bovine implant. Besides, the variation in electrical resistance of the device due to mechanical flexing cycles is almost negligible $(<1 \%)$. The photographs of the bending test setup using IL10 functionalized biosensor (AJP graphene IDE) are shown in the inset of Fig. 13b. The flexible biosensors exhibited a stable response even after 250 bending cycles ( $\Phi$ (curvature) $-5 \mathrm{~mm}$ ) with a response time of $33 \mathrm{~min}$. The advantage of the AJP graphene immunosensor is their ability to detect the biomarkers in the range of $0.1-10 \mathrm{ng} / \mathrm{mL}$, which is suitable for the early monitoring of cytokine-related diseases such as paratuberculosis in humans and Johne's disease commonly found in cattle's [115]. This point-of-care diagnostic sensor will minimize the difficulties in handling, transportation time, and sample degradation and storage for the rapid and accurate treatment of diseases. The rapidly functionalized surface of graphene and the generality of antibody functionalization custom allow the selective sensing of interested analytes without the requirement of any sample pretreatment. This flexible graphene circuits are applicable for the sweat-based analysis of ion and protein, for heavy metal monitoring through skinintegrated devices, and in solar cells, organic LEDs, and pressure sensors [116-118].

The fabrication of biosensors based on TMDs such as $\mathrm{WSe}_{2}$ and $\mathrm{MoS}_{2}$ has been widely reported because of characteristic semiconducting and electric charge effect property. Specifically, the $\mathrm{MoS}_{2}$-based biosensor has some unique advantages such as biocompatibility, high surface area, and electrochemical property [50]. Nevertheless, the flexible biosensors comprised of nanomaterials have been fabricated through a complicated procedure. The PET can be explored as an excellent flexible substrate for the fabrication of flexible immunosensor because of its low cost, chemical resistance, dimensional stability, and good thermal conductivity compared to the flexible PI substrate [119]. Various diseases such as influenza, severe acute respiratory syndrome (SARS), and acquired immune deficiency syndrome (AIDS) are created by tiny microbial agents known as viruses. Among the fatal diseases caused by viruses, human immunodeficiency virus (HIV) has gotten intensive attention since it gives rise to AIDS, which affects the human immune system, ultimately leading to death. HIV is known to have a long incubation period with an ability to transmit throughout this incubation period, and the precise diagnosis is a significant problem in clinical assays [120]. The HIV surface is comprised of a glycoprotein envelope, which contains non-covalently bonded GP120 (gp120) glycoprotein and GP41 (gp41) glycoprotein. Various methods such as polymerase chain reaction (PCR) and ELISA have been explored for HIV detection. Nevertheless, these methods are restricted due to less sensitivity, time consumption, and intricate sample treatment. To conquer these restrictions, electrochemical immunosensors have emerged for the highly sensitive and instantaneous detection of HIV. To account for all these points, Minkyu et al. presented a highly sensitive and flexible immunosensor using $\mathrm{Au} /$ $\mathrm{MoS}_{2} / \mathrm{Au}$ nanolayer deposited on a flexible PET substrate to detect the HIV protein gp120 using polyvinyl pyrrolidine (PVP)-modified $\mathrm{MoS}_{2}$ NPs deposited on the PET substrate. This PVP modification of $\mathrm{MoS}_{2}$ nanoparticles was done to obstruct the agglomeration of $\mathrm{MoS}_{2}$ nanoparticles to ease their dispersion on the flexible PET substrate. This particular immunosensor was made by the sputter coating of $\mathrm{Au}$ on the PET substrate, followed by the sputter coating of $\mathrm{MoS}_{2}$ nanoparticles on $\mathrm{Au}$, which is then coated again using Au NPs. The nanolayer of $\mathrm{Au} / \mathrm{MoS}_{2} / \mathrm{Au}$ is modified with cysteamine (Cys) to bind with the gp120 antibody, which is immobilized to the flexible electrode surface (Fig. 14a). The SEM image of the sputter-coated $\mathrm{Au} / \mathrm{MoS}_{2} / \mathrm{Au}$ on the PET substrate is given in Fig. 14b. As illustrated in Fig. 14 c, the selective detection of the gp120 antigen was performed by square wave voltammetry. The fabricated electrochemical immunosensor showed a highly sensitive response to gp120 antigen $(0.1 \mathrm{pg} / \mathrm{mL}$ to $10 \mathrm{ng} / \mathrm{mL}$ ) in human serum with a detection limit of $0.066 \mathrm{pg} / \mathrm{mL}$ compared to other antigens such as $\mathrm{Mb}$, Trx, PSA, and $\mathrm{Hb}$ (Fig. 14d). A micro-fatigue tester was used to investigate the flexibility of the immunosensor. Both the $\mathrm{Au} /$ $\mathrm{MoS}_{2} / \mathrm{Au}$ nanolayer on PET and Au sputter-coated PET showed good flexibility compared to the conventional gold electrodes as illustrated in Fig. 14e. The fabricated sensor exhibited magnificent flexibility and flexure extension (1.54 $\mathrm{mm}$ ) compared to other conventional Au electrodes and stable detection even after bending. Besides, the proposed immunosensor can be commercialized as a flexible sensing platform to design highly efficient wearable sensor devices for the treatment of HIV infected patients [121]. Table 2 shows the performance of flexible polymer-based electrochemical immunosensors based on $2 \mathrm{D}$ materials so far reported.

\section{Paper-based immunosensors}

For the first time, Wu et al. reported the integration of a signal amplification strategy into a paper-based microfluidic device for the highly sensitive multiplexed detection of various cancer biomarkers, including alfa-fetoprotein, cancer antigen 125 (CA125), carbohydrate antigen 153 (CA153) and carcinoembryonic antigen (CEA). The signal amplification strategy was realized by the modification of the immune device surface using graphene to promote the electron transfer and thereby magnified the detection signal. Further amplification was attained through the reduction in physical adsorption 
Table 2 Overview of flexible electrochemical immunosensors based on two-dimensional materials for clinical analysis

\begin{tabular}{llllc}
\hline Electrode & Analyte & Linear range & Limit of detection & References \\
\hline AJP graphene IDE/PI & IFN- $\gamma$ & $0.1-5 \mathrm{ng} / \mathrm{mL}$ & $25 \mathrm{pg} / \mathrm{mL}$ & {$[115]$} \\
& IL-10 & $0.1-2 \mathrm{ng} / \mathrm{mL}$ & $46 \mathrm{pg} / \mathrm{mL}$ & \\
AJP graphene IDE/PI & Histamine & $56.25 \mu \mathrm{M}-1.8 \mathrm{mM}$ & $30.7 \mu \mathrm{M}$ & {$[113]$} \\
GO film & Rotavirus & $10^{3}-10^{5} \mathrm{pfu} / \mathrm{mL}$ & $10^{3} \mathrm{pfu} / \mathrm{mL}$ & {$[114]$} \\
$\mathrm{Au} / \mathrm{MoS}_{2} / \mathrm{Au} / \mathrm{PET}$ & gp 120 & $0.1 \mathrm{pg} / \mathrm{mL}-10 \mathrm{mg} / \mathrm{mL}$ & $0.066 \mathrm{pg} / \mathrm{mL}$ & {$[121]$} \\
\hline
\end{tabular}

using silica NPs with immobilization of horseradish peroxidase (HRP) and antibody. The fabrication of the proposed sensor system consists of various steps, such as the impregnation of photoresist on a Whatman chromatography paper and spin coating to make uniform distribution over the paper (Fig. 15a). The area of filter paper coated by the

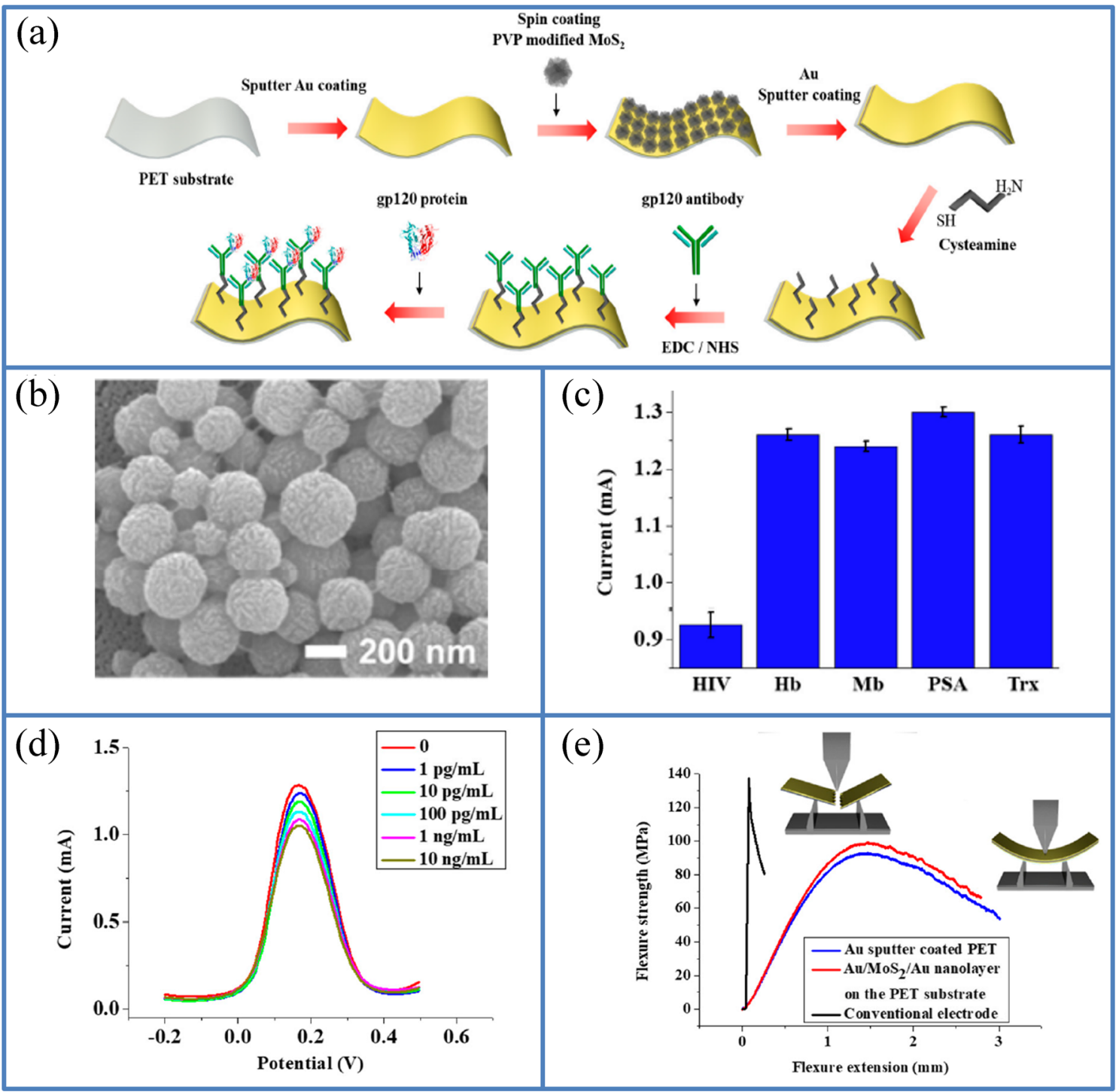

Fig. 14 a Schematic representation of the fabrication of flexible immunosensor (gp120 Ab/Cys/Au/MoS $/ 2 \mathrm{Au}$ ) nanolayer deposited on the PET substrate through spin coating for the detection of gp120 antigen. b SEM image of the $\mathrm{Au} / \mathrm{MoS}_{2} / \mathrm{Au}$ nanolayer on PET. c Selectivity bar diagram of gp120 (Ab)/Cys/Au/ $\mathrm{MoS}_{2} / \mathrm{Au}$ nanolayer on flexible PET substrate to different types of antigens and proteins such as $\mathrm{Hb}, \mathrm{Mb}$, PSA, and Trx in PBS solution. d Square wave voltammetry

(SWV) results from the detection of gp120 antigen in human serum with concentration range between 0.1 and $10 \mathrm{pg} / \mathrm{mL}$. e Flexure plots of $\mathrm{Au}$ sputter-coated PET, Au/MoS $/ 2 \mathrm{Au}$ on the flexible PET substrate, conventional electrode (source: reprinted from Shin M, Yoon J, Yi C, Lee T, Choi $J W$. Flexible HIV-1 biosensor based on the au/MoS 2 nanoparticles/au nanolayer on the PET substrate. Nanomaterials. 2019; 9:1-12) 


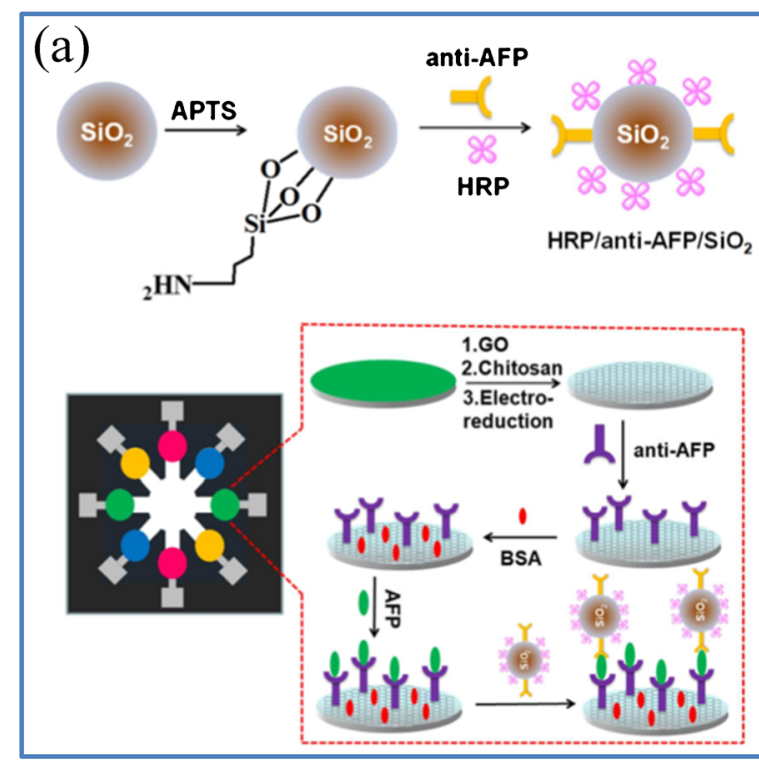

Fig. 15 a Schematic representation for the fabrication of nanobioprobes via the coimmobilization of antibody and HRP on to monodispersed silica NPs and assay procedure utilized to synthesize microfluidic paper-based electrochemical immunodevice (AFP was given as an example) and $\mathbf{b}$ electrocatalytic currents detected for the different antigens on

photoresist was hydrophobic, and the remaining region was kept as hydrophilic. The counter electrode and reference electrodes were constructed by the screen printing of $\mathrm{Ag} / \mathrm{AgCl}$ ink and carbon ink, respectively, on the predetermined zones of paper B. Similarly, the particular area of paper A was screen printed by eight working electrode zones with carbon ink. Then the electrochemical work station was connected to the paper-based microfluidic device through conductive pads made of silver paints. Finally, graphene was deposited on the working electrode and the antibodies were immobilized on the corresponding working electrodes. They synthesized four kinds of nanobioprobes including HRP/anticarcinoembryonic antigen (CEA)/ $/ \mathrm{SiO}_{2}, \mathrm{HRP} /$ anti- alphafetoprotein (AFP)/SiO ${ }_{2}, \mathrm{HRP} /$ anti-cancer antigen (CA)-153/ $\mathrm{SiO}_{2}$, and $\mathrm{HRP} / \mathrm{CA}-125 / \mathrm{SiO}_{2}$, which comprised silica NPs with antibodies and HRP alpha-fetoprotein co-immobilized on the probe surface against the corresponding analyte (Fig. 15a). The detection signal was produced in the presence of $\mathrm{H}_{2} \mathrm{O}_{2}$ and $\mathrm{O}$-phenylenediamine. The analytical performance of the fabricated immunodevice to the biomarkers was monitored through differential pulse voltammetry (DPV), the electrocatalytic currents detected for the different antigens on the different working electrodes, as illustrated in Fig. 15b. Experimental results confirmed that with the increase in concentrations of AFP, CA125, CA153, and CEA, well correlations between the DPV current response and the concentrations of tumor biomarkers with similar dynamic working ranges $\left(0.001-100 \mathrm{ng} \mathrm{mL}^{-1}, 0.001-100 \mathrm{ng} \mathrm{mL}^{-1}\right.$, $0.005-100 \mathrm{ng} \mathrm{mL}^{-1}, 0.005-100 \mathrm{ng} \mathrm{mL}^{-1}$,), which analogs to the level of the biomarker that was found naturally in human

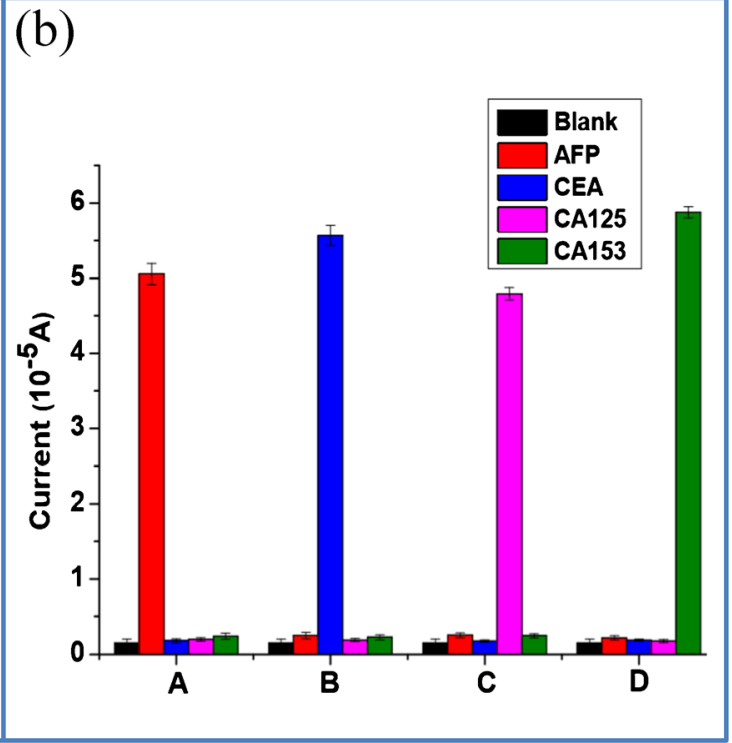

the different working electrodes (source: reprinted from $W u Y$, Xue $P$, Kang Y, Hui KM. Paper-based microfluidic electrochemical immunodevice integrated with nanobioprobes onto graphene film for ultrasensitive multiplexed detection of cancer biomarkers. Anal Chem. 2013;85:8661-8,copyright@2013,ACS)

serum and plasma samples. The limit of detections for the four cancer biomarkers was observed to be at $0.001 \mathrm{ng} \mathrm{mL}^{-1}$, $0.001 \mathrm{ng} \mathrm{mL} L^{-1}, 0.005 \mathrm{ng} \mathrm{mL}^{-1}$, and $0.005 \mathrm{ng} \mathrm{mL}^{-1}$, much lower compared to the previously reported studies. This proposed system offers several advantages such as disposability, simplicity, portability, and cost-effective paper strips with the ability for multifold analyte detection [122]. In recent years, lung cancer is known as a malignant tumor with the highest mortality rate, whose proper cure has not yet been possible, and even now, there is a perennial rising trend. Endeavoring for early diagnosis, premature detection, and premature treatment are the main standards to reduce the mortality rate of lung cancer. In clinical assays, the blood serum of cancer patients is characterized by higher levels of tumor markers. Certain types of carcinomas such as gastric, pancreatic, colorectal, and cervical could be diagnosed using carcinoembryonic antigen (CEA), a widely used tumor marker in the clinical analysis [123]. Meantime, the levels of CEA in blood serums are connected to the various stages of the tumor; hence, it can be explored as a tumor marker to directly monitor therapeutic effects, recovery, and metastasis [124]. So, highly sensitive and precise clinical assays for detecting CEA with a low level in biological samples are required for effective premature diagnosis and treatment of cancer [125]. There are various assays, such as chemiluminescence assay, radioimmunoassay, and so forth, to detect CEA in tumor patients [126]. However, these assays are sensitive, they normally needed large instruments, and these kinds of assay processes are more difficult and time-consuming. Since the foundation of the ELISA test, it has become a commonly used 
technique for cancer diagnosis, but the need for highly expensive tool kits limits ELISA's practicality for point-of-caretesting (POCT) [127]. On the contrary, label-free electrochemical immune assay has become the most prominent research hot spots to achieve the requirements of POCT. The combination of label-free electrochemical technique and nanotechnology constitutes an important approach for the cancer diagnosis, which permits highly sensitive, cost-effective, fast and highly specific electrochemical nano-devices for the monitoring of tumor biomarkers and dispenses superior precision in cancer diagnosis. Wang et al. fabricated a novel label-free electrochemical immunosensor on a microfluidic paper device utilizing a screen-printed carbon working electrode (SPWE) for the detection of CEA. The SPWE was modified using $\mathrm{NH}_{2}-\mathrm{G}$ (amino-functional graphene)/Thi (thionine)/Au NP (gold nanoparticle) nanocomposites coating for the immobilization of anti-CEA and improvement in the detection sensitivity (Fig. 16a). Graphene has been known as a promising material for the fabrication of electrochemical immunosensor due to is unique physical and chemical properties. They used two pieces of Whatman No. 1 cellulose filter paper that is selectively patterned to fabricate the microfluidic paper-based devices. In the initial step, the immunodevice structure was designed using Adobe Illustrator CS5 on the computer. A three-electrode system is used for the detection of CEA, which includes the counter electrode, working electrode, and reference electrode. The design of the patterned working electrode, a reference electrode, and the counter

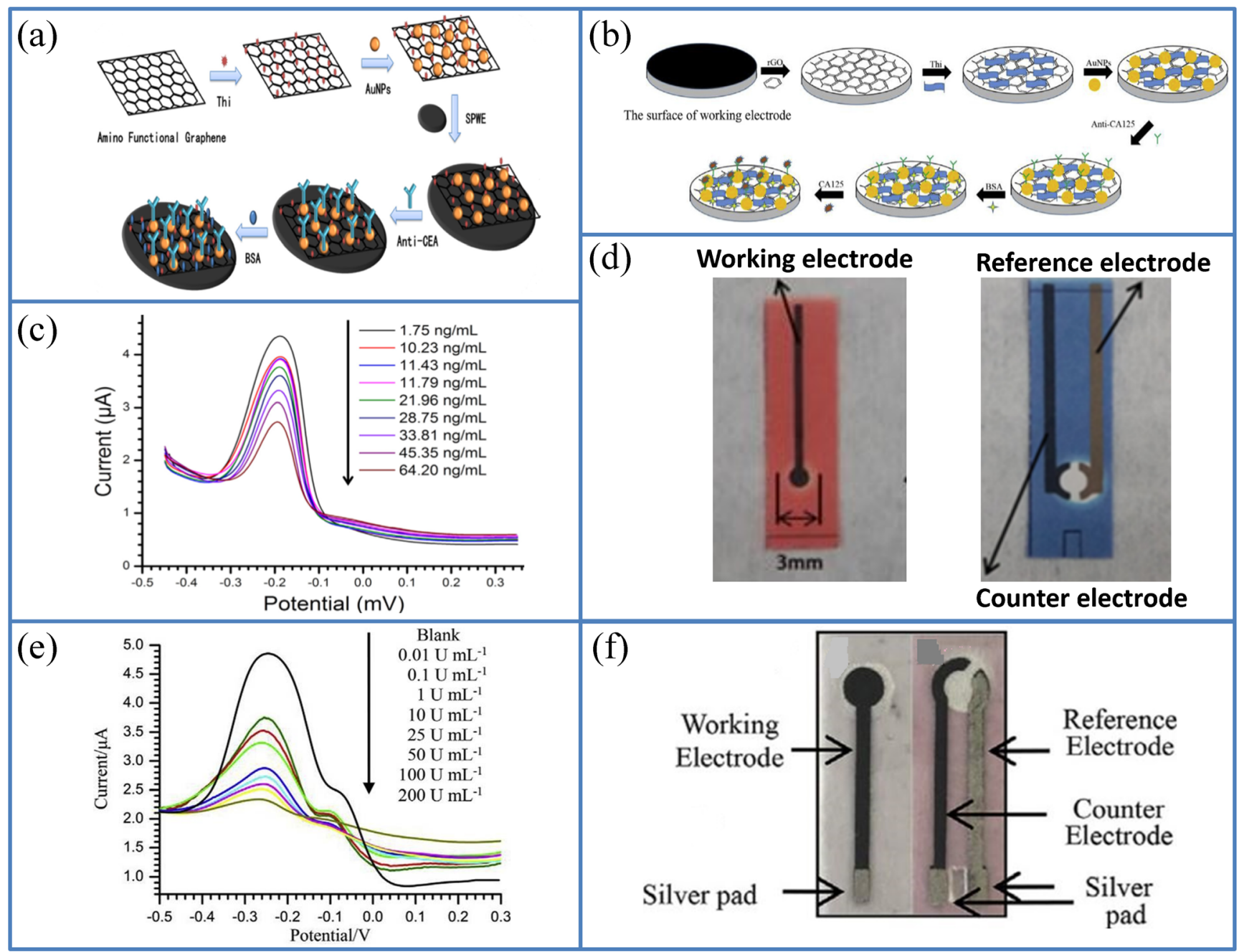

Fig. 16 Schematic representation for the preparation of $\mathrm{NH}_{2}-\mathrm{G} / \mathrm{Thi} /$ AuNPs nanocomposites and immune assay protocol for a antigen CEA and b CA125; c DPV responses of immunosensor for the detection of CEA in clinical serum sample with different concentrations; d photographs of the WE, REF, and counter electrode after cutting (source: reprinted from Wang Y, Xu H, Luo J, Liu J, Wang L, Fan YA. novel label-free microfluidic paper-based immunosensor for highly sensitive electrochemical detection of carcinoembryonic antigen. Biosens
Bioelectron.2016;83:319-26, with permission from Elsevier). e DPV responses of immunosensor for the detection of CA 125 with different concentrations. f Photograph of immunosensor with silver pad for the detection of CA 125 (source: reprinted from Fan Y, Shi S, Ma J, Guo $Y$. A paper-based electrochemical immunosensor with reduced graphene oxide/thionine/gold nanoparticles nanocomposites modification for the detection of cancer antigen 125. Biosens Bioelectron. 2019;135:1-7, with permission from Elsevier) 
electrode is in Fig. 16d; the pattern is designed given the operational convenience of the immunosensor device. Besides, they adopted a second approach in which the immunosensor device was patterned and then fabricated via wax printing on a cellulose paper. The porous structure of the paper allows the penetration of wax through the paper to form the insulating and hydrophobic patterns; meanwhile, the unpatterned area of the paper contains a sample zone and auxiliary zone maintains its flexibility, porous structure, and hydrophilicity. After this, the wax-coated paper sheet was organized for the electrodes screen printing on the corresponding zone. During the third approach, they explored a fast and cost-effective screen printing technique for the printing of three electrodes on the corresponding tab. As described in Fig. 16d, the sample tab includes the carbon working electrode (Acheson carbon ink) while the auxiliary tab contains both counter electrode (Acheson carbon ink) and a reference electrode ( $\mathrm{Ag} / \mathrm{AgCl}$ ink). Eventually, the paper sheet was made into the origami device for the additional modifications. In the final stage, the two pads were blended together using double-sided adhesive tapes. They fabricated a portable electrochemical measuring instrument and then inserted an immunosensor device at the interface which can directly be connected to the instrument, as shown in inset of Fig. 16c. The basis of the immunosensor detection was depending on the fact that the reduction in the current response of thionine was proportional to the corresponding antigen (CEA) concentration due to the formation of hydrophobic immunocomplex by the interaction of CEA antibody and antigen. The faster and simpler detection of CEA was done through a new label-free electrochemical method, which ignores the labeling of either antibodies or antigens. These fully functionalized $\mu$ PADs have emerged as an effective tool for CEA detection, which can be used for point-of-care testing. The fabricated immunosensor showed high sensitivity to CEA in a range of $50 \mathrm{pg} \mathrm{mL}^{-1}$ to $500 \mathrm{ng} \mathrm{mL}^{-1}$ with a low detection limit of $10 \mathrm{pg} \mathrm{mL}^{-1}$. Moreover, the sensor could be explored to detect large numbers of clinical serum samples and relative errors were calculated for the reference concentrations and measured values. The concentration of clinical serum samples is in the range between 1.75 and $64.20 \mathrm{ng} \mathrm{mL}^{-1}$, as shown in Fig. 16c. The current of DPV response is different from the standard CEA solutions due to other chemical substances, which could affect the specific antigen-antibody interactions. The sensitivity of the fabricated system towards the standard CEA solutions was about 1.04 times higher than that of clinical serum samples. The results confirmed that the fabricated electrochemical immunosensor can be explored as an emerging platform for highly sensitive, cost-effective, and point-of-care diagnostics [127]. Similarly, Fan et al. presented a paper-based electrochemical immunosensor for the detection of CA125 antigen using a screen printing method. The nanocomposites based on $\mathrm{r}-\mathrm{GO} / \mathrm{Thi} / \mathrm{Au} \mathrm{NPs}$ were combined and then coated on the working electrode of the sensor for the immobilization of the corresponding antibody (anti-CA125) and amplification of the detection signal (Fig. 16b). Graphene is a potential nanomaterial in the biomedical domain owing to biocompatibility, conductivity, and high surface area. Au metal nanoparticles are a good choice for the signal amplification in electrochemical measurement due to its high surface area and better electrical properties. Thionine was employed as an electroactive material for producing redox current response at the time of testing. The nanocomposites of r-GO/Thi was formed through the $\pi-\pi$ stacking interaction, in which the benzene ring of thionine molecules can non-covalently bind to r-GO. Then Au NPs were immobilized on the r-GO/Thi through the interaction of its amino groups with the Au NPs (Fig. 16b). The detection principle of the immunosensor relied on the fact that the decrease in current response of thionine was proportional to the corresponding CA125 antigen concentration owing to the formation of immunocomplex by the interaction of CA125 antibody and antigen, as shown in Fig. 16e. The analytical performance of the proposed immunosensor is shown in Fig. 16e; the decrease in current response corresponding to the increase in CA125 antigen concentration was observed due to the immunocomplex formation between the CA125 antibody and antigen. The reason might be because of the complex, which creates a barrier in the electron transfer towards the electrode surface by blocking the electron transfer tunnel. The photograph of the single electrochemical immunosensor fabricated on a cellulose paper is shown in Fig. 16f. The experimental results of immunoassay showed the detection of standard CA125 solutions with linear working ranges of 0.1 to $200 \mathrm{U} \mathrm{mL}^{-1}$, and the limit of detections for standard CA125 is $0.01 \mathrm{U} \mathrm{mL}^{-1}(S / N=3)$. The proposed electrochemical immunosensor was used to monitor clinical serum samples; the results showed a justifiable agreement with the classic EISA test, and the relative error was observed to be less than $8.05 \%$ during clinical diagnosis. The fabricated immunosensor showed good electrochemical response with high precision, stability, accuracy, and reliability. This proposed sensor can emerge as a potential tumor biomarker for the point-of-care diagnosis of other tumors [128]. Ruecha et al. recently developed a novel label-free electrochemical impedance immunosensor coupled with a paper-based microfluidic device for the highly sensitive detection of human interferon $\gamma$ (IFN- $\gamma$ ), which is a cytokine generated mainly by natural killer cells and $\mathrm{T}$ lymphocytes with regard to antigen stimulation; it can be used as a biomarker during tuberculosis diagnosis. They used cyclic voltammetry to deposit polyaniline on the paper electrode with screenprinted graphene. This modified electrode allows high surface area for the immobilization of antibody and high electrochemical conductivity which is suitable for enhancing the sensitivity. The synthesized electrochemical sensor was showed enhanced sensing performance to human IFN- $\gamma$ in a range of 5- 
$1000 \mathrm{pg} \mathrm{mL}^{-1}$ with a low detection limit of $3.4 \mathrm{pg} \mathrm{mL}^{-1}$. The PANI-graphene-modified electrode exhibited a sensitivity of about 31 times higher than that of PANI-modified electrodes. This enhanced performance was observed due to the incorporation of graphene with PANI into the sensor system, which offered highly functional amino groups on PANI and high electrochemical sensitivity. Graphene accelerated the electron transfer kinetics of the device and improved the electrochemical conductivity of the sensor. This PANI-G electrode was effectively applied for the detection of IFN- $\gamma$ in human serum samples with better reproducibility. Besides, the fabricated sensor system offers various advantages such as the requirement of low-volume samples, cost-effectiveness, disposability, and fast analysis compared to classic traditional methods, allowing this sensing platform to be explored as an effective analytical tool for antigen IFN- $\gamma$ screening [129].

Iron is an essential mineral in the human body, plays a pivotal role in the functioning of different organ systems, including the immune system, blood circulation, and metabolism. The WHO has been reported iron deficiency as a global health issue, hence additional care is needed at the time of blood donation to ensure the safety of recipients, and a useful screening test is crucial and necessary in clinical diagnosis $[130,131]$. Ferritin is an iron-containing protein responsible for the metabolic process of $\mathrm{Fe}$ stored in the blood cells, which can be explored as a pivotal biomarker to monitor the riskiness of ironic deficiency anemia [132]. In males and females, the normal concentrations of ferritin should be in a range of 30 $300 \mathrm{ng} \mathrm{mL}^{-1}$ and $15-200 \mathrm{ng} \mathrm{mL}^{-1}$, respectively [133]. The fluctuation in the levels of ferritin can lead to several health issues such as infection, obesity, cancer, ironic deficiency, hemochromatosis, and nephritis [134]. Electrochemical techniques meet the requirements of point-of-care devices due to their high sensitivity, fastness, portability, and the capability to execute real-time analysis [135]. The electrodes can be miniaturized into an accessible device due to the requirement of low sample volume. Electrochemical monitoring of crucial biomarkers depending on sandwich-type format utilizing different labeling with bio- and/or nanomaterials has been studied. Boonkaew et al. fabricated a highly sensitive label-free paper-based electrochemical immunosensor using an inkjetprinted working electrode for the detection of ferritin. The pattern of the device was traditionally designed on a filter paper for the successful realization of immunosensor. The fabrication of ePAD includes various steps such as printing of device pattern on the Whatman no. 1 filter paper via wax printing to realize hydrophobic and hydrophilic areas and inhouse screen printing of three-electrode systems on the patterned paper (Fig. 17a). The screen printing of graphene inks on the hydrophilic portion of the board was done to form the working electrode and counter electrode, the printing of Ag/ $\mathrm{AgCl}$ inks to create the conductive pads and reference electrode. The inkjet printing technique was employed to modify the working electrode surface by the drop-casting of the graphene oxide solution. Then the electrode was activated via 1-ethyl-3-(3-dimethylaminopropyl)carbodiimide (EDC)/ N-hydroxysulfosuccinimide (sulfo-NHS) chemistry for the covalent immobilization of anti-ferritin antibodies on the amine-functionalized ester surface of the electrode (Fig. 17b). The electrochemical current response of the bovine serum albumin (BSA)/anti-ferritin (anti-FTH)/graphene oxide (GO)/screen-printed graphene electrode (SPGE) electrode was monitored with and without ferritin in Fig. $17 \mathrm{~b}$ to confirm detection of ferritin. The reduction in the signal response shows the formation of the antigen-antibody barrier on the electrode surface that impedes the redox couple diffusion to the electrode. On the contrary, there was no significant change in current response observed in the absence of biomarkers. The selectivity of the proposed sensor was confirmed through the testing of immunosensor with various interfering species that can be usually found in a real sample. The experimental results of immunoassay showed the detection of ferritin with linear working ranges of 1 to $1000 \mathrm{ng} \mathrm{mL}^{-1}$; the limit of detection was observed to be $0.19 \mathrm{ng} \mathrm{mL}^{-1}(S / N=3)$. The proposed handmade paper-based sensor showed high sensitivity, selectivity, and reproducibility; it can be utilized as an alternative device for the anemia diagnosis [136].

E. coli constitute a large group of rod-shaped gram-negative bacteria usually found in the intestinal areas of animals and humans. But particular strains of $E$. coli can result in urinary tract infection (UTI) and food poisoning. It is reported that $90 \%$ of urinary infections are due to the growth of E. coli. This situation demands the development of efficient and costeffective sensors to detect $E$. coli bacteria. The E. coli O157:H7 bacterium is regarded as a hazardous foodborne pathogen that can cause hemolytic-uremic syndrome (HUS), hemorrhagic colitis, and other complicated diseases. In literature, many reports are available on immunosensors for the electrochemical detection of the E. coli bacterium. Still, fewer reports are available on flexible electrochemical immunosensors based on two-dimensional materials for the selective detection of bacteria. Several reports are available on the development of sensors on hard substrates to detect E. coli, utilizing functionalized graphene or CNT channel. Akhavan et al. had done several experiments to explain the sensing response of graphene and graphene oxide. They reported that the GOS (graphene oxide nanosheet) with oxygenated functional groups could trap the bacteria better than graphene nanosheet (GS), which is characterized by reduced functional groups [137]. In many reports, a linker/antibody was functionalized on graphene for the selective detection of E. coli bacteria.

There are various sensors available for the detection of E. coli bacteria; among them, impedimetric immunosensors got a huge attraction for detecting E. coli 0157:H7 because of their fast, label-free, and portable characteristics. But large- 


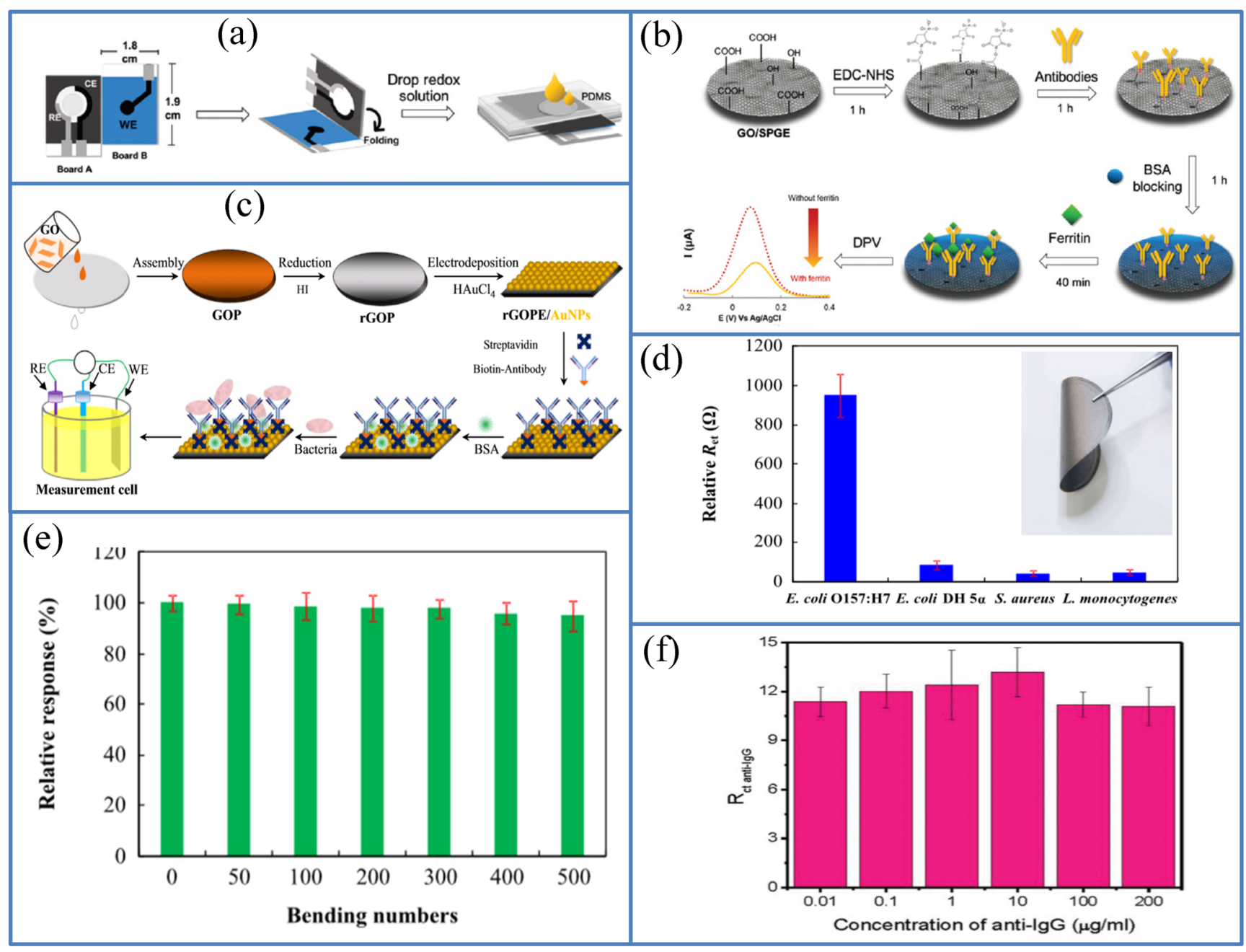

Fig. 17 Schematic demonstration for a the design and fabrication of ePAD, b overall preparation of impedimetric immunosensor including immobilization step for the detection of ferritin and DPV response of sensor with and without ferritin. (Source: reprinted from Boonkaew $S$, Teengam P, Jampasa S, Rengpipat S, Siangproh W, Chailapakul O. Cost-effective paper-based electrochemical immunosensor using a label-free assay for sensitive detection of ferritin. Analyst. 2020;145:5019-5026, with permission from RSC). b The complete fabrication process including rGOP fabrication, AuNP deposition, and modification using biotin-streptavidin, antibody immobilization, and E. coli detection of the rGOP-based impedimetricimmunosensor, $\mathbf{d}$ plot for the relative response of $E$. coli against various bacteria with a

scale applications are often limited due to the highly expensive gold electrodes. Graphene paper was first reported in 2007 and received a lot of attention for the fabrication of sensor devices due to good conductivity, high flexibility, and light weight. Graphene paper as a derivative of graphene or GO-supported building blocks was presented by Ruoff et al. in 2007 [138]. The development of new-generation flexible electrodes with sufficient physiochemical properties is getting promoted by the use of graphene paper owing to their characteristic mechanical property, variable functionality, and tunable conductivity. It is possible to control the periodic arrangement of graphene nanosheets while making into two- photograph of rGOP in the inset, e the impedance response of rGOPbased immunosensor $\mathrm{v} / \mathrm{s}$ bending numbers for the detection of $E$. coli 0157:H7 $\left(1.5 \times 10^{5} \mathrm{cfu} \mathrm{mL}^{-1}\right)$. (Source: reprinted from Wang Y, Ping $J$, Ye Z, Wu J, Ying Y. Impedimetric immunosensor based on gold nanoparticles modified graphene paper for label-free detection of Escherichia coli O157: H7. Biosens Bioelectron. 2013;49:492-498, with permission from Elsevier). f Impedimetric response of TR-GO modified DEP electrode surface for the detection of $\mathrm{IgG}$ (signal as $\mathrm{R}_{\mathrm{ct}}$ anti-IgG) (source: reprinted from Loo AH, Bonanni A, Ambrosi A, Poh HL, Pumera M (2012) Impedimetric immunoglobulin $G$ immunosensor based on chemically modified graphenes. Nanoscale 4:921-925, with permiion from $R S C)$

dimensional graphene films/paper membranes via hydrophobic effect, ionic interaction, $\pi-\pi$ stacking, and/or hydrogen bonding, and generate new properties resulting from its characteristic structure. Wang et al. presented cost-effective and robust electrochemical impedimetric immunosensors based on Au NP-modified free-standing graphene paper for the highly sensitive detection of E. coli $0157: \mathrm{H} 7$, where the graphene paper was prepared through the chemical reduction of graphene oxide paper constructed from the vacuum filtration method. The electrodeposition approach was explored for the growth of Au NPs on the graphene paper surface. Au nanoparticles were used to ensure an appropriate environment 
for the immobilization of biomolecules and promote the electron transfer between the electrode and the immobilized biomolecules. A biotin-streptavidin system was used for the immobilization of antibodies such as anti- E. coli $0157: \mathrm{H} 7$ on the surface of the paper electrode. A schematic illustration of the complete fabrication process of the proposed impedimetric immunosensor is given in Fig. 17c. The photograph of bendable r-GOP (reduced graphene oxide paper) with a shiny metallic luster is given in the inset of Fig. 17d. The E. coli bacteria trapped on the electrode surface were monitored via electrochemical impedance spectroscopy. The real matrix including cucumber and ground beef, the most common contaminated foods, was used for the monitoring of $E$. coli bacteria to confirm the feasibility of the rGOP-based immunosensor and the low detection limit was found to be $1.5 \times 10^{4} \mathrm{cfu} \mathrm{mL}^{-1}$ and $1.5 \times 10^{3} \mathrm{cfu} \mathrm{mL}^{-1}$ for ground beef samples and cucumber samples, respectively. The experimental results of immunoassay showed the detection of $E$. coli with linear working ranges of $1.5 \times 10^{2}$ to $1.5 \times 10^{7} \mathrm{cfu} \mathrm{mL}^{-1}$; the limit of detection was observed to be $1.5 \times 10^{2} \mathrm{cfu} \mathrm{mL}^{-1}(S / N=3)$ with excellent specificity. The system specificity for the detection of $E$. coli 0157:H7 was monitored by testing with other microorganisms, such as S. aureus, E. coli DH $5 \mathrm{a}$, and L. monocytogenes, as shown in Fig. 17d. No marked interference was observed from these non-target bacteria $\left(10^{7} \mathrm{cfu} \mathrm{mL}^{-1}\right)$, suggesting a new feasible sensing platform based on flexible rGOP for the specific detection of E. coli 0157:H7. The proposed sensor was tested for flexibility, and the results confirmed the high tolerance level of the graphene paper-based sensing device to mechanical stress. At the same time, the impedance response of the sensor to $E$. coli $(1.5 \times$ $10^{2} \mathrm{cfu} \mathrm{mL}^{-1}$ ) decreased to less than $5 \%$ after a $90^{\circ}$ inward bending of the paper electrode for 500 bending cycles (Fig. 17e). The system could be used at least 24 times without any significant loss in sensing response. Flexibility is a crucial factor while developing the lab-on-a-chip devices and implantable immunodevices based on the paper substrate for the in vivo and in vitro analysis of real-life samples in the clinical field. The proposed sensor has provided a new insight into the fabrication of flexible immunosensors by adopting an advanced strategy of structural integration using biomolecules, graphene paper, and metal NPs for the sensing applications [79]. Recently, Loo et al. presented a label-free electrochemical impedimetric immunosensor to detect immunoglobulin $\mathrm{G}$ (IgG) using chemically modified graphene surfaces, including graphene oxide, graphite oxide, electrochemically reduced graphene oxide and thermally reduced graphene oxide. The anti-IgG corresponding to $\operatorname{IgG}$ was immobilized on the disposable electrochemical printed electrodes (DEP) modified with chemically modified graphene surfaces. The detection of IgG was based on the changes in impedance spectra due to the interaction of IgG towards the immobilized antiIgG, and the TR-GO gave the best performance for the detection of $\operatorname{IgG}$ with a linear range of detection from 0.3 to $7 \mu \mathrm{g} \mathrm{mL}^{-1}$; the LOD was about $10 \mu \mathrm{g} \mathrm{mL}^{-1}$ (Fig. 17f) [139]. Table 3 shows the performance of flexible paper-based electrochemical immunosensors based on 2D materials so far reported.

Cortisol plays a central role in managing many physiological processes in human physiology, including carbohydrate and amino acid metabolism, glucose optimization, and blood pressure control [140, 141]. Kinnamon et al. have developed a portable biological sensor for tracking the usual stress biomarker, cortisol, given in Fig. 18. The specificity of this sensor was attained by an affinity assay that uses nanosheets of $\mathrm{MoS}_{2}$ with the cortisol antibody as a sensing device. The sensing was performed by electrochemical impedance spectroscopy by measuring cortisol binding changes in $\mathrm{MoS}_{2}$ interfaces. Impedance responses to cyclic bending have been tested to determine the effect of mechanical stress, showing a minimum signal loss concerning relevant stress. This is the first time such a portable cortisol-monitoring biosensor was reported using non-faradaic impedance spectroscopy with high specificity and sensitivity $\left(1 \mathrm{ng} \mathrm{mL}^{-1}\right)$ [142].

More recently, Rebeca et al. fabricated a highly sensitive, selective, and powerful cortisol, versatile sensor array, leveraging the amazing performance of laser-induced graphene. The diurnal cycle of cortisol and a complex sweat stress reaction profile was investigated for the first time via an integrated sensing device. During sweat analyses, this sensor showed sensitivity around $1 \mathrm{ng} / \mathrm{mL}$ with a detection limit of around $0.08 \mathrm{ng} / \mathrm{mL}$ and was also capable of being close to real-time sweat analysis of cortisol at the nanogram per milliliter level. This lightweight, micro-fluidic patch is mechanically stable and can be laminated in conformity with the skin [143].

\section{Powering wearable/flexible sensors based on two-dimensional materials}

One of the significant challenges faced by wearable/flexible biosensors is finding an appropriate power source. The biosensor power requirement comes from three sources, firstly for identifying biomarkers, secondly for complex data processing, and finally, for the operation of wireless signal transceivers and multiple sensors [144, 145]. To address these power needs, multiple methods, such as the development of wearable energy sources, energy-efficient sensing devices, and adaptive algorithms, are required [145, 146].

Advances in wearable energy systems could not cope with the pace of advancement in wearable sensor technology. A common route block for efficient adaptation of wearable sensors is the limitation of inadequate wearable energy sources. Wearable power sources near the wearable electronic device should therefore be produced to eliminate the need for long 
Table 3 Overview of paper-based electrochemical immunosensors for clinical analysis

\begin{tabular}{|c|c|c|c|c|}
\hline Electrode & Analyte & Linear range & Limit of detection & References \\
\hline $\begin{array}{l}\text { anti-AFP/chitosan/r-GO } \\
\text { anti-CA125/chitosan/r-GO }\end{array}$ & $\begin{array}{l}\text { AFP } \\
\text { CA125 }\end{array}$ & $\begin{array}{l}0.001-100 \mathrm{ng} \mathrm{mL}^{-1} \\
0.001-100 \mathrm{ng} \mathrm{mL}^{-1}\end{array}$ & $\begin{array}{l}0.001 \mathrm{ng} \mathrm{mL}^{-1} \\
0.001 \mathrm{ng} \mathrm{mL}^{-1}\end{array}$ & \multirow[t]{3}{*}[122]{} \\
\hline anti-CA153/chitosan/r-GO & CA153 & $0.005-100 \mathrm{ng} \mathrm{mL}^{-1}$ & $0.005 \mathrm{ng} \mathrm{mL}^{-1}$ & \\
\hline anti-CEA/chitosan/r-GO & CEA & $0.005-100 \mathrm{ng} \mathrm{mL}^{-1}$ & $0.005 \mathrm{ng} \mathrm{mL}^{-1}$ & \\
\hline$\left(\mathrm{NH}_{2}-\mathrm{G}\right) /$ thionine (Thi)/Au NPs & $\begin{array}{l}\text { CEA standard } \\
\text { Clinical serum samples }\end{array}$ & $\begin{array}{l}50 \mathrm{pg} \mathrm{mL}^{-1}-500 \mathrm{ng} \mathrm{mL}^{-1} \\
1.75-64.20 \mathrm{ng} \mathrm{mL}^{-1}\end{array}$ & $\begin{array}{l}10 \mathrm{pg} \mathrm{mL}^{-1} \\
1.75 \mathrm{ng} \mathrm{mL}^{-1}\end{array}$ & {$[127]$} \\
\hline $\mathrm{rGO} / \mathrm{Thi} / \mathrm{Au} \mathrm{NPs}$ & CA125 & $0.1-200 \mathrm{U} \mathrm{mL}^{-1}$ & $0.01 \mathrm{U} \mathrm{mL}^{-1}$ & {$[128]$} \\
\hline BSA/ANTI-FTH/GO/SPGE & Ferritin & $1-1000 \mathrm{ng} \mathrm{mL}^{-1}$ & $0.19 \mathrm{ng} \mathrm{mL}^{-1}$ & {$[136]$} \\
\hline PANI/graphene & IFN- $\gamma$ & $5-1000 \mathrm{pg} \mathrm{mL} L^{-1}$ & $3.4 \mathrm{pg} \mathrm{mL}^{-1}$ & [129] \\
\hline $\mathrm{rGO} / \mathrm{Au} \mathrm{NPs}$ & E. coli & $1.5 \times 10^{2}-1.5 \times 10^{7} \mathrm{cfu} \mathrm{mL}^{-1}$ & $1.5 \times 10^{2} \mathrm{cfu} \mathrm{mL} \mathrm{m}^{-1}$ & [79] \\
\hline TR-GO & Anti-IgG & $0.3-7 \mu \mathrm{g} \mathrm{mL}^{-1}$ & $10 \mu \mathrm{g} \mathrm{mL}^{-1}$ & [139] \\
\hline
\end{tabular}

cables and allow for complete system integration with the body [147]. In this respect, researchers have explored many ways of developing wearable power devices, such as wearable batteries, wearable energy storage devices (supercapacitors [148] and Li-ion batteries [149]), energy conversion devices (piezoelectric/triboelectric nanogenerators [150] which harvest energy from human body motions, thermoelectric supercapacitors [151], solar cells [152], biofuel cells (BFC) [153]), hybrid power supplies which combine both energy conversion and energy storage devices together, and wireless energy transfer systems like RF antennas [147]. Choosing the best energy-harvesting option depends on how the sensor is
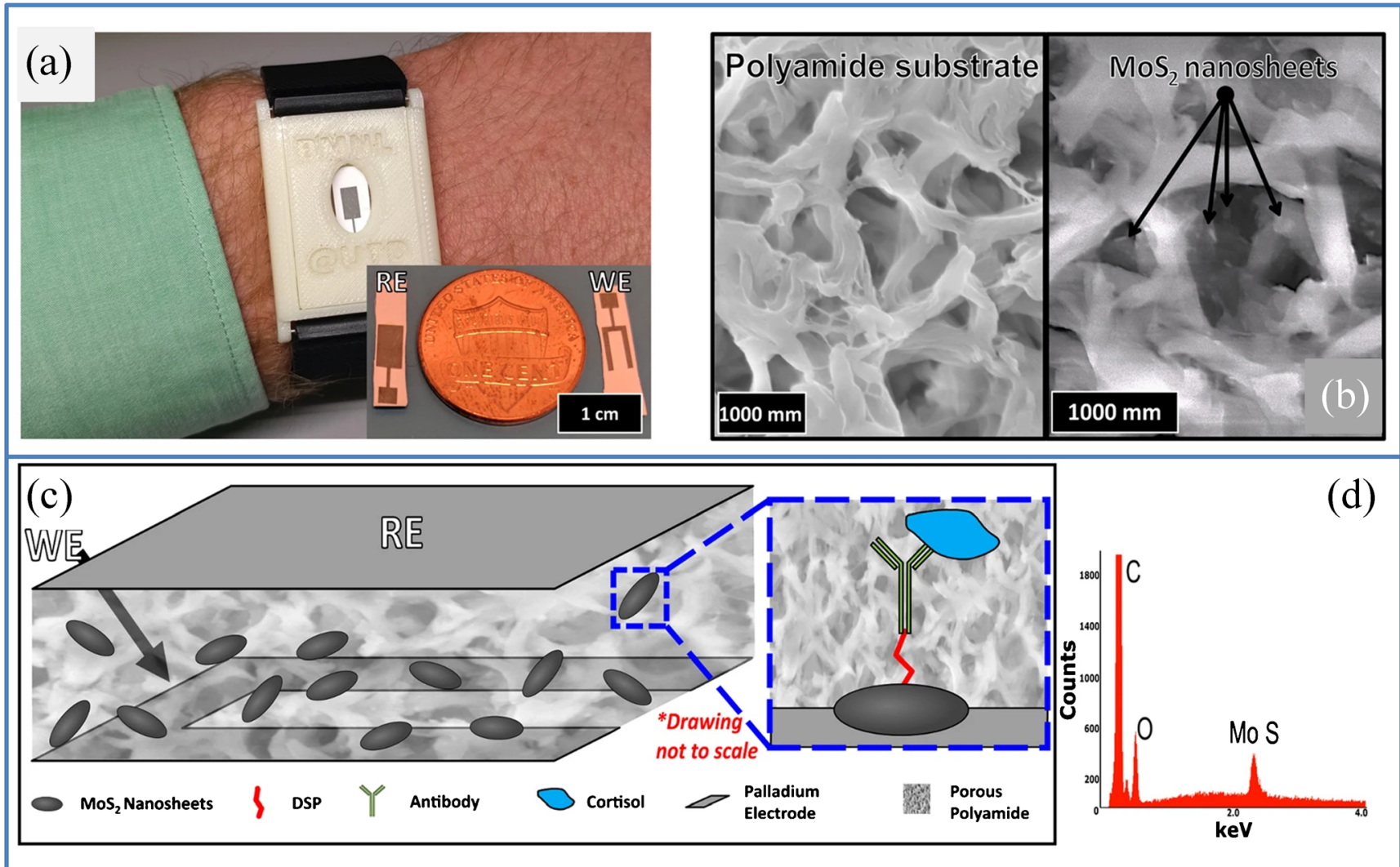

Fig. 18 a Illustration for the presumed cortisol transdermal sweat monitoring prototype device. b SEM images of polyamide substrate before and after $\mathrm{MoS}_{2}$ coating. c Schematic representation of the prepared $\mathrm{MoS}_{2}$-based cortisol sensor d EDAX spectra. Source: Kinnamon, David, Ghanta, Ramesh Lin, Kai Chun Muthukumar,
Sriram Prasad, Shalini- Portable biosensor for monitoring cortisol in low-volume perspired human sweat, Scientific Reports,7(2017), 113,https://doi.org/10.1038/s41598-017-13684-7, http:// creativecommons.org/licenses/by/4.0/ 
going to be used or worn. Alternative energy-harvesting approaches - which eliminate the need to modify or recharge a battery - have gained significant recent attention though batteries remain the most typical power sources for wearable devices. Self-powered energy harvesters based on biofuel cells, thermoelectric generator, solar cells, and triboelectric and piezoelectric nanogenerators have recently attracted great attention [150, 153].

The advantages of self-powered biosensor over batteries are compact size, low cost, light weight, limitless endurance, and environment-friendliness. Several studies have already reviewed self-powered biosensors for medical applications. Among these, Fu et al. and Chen et al. reviewed the advancement in the biofuel cell-based self-powered electrochemical biosensors and their practical application in biochemical detection. Choi et al. reported a self-powered BFC-based noninvasive glucose biosensor, which was integrated on a standard Band-Aid adhesive patch to detect glucose in human sweat. This biosensor was made up of graphene/chitosan/ GOx-based bioanaode and Ni/activate carbon (AC)-based cathode and polystyrene sulfonate:poly(3,4ethylenedioxythiophene) (PSS:PEDOT) as conductive reservoir. This system, which can be connected to the skin, can directly absorb sweat through capillary forces into a conductive reservoir that transforms chemical energy into electrical energy and measures glucose without any external power source [154]. Many teams are currently working on the problems for low-power wearable electrochemical devices of the next decade.

\section{Conclusion and future perspective}

This paper offers a brief analysis of some of the leading works on flexible/wearable electrical biosensors based on twodimensional materials. These electrochemical sensors based on two-dimensional materials demonstrate excellent prospects of biosensing in the field of healthcare. For these purposes, various types of flexible/wearable sensors manufactured on different substrates, such as polymer, textile, and paper with suitable reagents, modified with appropriate two-dimensional materials, either in the presence or absence of specific bioreceptors, have been suggested. The resulting devices take advantage of electrochemical detection and exploit the fascinating features that two-dimensional materials have, such as high surface area, biocompatibility, high selectivity, and electrocatalytic properties. Using these characteristics, the sensors mentioned above have been used to determine a broad range of biomolecules, including glucose, lactate, ascorbic acid, $\mathrm{H}_{2} \mathrm{O}_{2}$, and cancer-relevant biomarker proteomics. Remarkable achievements in paper-based electrochemical sensors based on two-dimensional materials include the development of electrochemical PADs for clinical analysis, which exhibit outstanding characteristics in terms of low cost, disposability, reliability, easier monitoring of complicated matrix such as blood, and eco-friendless. Graphene remains one of the most frequently used two-dimensional material for general sensory purposes and also serves as a substrate suitable for coupling with other chemical or biochemical substances to increase the sensor's selectivity; the other two-dimensional materials discussed in this paper have demonstrated better properties such as enhanced LOD and sensitivity. In the past few years, graphene with inorganic materials has become very popular. These inorganic hybrid materials incur an additional electrochemical catalytic capacity as well as functionalization ability [155]. In Tables 1, 2, and 3 are shown several reports of graphene with metal nanoparticles that have been published to date. These flexible/wearable electrochemical sensors made of graphene-doped metal nanoparticle increase the rate of transmission of electrons between the centers of enzyme activity and the electrode, thus improving the sensitivity of the sensor [43]. An efficient way to amplify electrochemical signals was also demonstrated by the decoration of TMD with these metal nanoparticles, which is evident from the literature review. Another major thing which we learn from the study of literature is that real specimens, and in particular biological samples, including blood, are still not well studied and most real specimens are artificial laboratory samples such as human serum samples. So the development of flexible/wearable electrochemical sensors with application in bio-analytical samples could be an interesting area to explore.

Though considerable effort has been made, the use of twodimensional nanosheets for electrochemical biosensing applications poses many challenges. A cost-effective yet tunable material synthesis process is a crucial issue for any twodimensional electrochemical biosensor, especially in competition with available technology. The second challenge is that most of the studies focus on graphene and $\mathrm{MoS}_{2}$, while other two-dimensional nanomaterials have been neglected, although this may indicate the possibility of using them as sensor platforms. Thirdly, it is essential to investigate the mechanisms of electron transfer between two-dimensional nanomaterials and biomaterials thoroughly. Improved understanding of how two-dimensional materials interact with different substances can provide more excellent opportunities for exploring sensor applications outside current target libraries and increasing sensitivity far beyond the actual detection threshold. The next challenge is to achieve desirable mechanical stability, which is a peculiar feature of the flexible and wearable sensor. Also, two-dimensional materials must be thoroughly assessed for their biocompatibility, environmental stability, and long-term toxicity. The next issue, biofouling, has been resolved to some degree by changing the electrode with a polymer coating or by certain chemical amendments. But it is complicated to achieve such a mechanically stable interference-preventing and biofouling layer. The risk of 
detecting extremely low rates of a biomolecule is also a severe problem in developing a suitable wearable bio affinity sensor. Another big problem is charging the wearable sensors. In addition to the impressive progress made in the manufacture of different electrochemical biosensors, more work and innovations are required to create a robust and fully automated biosensor based on two-dimensional materials. The development of user-free and calibration-free wearable electrochemical sensors and storage and stability issues should also be considered. However, additional efforts are underway to expand methodologies and functions, and to reach the next goals in these fields of analysis, and some remaining problems need to be addressed.

Acknowledgments The authors would like to acknowledge the financial support from the Department of Science and Technology (DST)-SERB Early Career Research project (Grant No. ECR/2017/001850), DSTNanomission (DST/NM/NT/2019/205(G)), DST-SHRI (DST/TDT/ SHRI-34/2018), Karnataka Science and Technology Promotion Society (KSTePS/VGST-RGS-F/2018-19/GRD NO. 829/315), startup grant, Jain University (11 (39)/17/013/2017SG), and Nanomission (SR/NM/NS-20/ 2014) for the characterization facilities. BC thanks Nandini Garg, Dr. T. Sakuntala, Dr. S.M. Yusuf, and Dr. A.K. Mohanty for the support and encouragement.

\section{Compliance with ethical standards}

Conflict of interest The authors declare that they have no conflict of interest.

\section{References}

1. Mardonova M, Choi Y. Review of wearable device technology and its applications to the mining industry. Energies. 2018;11(3): 547. https://doi.org/10.3390/en11030547.

2. Liu Y, Pharr M, Salvatore GA. Lab-on-skin: a review of flexible and stretchable electronics for wearable health monitoring. ACS Nano. 2017;11(10):9614-35. https://doi.org/10.1021/acsnano. $7 \mathrm{~b} 04898$.

3. Li Y, Fu J, Zhong C, Wu T, Chen Z, Hu W, Amine K, Lu J. Recent Advances inFlexible Zinc-Based Rechargeable Batteries. Adv Energy Mater. 2019;9:1-9. https://doi.org/10.1002/aenm. 201802605.

4. Tricoli A, Nasiri N, De S. Wearable and Miniaturized Sensor Technologies forPersonalized and Preventive Medicine. Adv Funct Mater. 2017;27:1-19. https://doi.org/10.1002/adfm. 201605271.

5. Jo J, Yoon J, Lee T, Cho HY, Lee JY, Choi JW. H2O2 biosensor consisted of hemoglobin-DNA conjugate on nanoporous gold thin film electrode with electrochemical signal enhancement. Nano Converg. 2019;6:1-8. https://doi.org/10.1186/s40580-018-0172$\mathrm{z}$.

6. Zhang P, Zhao X, Ji Y, Ouyang Z, Wen X, Li J, et al. Electrospinning graphene quantum dots into a nanofibrous membrane for dual-purpose fluorescent and electrochemical biosensors. J Mater Chem B. 2015;3(12):2487-96. https://doi.org/10. 1039/c4tb02092h.
7. Guo X. Surface plasmon resonance based biosensor technique: a review. J Biophotonics. 2012;5(7):483-501. https://doi.org/10. 1002/jbio.201200015.

8. Araque E, Villalonga R, Gamella M, Martínez-Ruiz P, Reviejo J, Pingarrón JM. Crumpled reduced graphene oxidepolyamidoamine dendrimer hybrid nanoparticles for the preparation of an electrochemical biosensor. J Mater Chem B. 2013;1: 2289-96. https://doi.org/10.1039/c3tb20078g.

9. Yang Y, Gao W. Wearable and flexible electronics for continuous molecular monitoring. Chem Soc Rev. 2019;48(6):1465-91.

10. Pang Y, Wang C, Lu LC, Wang C, Sun Z, Xiao R. Dual-SERS biosensor for one-step detection of microRNAs in exosome and residual plasma of blood samples for diagnosing pancreatic cancer. Biosens Bioelectron. 2019;130:204-13. https://doi.org/10. 1016/j.bios.2019.01.039.

11. Chen C, Xie Q, Yang D, Xiao H, Fu Y, Tan Y, et al. Recent advances in electrochemical glucose biosensors: a review. RSC Adv. 2013;3:4473-91. https://doi.org/10.1039/c2ra22351a.

12. Soldatkina OV, Soldatkin OO, Kasap BO, Kucherenko DY, Kucherenko IS, Kurc BA, Dzyadevych SV. A Novel Amperometric Glutamate Biosensor Based on Glutamate Oxidase Adsorbed on Silicalite. Nanoscale Res Lett. 2017;12(1): 260. https://doi.org/10.1186/s11671-017-2026-8.

13. Park J, Lee W, Kim I, Kim M, Jo S, Kim W, et al. Ultrasensitive detection of fibrinogen using erythrocyte membrane-draped electrochemical impedance biosensor. Sensors Actuators B Chem. 2019;293:296-303. https://doi.org/10.1016/j.snb.2019.05.016.

14. Gao H, Wen L, Tian J, Wu Y, Liu F, Lin Y, et al. A portable electrochemical immunosensor for highly sensitive point-of-care testing of genetically modified crops. Biosens Bioelectron. 2019;142:111504. https://doi.org/10.1016/j.bios.2019.111504.

15. Weng B, Morrin A, Shepherd R, Crowley K, Killard AJ, Innis PC, et al. Wholly printed polypyrrole nanoparticle-based biosensors on flexible substrate. J Mater Chem B. 2014;2:793-9. https://doi. org/10.1039/c3tb21378a.

16. Park YM, Lim SY, Jeong SW, Song Y, Bae NH, Hong SB, Choi BG, Lee SJ, Lee KG. Flexible nanopillar-based electrochemical sensors for genetic detection of foodborne pathogens. Nano Converg. 2018;5(1):15. https://doi.org/10.1186/s40580-0180147-0.

17. Noviana E, McCord CP, Clark KM, Jang I, Henry CS, Electrochemical paper-based devices: sensing approaches and progress toward practical applications. Lab Chip. 2020;20:9-34. https://doi.org/10.1039/c9lc00903e.

18. Jeerapan I, Poorahong S. Review-flexible and stretchable electrochemical sensing systems: materials, energy sources, and integrations. J Electrochem Soc. 2020;167:037573. https://doi.org/10. 1149/1945-7111/ab7117.

19. Zhu C, Du D, Lin Y. Graphene and graphene-like 2D materials for optical biosensing and bioimaging: a review. 2D Mater. 2015;2(3):32004. https://doi.org/10.1088/2053-1583/2/3/032004.

20. Bolotsky A, Butler D, Dong C, Gerace K, Glavin NR, Muratore C, et al. Two-dimensional materials in biosensing and healthcare: from in vitro diagnostics to optogenetics and beyond. ACS Nano. 2019;13:9781-810. https://doi.org/10.1021/acsnano. $9 b 03632$.

21. Akada K, Terasawa TO, Imamura G, Obata S, Saiki K. Control of work function of graphene by plasma assisted nitrogen doping. Appl Phys Lett. 2014;104(13):131602. https://doi.org/10.1063/1. 487042.

22. Shao Y, Wang J, Wu H, Liu J, Aksay IA, Lin Y. Graphene based electrochemical sensors and biosensors: a review. Electroanalysis. 2010;22:1027-36. https://doi.org/10.1002/elan.200900571.

23. Kalantar-Zadeh K, Ou JZ. Biosensors based on two-dimensional MoS2. ACS Sensors. 2016;1:5-16. https://doi.org/10.1021/ acssensors.5b00142. 
24. Wang QH, Kalantar-Zadeh K, Kis A, Coleman JN, Strano MS. Electronics and optoelectronics of two-dimensional transition metal dichalcogenides. Nat Nanotechnol. 2012;7:699-712. https://doi.org/10.1038/nnano.2012.193.

25. Cai Y, Zhang G, Zhang YW. Layer-dependent band alignment and work function of few-layer phosphorene. Sci Rep. 2014;4: 1-6. https://doi.org/10.1038/srep06677.

26. Chandra S, Chandra S. Wearable sensors for physiological parameters measurement: physics, characteristics, design and applications. 2017.

27. Rosen ED, Spiegelman BM. Adipocytes as regulators of energy balance and glucose homeostasis. Nature. 2006;444:847-53. https://doi.org/10.1038/nature05483.

28. Of D, Mellitus D. Diagnosis and classification of diabetes mellitus. Diabetes Care. 2014;37:81-90. https://doi.org/10.2337/ dc14-S081.

29. Yoo EH, Lee SY. Glucose biosensors: an overview of use in clinical practice. Sensors. 2010;10:4558-76. https://doi.org/10. 3390/s100504558.

30. Mokhtar SMA, Omar M, Bakar ZA, Yusof Y, Rizman ZI. Graphene-based wearable electrochemical glucose biosensor: a review. Int J Eng Technol. 2018;7:250-3. https://doi.org/10. 14419/ijet.v7i3.14.16902.

31. Samal R, Sekhar C. Rout,Chapter 12 - Wearable and Flexible Sensors Based on 2D and Nanomaterials, Editor(s): Morgan Hywel, Chandra Sekhar Rout, Dattatray J. Late, In Woodhead Publishing Series in Electronic and Optical Materials, Fundamentals and Sensing Applications of 2D Materials, Woodhead Publishing. 2019;437-463. https://doi.org/10.1016/ B978-0-08-102577-2.00012-9.

32. Lee H, Hong YJ, Baik S, Hyeon T, Kim DH. Enzyme-based glucose sensor: from invasive to wearable device. Adv Healthc Mater. 2018;7:1-14. https://doi.org/10.1002/adhm.201701150.

33. Wang Y, Xu H, Zhang J, Li G. Electrochemical sensors for clinic analysis. Sensors. 2008;8:2043-81. https://doi.org/10.3390/ s8042043.

34. Toghill KE, Compton RG. Electrochemical non-enzymatic glucose sensors: A perspective and an evaluation. 2010;5:12461301.

35. Heller A, Feldman B. Electrochemical glucose sensors and their applications in diabetes management. Chem Rev. 2008;108: 2482-505. https://doi.org/10.1021/cr068069y.

36. Cass AEG, Davis G, Francis GD, Allen H, Hill O, Aston WJ, et al. Ferrocene-mediated enzyme electrode for amperometric determination of glucose. Anal Chem. 1984;56:667-71. https://doi.org/ 10.1021/ac00268a018.

37. Tsujimura S, Kojima S, Kano K, Ikeda T, Sato M, Sanada H, et al. Novel FAD-dependent glucose dehydrogenase for a dioxygeninsensitive glucose biosensor. Biosci Biotechnol Biochem. 2006;70:654-9. https://doi.org/10.1271/bbb.70.654.

38. Sapountzi E, Braiek M, Vocanson F, Jaffrezic-renault N, Lagarde F. Chemical Gold nanoparticles assembly on electrospun poly (vinyl alcohol )/poly ( ethyleneimine )/ glucose oxidase nanofibers for ultrasensitive electrochemical glucose biosensing. Sensors and Actuators B. 2017;238:392-401. https://doi.org/10.1016/j.snb. 2016.07.062.

39. Zhao A, Zhang Z, Zhang P, Xiao S, Wang L, Dong Y, et al. 3D nanoporous gold scaffold supported on graphene paper: freestanding and flexible electrode with high loading of ultrafine PtCo alloy nanoparticles for electrochemical glucose sensing. Anal Chim Acta. 2016;938:63-71. https://doi.org/10.1016/j.aca.2016.08.013.

40. He W, Sun Y, Xi J, Abdurhman AAM, Ren J, Duan H. Printing graphene-carbon nanotube-ionic liquid gel on graphene paper: towards flexible electrodes with efficient loading of PtAu alloy nanoparticles for electrochemical sensing of blood glucose. Anal
Chim Acta. 2016;903:61-8. https://doi.org/10.1016/j.aca.2015. 11.019 .

41. Lee H, Choi TK, Lee YB, Cho HR, Ghaffari R, Wang L, Choi HJ, Chung TD, Lu N, Hyeon T, Choi SH, Kim DH. A graphene-based electrochemical device with thermoresponsive microneedles for diabetes monitoring and therapy. Nat Nanotechnol. 2016;11: 566-72. https://doi.org/10.1038/nnano.2016.38.

42. Abellán-Llobregat A, Jeerapan I, Bandodkar A, Vidal L, Canals A, Wang J, Morallón E. A stretchable and screen-printed electrochemical sensor for glucose determination in human perspiration. Biosens Bioelectron. 2017;91:885-91.

43. Pu Z, Wang R, Wu J, Yu H, Xu K, Li D. A flexible electrochemical glucose sensor with composite nanostructured surface of the working electrode. Vol. 230, Sens Actuators, B: Chemical. Elsevier B.V.; 2016. 801-809 p.

44. El-Kady MF, Strong V, Dubin S, Kaner RB. Laser scribing of high-performance and flexible graphene-based electrochemical capacitors. Science (80- ). 2012;335:1326-30. https://doi.org/10. 1126/science.1216744.

45. Zhang Y, Li N, Xiang Y, Wang D, Zhang P, Wang Y, Lu S, Xu R, Zhao J. A flexible non-enzymatic glucose sensor based on copper nanoparticles anchored on laser-induced graphene. Carbon N Y. 2020;156:506-13.

46. Yoon H, Nah J, Kim H, Ko S, Sharifuzzaman M, Barman SC, et al. A chemically modified laser-induced porous graphene based flexible and ultrasensitive electrochemical biosensor for sweat glucose detection. Sens Actuators B Chem. 2020;311:127866. https:// doi.org/10.1016/j.snb.2020.127866.

47. Cao L, Han GC, Xiao H, Chen Z, Fang C. A novel 3D paper-based microfluidic electrochemical glucose biosensor based on rGOTEPA/PB sensitive film. Anal Chim Acta. 2020;1096:34-43.

48. Xuan X, Yoon HS, Park JY. A wearable electrochemical glucose sensor based on simple and low-cost fabrication supported micropatterned reduced graphene oxide nanocomposite electrode on flexible substrate. Biosens Bioelectron. 2018;109:75-82. https:// doi.org/10.1016/j.bios.2018.02.054

49. Wang Z, Dong S, Gui M, et al. Graphene paper supported MoS2 nanocrystals monolayer with $\mathrm{Cu}$ submicron-buds: Highperformance flexible platform for sensing in sweat. Analytical Biochemistry. 2018;543:82-9. https://doi.org/10.1016/j.ab.2017. 12.010.

50. Yoon J, Lee SN, Shin MK, Kim HW, Choi HK, Lee T, et al. Flexible electrochemical glucose biosensor based on GOx/gold/ MoS2/gold nanofilm on the polymer electrode. Biosens Bioelectron. 2019;140:111343. https://doi.org/10.1016/j.bios. 2019.111343.

51. Vishnu N, Sahatiya P, Kong CY, Badhulika S. Large area, one step synthesis of $\mathrm{NiSe}_{2}$ films on cellulose paper for glucose monitoring in bio-mimicking samples for clinical diagnostics. Nanotechnology. 2019;30(35):355502.

52. Liu S, Hui KS, Hui KN. Flower-like copper cobaltite nanosheets on graphite paper as high-performance supercapacitor electrodes and enzymeless glucose sensors. ACS Appl Mater Interfaces. 2016;8:3258-67. https://doi.org/10.1021/acsami.5b11001.

53. Uribarri J, Oh MS, Carroll HJ. D-Lactic acidosis. A review of clinical presentation, biochemical features, and pathophysiologic mechanisms. Medicine (Baltimore). 1998;77:73-82. https://doi. org/10.1097/00005792-199803000-00001.

54. Prodromidis MI, Karayannis MI. Enzyme based amperometric biosensors for food analysis. 2002.

55. Rathee K, Dhull V, Dhull R, Singh S. Biosensors based on electrochemical lactate detection: a comprehensive review. Biochem Biophys Reports. 2016;5:35-54. https://doi.org/10.1016/j.bbrep. 2015.11.010. 
56. Labroo P, Cui Y. Flexible graphene bio-nanosensor for lactate. Biosens Bioelectron. 2013;41:852-6. https://doi.org/10.1016/j. bios.2012.08.024.

57. Stoller MD, Park S, Yanwu Z, An J, Ruoff RS. Graphene-based ultracapacitors. Nano Lett. 2008;8:3498-502. https://doi.org/10. 1021/nl802558y.

58. Lin KC, Muthukumar S, Prasad S. Flex-GO (flexible graphene oxide) sensor for electrochemical monitoring lactate in lowvolume passive perspired human sweat. Talanta. 2020;214: 120810. https://doi.org/10.1016/j.talanta.2020.120810.

59. Lei Y, Zhao W, Zhang Y, Jiang Q, He JH, Baeumner AJ, et al. A MXene-based wearable biosensor system for high-performance in vitro perspiration analysis. Small. 2019;15:1-10. https://doi. org/10.1002/smll.201901190.

60. Su S, Chao J, Pan D, Wang L, Fan C. Electrochemical sensors using two-dimensional layered nanomaterials. Electroanalysis. 2015;27:1062-72. https://doi.org/10.1002/elan.201400655.

61. Pisoschi AM, Pop A, Serban AI, Fafaneata C. Electrochemical methods for ascorbic acid determination. Electrochim Acta. 2014;121:443-60. https://doi.org/10.1016/j.electacta.2013.12. 127.

62. Jackowska K, Krysinski P. New trends in the electrochemical sensing of dopamine. Anal Bioanal Chem. 2013;405:3753-71. https://doi.org/10.1007/s00216-012-6578-2.

63. Yang Y, Song Y, Bo X, Min J, Pak OS, Zhu L, et al. A laserengraved wearable sensor for sensitive detection of uric acid and tyrosine in sweat. Nat Biotechnol. 2020;38:217-24. https://oi. org/10.1038/s41587-019-0321-x.

64. Bravo R, Hsueh CC, Jaramillo A, Brajter-Toth A. Possibilities and limitations in miniaturized sensor design for uric acid. Analyst. 1998;123:1625-30. https://doi.org/10.1039/a802341g.

65. Shamkhalichenar H, Choi J-W. Review-non-enzymatic hydrogen peroxide electrochemical sensors based on reduced graphene oxide. J Electrochem Soc. 2020;167:037531. https://doi.org/10. 1149/1945-7111/ab644a.

66. Abdurhman AA, Zhang Y, Zhang G, Wang S. Hierarchical nanostructured noble metal/metal oxide/graphene-coated carbon fiber: in situ electrochemical synthesis and use as microelectrode for real-time molecular detection of cancer cells. Anal Bioanal Chem. 2015;407(26):8129-36. https://doi.org/10.1007/s00216015-8989-3.

67. Liu H, Weng L, Yang C. A review on nanomaterial-based electrochemical sensors for $\mathrm{H} 2 \mathrm{O} 2, \mathrm{H} 2 \mathrm{~S}$ and $\mathrm{NO}$ inside cells or released by cells. Microchim Acta. 2017;184:1267-83. https://doi. org/10.1007/s00604-017-2179-2.

68. Zhang R, Chen W. Recent advances in graphene-based nanomaterials for fabricating electrochemical hydrogen peroxide sensors. Biosens Bioelectron. 2017;89:249-68. https://doi.org/10. 1016/j.bios.2016.01.080.

69. Lee CW, Suh JM, Jang HW. Chemical sensors based on twodimensional (2D) materials for selective detection of ions and molecules in liquid. Front Chem. 2019;7:1-21. https://doi.org/ 10.3389/fchem.2019.00708.

70. Cai W, Lai J, Lai T, Xie H, Ye J. Controlled functionalization of flexible graphene fibers for the simultaneous determination of ascorbic acid, dopamine and uric acid. Sens Actuators B Chem. 2016;224:225-32. https://doi.org/10.1016/j.snb.2015.09.079.

71. Late DJ, Huang YK, Liu B, Acharya J, Shirodkar SN, Luo J, et al. Sensing behavior of atomically thin-layered MoS2 transistors. ACS Nano. 2013;7(6):4879-91. https://doi.org/10.1021/ nn400026u.

72. Sha R, Vishnu N, Badhulika S. MoS2 based ultra-low-cost, flexible, non-enzymatic and non-invasive electrochemical sensor for highly selective detection of uric acid in human urine samples. Sens Actuators B Chem. 2019;279:53-60. https://doi.org/10. 1016/j.snb.2018.09.106.
73. de Araujo WR, Frasson CMR, Ameku WA, Silva JR, Angnes L, Paixão TRLC. Single-step reagentless laser scribing fabrication of electrochemical paper-based analytical devices. Angew Chem. 2017;129(47):15309-13. https://doi.org/10.1002/ange. 201708527.

74. Nayak P, Kurra N, Xia C, Alshareef HN. Highly Efficient Laser Scribed Graphene Electrodes for On-Chip Electrochemical Sensing Applications. Adv Electron Mater. 2016;2:1600185. https://doi.org/10.1002/aelm.201600185.

75. Silva VAOP, Fernandes-Junior WS, Rocha DP, Stefano JS, Munoz RAA, Bonacin JA, Janegitz BC. 3D-printed reduced graphene oxide/polylactic acid electrodes: A new prototyped platform for sensing and biosensing applications. Biosensors and Bioelectronics. 2020;170:112684. https://doi.org/10.1016/j.bios. 2020.112684.

76. Zan X, Bai H, Wang C, Zhao F, Duan H. Graphene paper decorated with a 2D Array of dendritic platinum nanoparticles for ultrasensitive electrochemical detection of dopamine secreted by live cells. Chem Eur J. 2016;22:5204-10. https://doi.org/10.1002/ chem. 201504454.

77. Ng AMH, Kenry TLC, Low HY, Loh KP. Highly sensitive reduced graphene oxide microelectrode array sensor. Biosens Bioelectron. 2015;65:265-73. https://doi.org/10.1016/j.bios. 2014.10.048

78. Kıranşan KD, Topçu E. Free-standing and flexible $\mathrm{MoS}_{2} / \mathrm{rGO}$ paper electrode for amperometric detection of folic acid. Electroanalysis. 2018;30:810-8. https://doi.org/10.1002/elan. 201700778.

79. Wang Y, Ping J, Ye Z, Wu J, Ying Y. Impedimetric immunosensor based on gold nanoparticles modified graphene paper for label-free detection of Escherichia coli O157: H7. Biosens Bioelectron. 2013;49:492-8. https://doi.org/10.1016/j. bios.2013.05.061.

80. Xiao F, Li Y, Zan X, Liao K, Xu R, Duan H. Growth of metalmetal oxide nanostructures on freestanding graphene paper for flexible biosensors. Adv Funct Mater. 2012;22:2487-94. https:// doi.org/10.1002/adfm.201200191.

81. Song R-M, Li Z-H, Wei P-J, Zhao X-L, Chen C, Zhu Z-G. Flexible Hydrogen Peroxide Sensors Based on Platinum Modified Free-Standing Reduced Graphene Oxide Paper. Appl Sci. 2018;8:848. https://doi.org/10.3390/app8060848.

82. Xiao F, Song J, Gao H, Zan X, Xu R, Duan H. Coating graphene paper with 2D-assembly of electrocatalytic nanoparticles: a modular approach toward high-performance flexible electrodes. ACS Nano. 2012;6:100-10. https://doi.org/10.1021/nn202930m.

83. Dong $\mathrm{S}, \mathrm{Xi}$ J, $\mathrm{Wu} \mathrm{Y}$, Liu H, Fu C, $\mathrm{Liu} \mathrm{H}$, et al. High loading $\mathrm{MnO}_{2}$ nanowires on graphene paper: facile electrochemical synthesis and use as flexible electrode for tracking hydrogen peroxide secretion in live cells. Anal Chim Acta. 2015;853:200-6. https://doi.org/10. 1016/j.aca.2014.08.004

84. Sun Y, Fang Z, Wang C, Ariyawansha KRRM, Zhou A, Duan H. Sandwich-structured nanohybrid paper based on controllable growth of nanostructured $\mathrm{MnO}_{2}$ on ionic liquid functionalized graphene paper as a flexible supercapacitor electrode. Nanoscale. 2015;7:7790-801. https://doi.org/10.1039/c5nr00946d.

85. Sun Y, Zheng H, Wang C, Yang M, Zhou A, Duan H. Ultrasonicelectrodeposition of PtPd alloy nanoparticles on ionic liquidfunctionalized graphene paper: towards a flexible and versatile nanohybrid electrode. Nanoscale. 2016;8:1523-34. https://doi. org/10.1039/c5nr06912b.

86. Peng Y, Lin D, Justin Gooding J, Xue Y, Dai L. Flexible fibershaped non-enzymatic sensors with a graphene-metal heterostructure based on graphene fibres decorated with gold nanosheets. Carbon N Y. 2018;136:329-36. https://doi.org/10. 1016/j.carbon.2018.05.004. 
87. Dağcı Kıranşan K, Aksoy M, Topçu E. Flexible and freestanding catalase-Fe3O4/reduced graphene oxide paper: enzymatic hydrogen peroxide sensor applications. Mater Res Bull. 2018;106:5765. https://doi.org/10.1016/j.materresbull.2018.05.032.

88. Ling W, Hao Y, Wang H, Xu H, Huang X. A novel Cu-metalorganic framework with two-dimensional layered topology for electrochemical detection using flexible sensors. Nanotechnology. 2019;30(42):424002.

89. Manjakkal L, Dervin S, Dahiya R. Flexible potentiometric $\mathrm{pH}$ sensors for wearable systems. RSC Adv. 2020;10(15):8594-617. https://doi.org/10.1039/d0ra00016g.

90. Li G, Wen D. Wearable biochemical sensors for human health monitoring: sensing materials and manufacturing technologies. J Mater Chem B. 2020;8:3423-36. https://doi.org/10.1039/ c9tb02474c.

91. Melai B, Salvo P, Calisi N, Moni L, Bonini A, Paoletti C, et al. A graphene oxide $\mathrm{pH}$ sensor for wound monitoring. Proc Annu Int Conf IEEE Eng Med Biol Soc EMBS. 2016;2016:1898-901. https://doi.org/10.1109/EMBC.2016.7591092.

92. Ang PK, Chen W, Wee AT, Loh KP. Solution-gated epitaxial graphene as pH sensor. J Am Chem Soc. 2008;130(44):143923. https://doi.org/10.1021/ja805090z.

93. Lei N, Li P, Xue W, Xu J. Simple graphene chemiresistors as ph sensors: Fabrication and characterization. Measurement Science and Technology. 2011;22(10):107002. https://doi.org/10.1088/ 0957-0233/22/10/107002.

94. Janczak D, Peplowski A, Wroblewski G, Gorski L, Zwierkowska E, Jakubowska M. Investigations of Printed Flexible $\mathrm{pH}$ Sensing Materials Based on Graphene Platelets and Submicron RuO2Powders. Journal of Sensors. 2017;2017:1-6. https://doi. org $/ 10.1155 / 2017 / 2190429$.

95. Piacenti da Silva M, Fernandes J, de Figueiredo N, Congiu M, Mulato M, de Oliveira Graeff C. Melanin as an active layer in biosensors. AIP Advances. 2014;4(3):037120. https://doi.org/10. 1063/1.4869638.

96. Tehrani Z, Whelan SP, Mostert AB, Paulin JV, Ali MM, Ahmadi $\mathrm{ED}$, et al. Printable and flexible graphene $\{\mathrm{pH}\}$ sensors utilising thin film melanin for physiological applications. 2D Mater. 2020;7(2):24008. https://doi.org/10.1088/2053-1583/ab72d5.

97. Veeralingam S, Sahatiya P, Kadu A, Mattela V, Badhulika S. Direct, one-step growth of $\mathrm{NiSe}_{2}$ on cellulose paper: a low-cost, flexible, and wearable with smartphone enabled multifunctional sensing platform for customized noninvasive personal healthcare monitoring. ACS Appl Electron Mater. 2019;1:558-68. https:// doi.org/10.1021/acsaelm.9b00022.

98. Su W, Xu J, Ding X. An Electrochemical pH Sensor Based on the Amino-Functionalized Graphene and Polyaniline Composite Film. IEEE Transactions on NanoBioscience. 2016;15(8):812-9. https://doi.org/10.1109/tnb.2016.2625842.

99. Liao W, Wei W, Tong Y, Chim W, Zhu C. Low-Frequency Noise in Layered ReS2 Field Effect Transistors on HfO2 and Its Application for $\mathrm{pH}$ Sensing. ACS Applied Materials \& Interfaces. 2018;10(8):7248-55. https://doi.org/10.1021/acsami. 8 b00193.

100. Cristea C, Florea A, Tertiș M. Săndulescu R Immunosensors; London. Intech Open Limited. 2015. https://doi.org/10.5772/ 60524.

101. Holford TRJ, Davis F, Higson SPJ. Recent trends in antibody based sensors. Biosens Bioelectron. 2012;34:12-24. https://doi. org/10.1016/j.bios.2011.10.023.

102. Wujcik EK, Wei H, Zhang X, Guo J, Yan X, Sutrave N, et al. Antibody nanosensors: a detailed review. RSC Adv. 2014;4: 43725-45. https://doi.org/10.1039/c4ra07119k.

103. Gil EDS, Kubota LT, Yamamoto YI. Alguns aspectos de imunoensaios aplicados à química analítica. Quim Nova.
1999;22:874-81. https://doi.org/10.1590/s010040421999000600015 .

104. Killard AJ, Deasy B, O'Kennedy R, Smyth MR. Antibodies: production, functions and applications in biosensors. TrAC Trends in Analytical Chemistry. 1995;14:257-66. https://doi.org/10.1016/ 0165-9936(95)91618-3.

105. Omidfar K, Khorsand F, Darziani AM. New analytical applications of gold nanoparticles as label in antibody based sensors. Biosens Bioelectron. 2013;43:336-47. https://doi.org/10.1016/j. bios.2012.12.045.

106. Suri CR, Raje M, Varshney GC. Immunosensors for pesticide analysis: antibody production and sensor development. Crit Rev Biotechnol. 2002;22:15-32. https://doi.org/10.1080/ 07388550290789441.

107. Riccardi CS, Costa PIY. Amperometric immunosensor. Quim Nova. 2002;2(2) ISSN:1678-7064.

108. Mistry KK, Layek K, Mahapatra A, RoyChaudhuri C, Saha H. A review on amperometric-type immunosensors based on screenprinted electrodes. Analyst. 2014;139:2289-311. https://doi.org/ 10.1039/c3an02050a.

109. Pei X, Zhang B, Tang J, Liu B, Lai W, Tang D. Sandwich-type immunosensors and immunoassays exploiting nanostructure labels: a review. Anal Chim Acta. 2013;758:1-18. https://doi.org/ 10.1016/j.aca.2012.10.060.

110. Marco MP, Gee S, Hammock BD. Immunochemical techniques for environmental analysis I. Immunosensors Trends Anal Chem. 1995;14:341-50. https://doi.org/10.1016/0165-9936(95)97062-6.

111. Basu PK, Indukuri D, Keshavan S, Navratna V, Vanjari SRK, Raghavan S, et al. Graphene based E. coli sensor on flexible acetate sheet. Sens actuators. B Chem. 2014;190:342-7. https://doi. org/10.1016/j.snb.2013.08.080.

112. Jabari E, Toyserkani E. Micro-scale aerosol-jet printing of graphene interconnects. Carbon N Y. 2015;91:321-9. https:// doi.org/10.1016/j.carbon.2015.04.094.

113. Parate K, Pola CC, Rangnekar SV, Mendivelso-Perez DL, Smith EA, Hersam MC, Gomes CL, Claussen JC. Aerosol-jet-printed graphene electrochemical histamine sensors for food safety monitoring. 2D Mater. 2020;7(3):034002. https://doi.org/10.1088/ 2053-1583/ab8919.

114. Liu F, Choi KS, Park TJ, Lee SY, Seo TS. Graphene-based electrochemical biosensor for pathogenic virus detection. Biochip J. 2011;5:123-8. https://doi.org/10.1007/s13206-011-5204-2.

115. Parate K, Rangnekar SV, Jing D, Mendivelso-Perez DL, Ding S, Secor EB, et al. Aerosol-jet-printed graphene immunosensor for label-free cytokine monitoring in serum. ACS Appl Mater Interfaces. 2020;12:8592-603. https://doi.org/10.1021/acsami. 9b22183.

116. Han TH, Lee Y, Choi MR, Woo SH, Bae SH, Hong BH, Ahn JH, Lee TW. Extremely efficient flexible organic light-emitting diodes with modified graphene anode. Nat Photonics. 2012;6:105-10. https://doi.org/10.1038/nphoton.2011.318.

117. Park H, Chang S, Zhou X, Kong J, Palacios T, Gradečak S. Flexible graphene electrode-based organic photovoltaics with record-high efficiency. Nano Lett. 2014;14:5148-54. https://doi. org/10.1021/nl501981f.

118. Tian H, Shu Y, Cui YL, Mi WT, Yang Y, Xie D, Ren TL. Scalable fabrication of high-performance and flexible graphene strain sensors. Nanoscale. 2014;6:699-705. https://doi.org/10.1039/ c3nr04521h.

119. Yaqoob U, Phan DT, Uddin ASMI, Chung GS. Highly flexible room temperature NO2 sensor based on MWCNTs-WO3 nanoparticles hybrid on a PET substrate. Sens Actuators B Chem. 2015;221:760-8. https://doi.org/10.1016/j.snb.2015.06.137.

120. Embretson J, Zupancic M, Ribas JL, Burke A, Racz P, TennerRacz K, et al. Massive covert infection of helper T lymphocytes 
and macrophages by HIV during the incubation period of AIDS. Nature. 1993;362:359-62. https://doi.org/10.1038/362359a0.

121. Shin M, Yoon J, Yi C, Lee T, Choi JW. Flexible HIV-1 biosensor based on the au/MoS2 nanoparticles/au nanolayer on the PET substrate. Nanomaterials. 2019;9:1-12. https://doi.org/10.3390/ nano9081076.

122. Wu Y, Xue P, Kang Y, Hui KM. Paper-based microfluidic electrochemical immunodevice integrated with nanobioprobes onto graphene film for ultrasensitive multiplexed detection of cancer biomarkers. Anal Chem. 2013;85:8661-8. https://doi.org/10. 1021/ac401445a.

123. Wang R, Chen X, Ma J, Ma Z. Ultrasensitive detection of carcinoembryonic antigen by a simple label-free immunosensor. Sens Actuators B Chem. 2013;176:1044-50. https://doi.org/10. 1016/j.snb.2012.10.001.

124. Miao J, Wang X, Lu L, Zhu P, Mao C, Zhao H, et al. Electrochemical immunosensor based on hyperbranched structure for carcinoembryonic antigen detection. Biosens Bioelectron. 2014;58:9-16. https://doi.org/10.1016/j.bios.2014. 02.024 .

125. Hawkridge AM, Muddiman DC. Mass spectrometry-based biomarker discovery: toward a global proteome index of individuality. Annu Rev Anal Chem. 2009;2:265-77. https://doi.org/10. 1146/annurev.anchem.1.031207.112942.

126. Wang R, Di J, Ma J, Ma Z. Highly sensitive detection of cancer cells by electrochemical impedance spectroscopy. Electrochim Acta. 2012;61:179-84. https://doi.org/10.1016/j.electacta.2011. 11.112.

127. Wang Y, Xu H, Luo J, Liu J, Wang L, Fan Y, et al. A novel labelfree microfluidic paper-based immunosensor for highly sensitive electrochemical detection of carcinoembryonic antigen. Biosens Bioelectron. 2016;83:319-26. https://doi.org/10.1016/j.bios. 2016.04.062.

128. Fan Y, Shi S, Ma J, Guo Y. A paper-based electrochemical immunosensor with reduced graphene oxide/thionine/gold nanoparticles nanocomposites modification for the detection of cancer antigen 125. Biosens Bioelectron. 2019;135:1-7. https://doi.org/ 10.1016/j.bios.2019.03.063.

129. Ruecha N, Shin K, Chailapakul O, Rodthongkum N. Label-free paper-based electrochemical impedance immunosensor for human interferon gamma detection. Sens Actuators B Chem. 2019;279: 298-304. https://doi.org/10.1016/j.snb.2018.10.024.

130. Amreen K, Kumar AS. Electrochemical redox signaling of hemoglobin in human whole blood and its relevance to anemia and thalassemia diagnosis. Analyst. 2016;141:2145-9. https://doi. org/10.1039/c5an02646f.

131. Amayreh M, Hourani M. Direct electrochemical determination of hemoglobin in blood using iodine-coated platinum polycrystalline electrode. Anal Bioanal Chem Res. 2019;6:59-68. https://doi.org/ 10.22036/abcr.2018.125953.1198

132. Dutta P, Meher N, Malik AH, Choudhury B, Iyer PK. Polyfluorene-based bioconjugates for selective detection of ferritin in normal and cancer human blood serums. ACS Appl Polym Mater. 2019;1:18-26. https://doi.org/10.1021/acsapm.8b00016.

133. Dignass A, Farrag K, Stein J. Limitations of serum ferritin in diagnosing iron deficiency in inflammatory conditions. Int $\mathrm{J}$ Chronic Dis. 2018;2018:1-11. https://doi.org/10.1155/2018/ 9394060.

134. Yoon HC, Yang H, Yo BS. Ferritin immunosensing on microfabricated electrodes based on the integration of immunoprecipitation and electrochemical signaling reactions. Anal Sci. 2004;20:1249-53. https://doi.org/10.2116/analsci.20.1249.
135. Szunerits S, Mishyn V, Grabowska I, Boukherroub R. Electrochemical cardiovascular platforms: current state of the art and beyond. Biosens Bioelectron. 2019;131:287-98. https://doi. org/10.1016/j.bios.2019.02.010.

136. Boonkaew S, Teengam P, Jampasa S, Rengpipat S, Siangproh W, Chailapakul O. Cost-effective paper-based electrochemical immunosensor using a label-free assay forsensitive detection of ferritin. Analyst. 2020. https://doi.org/10.1039/d0an00564a.

137. Akhavan O, Ghaderi E, Esfandiar A. Wrapping bacteria by graphene nanosheets for isolation from environment, reactivation by sonication, and inactivation by near-infrared irradiation. J Phys Chem B. 2011;115:6279-88. https://doi.org/10.1021/jp200686k.

138. Dikin DA, Stankovich S, Zimney EJ, Piner RD, Dommett GHB, Evmenenko G, et al. Preparation and characterization of graphene oxide paper. Nature. 2007;448:457-60. https://doi.org/10.1038/ nature06016.

139. Loo AH, Bonanni A, Ambrosi A, Poh HL, Pumera M. Impedimetric immunoglobulin $\mathrm{G}$ immunosensor based on chemically modified graphenes. Nanoscale. 2012;4:921-5. https://doi. org/10.1039/c2nr11492e.

140. Sekar M, Sriramprabha R, Sekhar PK, Bhansali S, Ponpandian N, Pandiaraj M, et al. Review-towards wearable sensor platforms for the electrochemical detection of cortisol. J Electrochem Soc. 2020;167:067508. https://doi.org/10.1149/1945-7111/ab7e24.

141. Tuteja SK, Ormsby C, Neethirajan S. Noninvasive label-free detection of cortisol and lactate using graphene embedded screenprinted electrode. Nano-Micro Lett. 2018;10:1-10. https://doi.org/ 10.1007/s40820-018-0193-5.

142. Kinnamon D, Ghanta R, Lin KC, Muthukumar S, Prasad S. Portable biosensor for monitoring cortisol in low-volume perspired human sweat. Sci Rep. 2017;7:1-13. https://doi.org/10. 1038/s41598-017-13684-7.

143. Torrente-Rodríguez RM, Tu J, Yang Y, Min J, Wang M, Song Y, et al. Investigation of cortisol dynamics in human sweat using a graphene-based wireless mHealth system. Matter. 2020;2:921-37. https://doi.org/10.1016/j.matt.2020.01.021.

144. Ha M, Lim S, Ko H. Wearable and flexible sensors for userinteractive health-monitoring devices. J Mater Chem B. 2018;6: 4043-64. https://doi.org/10.1039/c8tb01063c.

145. Bandodkar AJ, Wang J. Non-invasive wearable electrochemical sensors: a review. Trends Biotechnol. 2014;32:363-71. https:// doi.org/10.1016/J.TIBTECH.2014.04.005.

146. Kim J, Campbell AS, de Ávila BEF, Wang J. Wearable biosensors for healthcare monitoring. Nat Biotechnol. 2019;37:389-406. https://doi.org/10.1038/s41587-019-0045-y.

147. Yáñez-Sedeño P, Campuzano S, Pingarrón JM. Screen-printed electrodes: promising paper and wearable transducers for (bio)sensing. Biosensors. 2020;10:76. https://doi.org/10.3390/ BIOS10070076.

148. Zhang Z, Wang P, Miao X, Zhang P, Yin L. Flexible and wearable lithium-ion batteries. Flex Wearable Electron Smart Cloth. 2020;131-62. https://doi.org/10.1002/9783527818556.ch6.

149. Zhang H, Cao Y, Chee MOL, Dong P, Ye M, Shen J. Recent advances in micro-supercapacitors. Nanoscale. 2019;11:580721. https://doi.org/10.1039/C9NR01090D.

150. Zhao L, Li H, Meng J, Li Z. The recent advances in self-powered medical information sensors. InfoMat. 2020;2:212-34. https://doi. org/10.1002/inf2.12064.

151. Deng F, Qiu H, Chen J, Wang L, Wang B. Wearable thermoelectric power generators combined with flexible supercapacitor for low-power human diagnosis devices. IEEE Trans Ind Electron. 2017;64:1477-85. https://doi.org/10.1109/TIE.2016.2613063. 
152. Hashemi SA, Ramakrishna S, Aberle AG. Recent progress in flexible-wearable solar cells for self-powered electronic devices. Energy Environ Sci. 2020;13:685-743. https://doi.org/10.1039/ c9ee03046h.

153. Fu L, Liu J, Hu Z, Zhou M. Recent advances in the construction of biofuel cells based self-powered electrochemical biosensors: a review. Electroanalysis. 2018;30:2535-50. https://doi.org/10.1002/ elan.201800487.
154. Cho E, Mohammadifar M, Choi S. A single-use, self-powered, paper-basedsensor patch for detection of exercise-induced hypoglycemia. Micromachines. 2017;8:265. https://doi.org/10.3390/ mi8090265.

155. Fang Y, Wang E. Electrochemical biosensors on platforms of graphene. Chem Commun. 2013;49:9526-39. https://doi.org/10. 1039/c3cc44735a.

Publisher's note Springer Nature remains neutral with regard to jurisdictional claims in published maps and institutional affiliations. 